\title{
Computational modeling and sub-grid scale stabilization of incompressibility and convection in the numerical simulation of friction stir welding processes
}

\author{
C. Agelet de Saracibar ${ }^{\mathrm{a}, \mathrm{b}}$, M. Chiumenti ${ }^{\mathrm{a}, \mathrm{b}}$, M. Cervera ${ }^{\mathrm{a}, \mathrm{b}}$, \\ N. Dialami ${ }^{\mathrm{b}}$, A. Seret ${ }^{\mathrm{b}, \mathrm{c}}$ \\ ${ }^{a}$ ETS Ingenieros de Caminos, Canales y Puertos, Universidad Politécnica de Cataluña (UPC), Edificio C1, Campus \\ Norte, UPC, Jordi Girona 1-3, 08034, Barcelona, Spain \\ ${ }^{\mathrm{b}}$ International Center for Numerical Methods in Engineering (CIMNE), Gran Capitán s/n, 08034 Barcelona, Spain \\ ${ }^{\mathrm{c}}$ Ecole Nationale Supérieure des Mines de Saint-Étienne, 158 cours Fauriel, 42023 St-Étienne, Cedex 2, France \\ agelet@cimne.upc.edu, michele@cimne.upc.edu, miguel.cervera@upc.edu, \\ narges@,cimne.upc.edu, anthony.seret@mines-saint-etienne.org
}

May 28, 2013

Submitted to

Archives of Computational Methods in Engineering 


\title{
Computational modeling and sub-grid scale stabilization of incompressibility and convection in the numerical simulation of friction stir welding processes
}

\author{
C. Agelet de Saracibar ${ }^{\mathrm{a}, \mathrm{b}}$, M. Chiumenti, ${ }^{\mathrm{a}, \mathrm{b}}$, M. Cervera ${ }^{\mathrm{a}, \mathrm{b}}$, \\ N. Dialami ${ }^{\mathrm{b}}$ A. Seret ${ }^{\mathrm{b}, \mathrm{c}}$ \\ ${ }^{a}$ ETS Ingenieros de Caminos, Canales y Puertos, Universidad Politécnica de Cataluña (UPC), Edificio C1, Campus \\ Norte, UPC, Jordi Girona 1-3, 08034, Barcelona, Spain \\ ${ }^{\mathrm{b}}$ International Center for Numerical Methods in Engineering (CIMNE), Gran Capitán s/n, 08034 Barcelona, Spain \\ ${ }^{\mathrm{c}}$ Ecole Nationale Supérieure des Mines de Saint-Étienne, 158 cours Fauriel, 42023 St-Étienne, Cedex 2, France
}

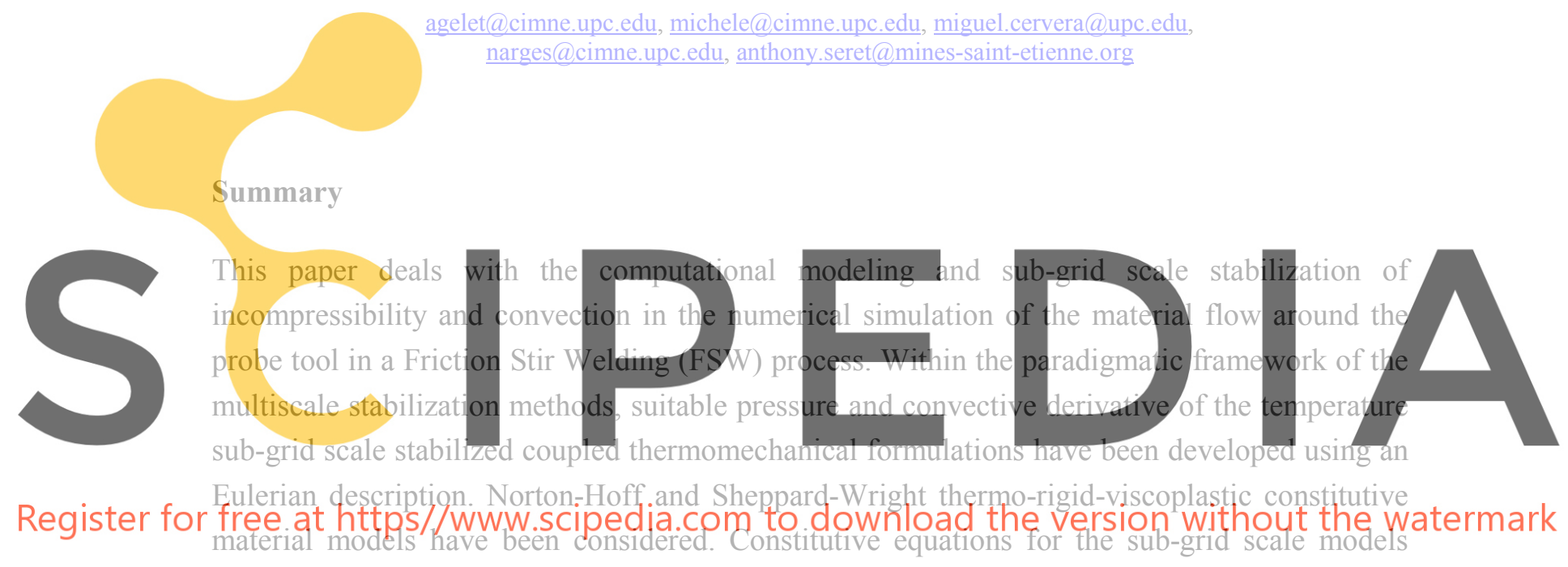

have been proposed and an approximation of the sub-grid scale variables has been given. In

particular, Algebraic Sub-grid Scale (ASGS) and Orthogonal Sub-grid Scale (OSGS) methods for mixed velocity, pressure and temperature P1/P1/P1 linear elements have been considered. Furthermore, it has been shown that well known classical stabilized formulations, such as the Galerkin Least-Squares (GLS) for incompressible (or quasi-incompressible) problems or the Streamline Upwind/Petrov-Galerkin (SUPG) method for convection dominant problems, can be recovered as particular cases of the multiscale stabilization framework considered. Using a product formula algorithm for the solution of the coupled thermomechanical problem, the resulting algebraic system of equations has been solved using a staggered procedure in which a mechanical problem, defined by the linear momentum balance equation, under quasi-static conditions, and the incompressibility equation, is solved first at constant temperature. Then a thermal problem, defined by the energy balance equation, is solved keeping constant the mechanical variables, i.e. velocity and pressure. The computational model has been implemented in an enhanced version of the finite element software COMET, developed by the authors at the International Center for Numerical Methods in Engineering (CIMNE). Two numerical examples have been considered. The first one deals with the numerical simulation of a coupled thermomechanical flow in a $2 \mathrm{D}$ rectangular domain. Steady-state and transient 
conditions have been considered. The goal of this numerical example has been the comparison between different sub-grid scale stabilization methods for the velocity and temperature equations. In particular, using a GLS stabilization method for the pressure equation, a comparison between SUPG and OSGS convective stabilization methods has been performed. Additionally, using a SUPG stabilization method for the temperature equation, a comparison between GLS and OSGS pressure stabilization methods has been done. The second example deals with the 3D numerical simulation of a representative FSW process. Numerical results obtained have been compared with experimental results available in the literature. A good agreement on the temperature distribution has been obtained and predicted peak temperatures compare well, both in value and position, with the experimental results available.

Version 6.1: May 28, 2013

Keywords: Variational multiscale methods, stabilized finite element methods, algebraic sub-grid scales, orthogonal sub-grid scales, stabilization of incompressibility and convection, thermally coupled rigid-plastic solids, friction stir welding

1 Introduction, motivation and goals
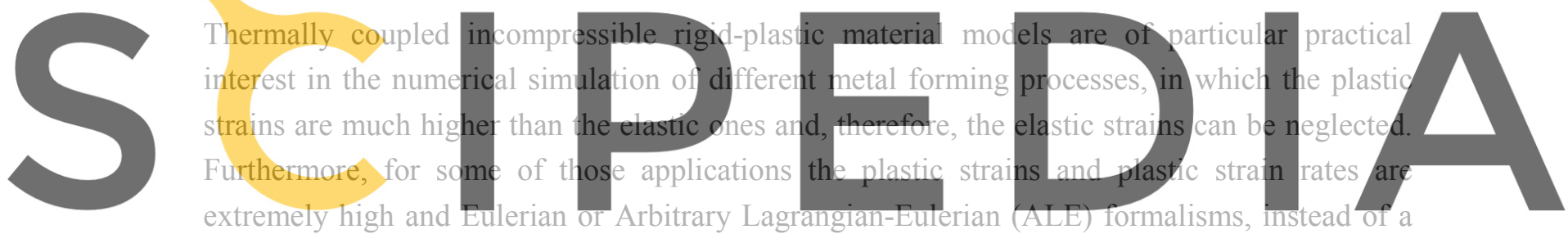

Lagrangian one, are far more convenient from the numerical point of view. Those situations free at https//w.wW.scipedia.com to downloa.d the version without the watermark arise, for instance, in the numerical simulation of Friction Stir Welding (FSW) processes $[6,7,8,33,34]$.

The formulation of thermally coupled incompressible rigid-plastic material models using Eulerian or ALE formalisms is of particular interest from the numerical point of view for different reasons. The need to stabilize the standard Galerkin finite element approximation comes from two main sources. Due to the incompressibility constraints, pressure instabilities appear if we wish to use equal velocity-pressure interpolations. On the other hand, Eulerian or ALE formalisms introduce convection terms in the governing equations and convection instabilities may arise for convection dominant problems. As it is now well known, both sources of instabilities can be overcome by using sub-grid scale finite element stabilized formulations $[57,58,59,60]$.

Sub-grid scale finite element stabilized methods have been widely used in Computational Fluid Dynamics (CFD) and they were initially developed by Hughes et al. (1986) for the Stokes problem [57].

Sub-grid scale models were first introduced by Hughes (1995) [58]. The Variational Multiscale (VMS) method - a paradigm for computational mechanics - was introduced by Hughes et al. (1998) [59]. See also Hughes et al. (2004) [60] for a presentation of multiscale and stabilized methods. The basic idea was to split the exact continuous unknowns into two 
components or scales: the finite-element component and the sub-grid scale or subscale component. The subscales represent the component of the exact continuous solution which cannot be captured by the finite element partition. The particular approximation used for these sub-grid scales defines the numerical model.

Within the CFD framework, VMS methods have been used by Codina (1998) [37] for the diffusion-convection reaction problem, Codina (2000) [39] to stabilize incompressibility and convection through orthogonal subscales, first introducing the Orthogonal Sub-grid Scale (OSGS) method, Codina (2001) [41] for generalized stationary incompressible flows, Codina (2002) [42] for transient incompressible flows using dynamic orthogonal subscales, Hughes et al. (2004) [60] for the incompressible Navier-Stokes equations, Codina et al. (2007) [43] for transient incompressible flows using dynamic subscales, Codina and Principe (2007) [44] for thermally coupled incompressible flows using nonlinear dynamic subscales, Principe (2008) [68], Principe and Codina (2008) [69], and Avila et al. (2010) [14] for the low Mach number flow equation, and Codina et al. (2010) [45] for turbulent thermally coupled incompressible flows.

Codina et al. (2007) [43] showed that it was worth to track the subscales in time in a variational multiscale approach to the transient incompressible Navier-Stokes equations and to take into account all their contributions in the convective term. They pointed out two main reasons. The first and very simple reason was that it leaded to global momentum conservation, a

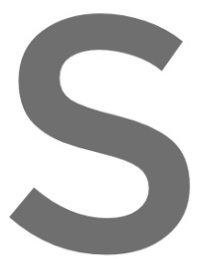
rare property. The sdcond one was the door opening
from the point of view of the time integration
resulting formulation leaded in a natural way
parameters with the time step, while steady-state the confliet about the d sign of the stabilization
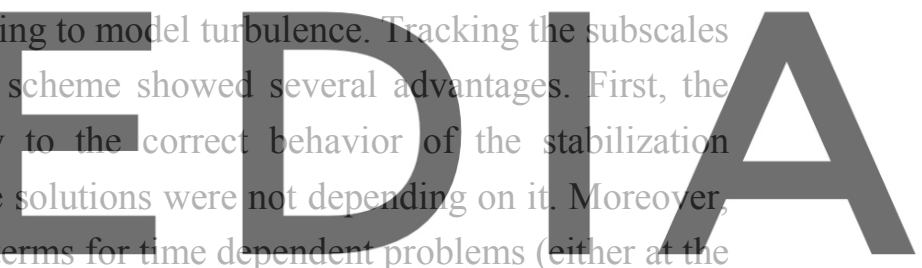

semi-discrete or the fully discrete level) disappeared, since space discretization (scale splitting)



and the improvement in accuracy with respect to quasi-static subscales was notorious

Codina and Principe (2007) [44] showed that the use of dynamic subscales had also several benefits in the numerical simulation of thermally coupled incompressible flows, such as improved time stability and accuracy, particularly when $\delta t \rightarrow 0$, correct behavior of the stabilization parameters with the time step size, commutation of space discretization (subscale splitting) and time discretization, and the coupling of velocity and temperature subscales was dealt with in a natural way. Numerical results confirmed the conclusions drawn for isothermal flows, yielding a more accurate formulation, in which oscillations originated by initial transients were eliminated and numerical dissipation was minimized.

Within the framework of Computational Solid Mechanics (CSM) sub-grid scale stabilization methods have been used by Chiumenti et al. (2002) [29] for incompressible linear elasticity, Cervera et al. (2003) [22], Christ et al. (2003) [35] and Chiumenti et al. (2004) [30] for incompressible linear elasticity and small strains J2 plasticity, Cervera et al. (2004) [23] for small strains J2 continuum damage models, Agelet de Saracibar et al. (2004) [4] for infinitesimal strains and finite deformation J2 plasticity, and Agelet de Saracibar et al. (2006) [5] for finite deformation J2 plasticity. They used sub-grid scale finite element stabilized mixed 
displacement/pressure formulations, in which the sub-grid scale pressure was neglected. Algebraic Sub-grid Scale (ASGS) and Orthogonal Sub-grid Scale (OSGS) methods were used.

Cervera et al. (2010) [25,26] used sub-grid scale finite element stabilized mixed stress/displacement and strain/displacement formulations for nonlinear (damage) solid mechanics models. Chiumenti et al. (2010) [32] used sub-grid scale finite element stabilized mixed strain/displacement and displacement/pressure formulations for Mode-I and Mode-II strain localization, using Rankine and J2 elasto-plasticity models, respectively. Sub-grid scale finite element stabilization methods, using continuum damage models and a mixed strain/displacement formulation, have been used by Cervera et al. $(2010,2011)$ [26,27] to address the problem of strain localization for tensile and mixed-mode cracking within the socalled smeared crack approach. Numerical examples showed that the resulting procedure was remarkably robust, it was not required the use of auxiliary tracking techniques and the results obtained were not suffering from spurious mesh bias dependence.

Chiumenti et al. (2010) [31] used a sub-grid scale finite element stabilized mixed displacement/pressure/temperature for the numerical simulation of Shaped Metal Deposition (SMD) processes.

Agelet de Saracibar et al. (2010) [6,7], Agelet de Saracibar et al. (2011) [8], Chiumenti et al. (2012) [33], and Dialami et al. (2012) [52] used a sub-grid scale finite element stabilized mixed velocity/pressure/ temperature formulation for coupled thermo-rigid-plastic models,

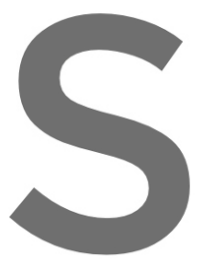
using Eulerian and Arbitraty Lagrangian Eulerion
simulation of Friction \$tir Wdding (FSW) prodes
quasi-static sub-grid scales, neglecting the sub-gr
component of the velocity in the convective term
Chiumenti et al. (2013)-347 used a novel


linear analysis with incompressibility constraints typically found in the numerical simulation of

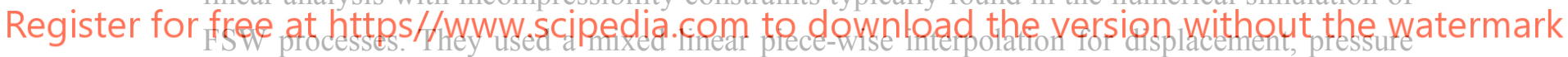

and stress fields, respectively, resulting in an enhanced stress field approximation which enables for stress accurate results in nonlinear computational mechanics.

Friction Stir Welding (FSW) is a new method of welding in solid state, created and patented by The Welding Institute (TWI) in 1991 [77]. In FSW a cylindrical, shouldered tool with a profiled probe is rotated and slowly plunged into the joint line between two pieces of sheet or plate material, which are butted together. The parts have to be clamped onto a backing bar in a manner that prevents the abutting joint faces from being forced apart. Once the probe has been completely inserted, it is moved with a small tilt angle in the welding direction. The shoulder applies a pressure on the material to constrain the plasticised material around the probe tool. Due to the advancing and rotating effect of the probe and shoulder of the tool along the seam, an advancing side and a retreating side are formed and the softened and heated material flows around the probe to its backside where the material is consolidated to create a high-quality solid-state weld. The maximum temperature reached is of the order of $80 \%$ of the melting temperature. Despite the simplicity of the procedure, the mechanisms behind the process and the material flow around the probe tool are very complex. The material is extruded around the rotating tool and a vortex flow field near the probe due to the downward flow is induced by the probe thread. The process can be regarded as a solid phase keyhole welding technique since a 
hole to accommodate the probe is generated, then filled during the welding sequence. The material flow depends on welding process parameters, such as welding and rotation speed, pressure, etc., and on the characteristics of the tools, such as materials, design, etc.

The first applications of FSW have been in aluminium fabrications. Aluminium alloys that are difficult to weld using conventional welding techniques, are successfully welded using FSW. The weld quality is excellent, with none of the porosity that can arise in fusion welding, and the mechanical properties are at least as good as the best achievable by fusion welding. Being a solid-state welding process, the structure in the weld nugget is free of solidifying segregation, being suitable for welding of composite materials. The process is environmentally friendly, because no fumes or spatter are generated, and there is no arc glare or reflected laser beams with which to contend. Another major advantage is that, by avoiding the creation of a molten pool which shrinks significantly on re-solidification, the distortion after welding and the residual stresses are low. With regard to joint fit up, the process can accommodate a gap of up to $10 \%$ of the material thickness without impairing the quality of the resulting weld. As far as the rate of processing is concerned, for materials of $2 \mathrm{~mm}$ thickness, welding speeds of up to 2 $\mathrm{m} / \mathrm{min}$ can be achieved, and for $5 \mathrm{~mm}$ thickness up to $0.75 \mathrm{~m} / \mathrm{min}$. Recent tool developments are confidently expected to improve on these figures.

Friction stir welding has been used to weld all wrought aluminium alloys, across the AA-2xxx, AA-5xxx, AA-6xxx and AA-7xxx series of alloys, some of which are bordering on


being classed as virtualy unveldable by fusion wer
dissimilar aluminium alloys, whereas fuston wel
the different alloys interacting to form celeterio
solidification from the molter weld pool. F components by joining dissimilar materials
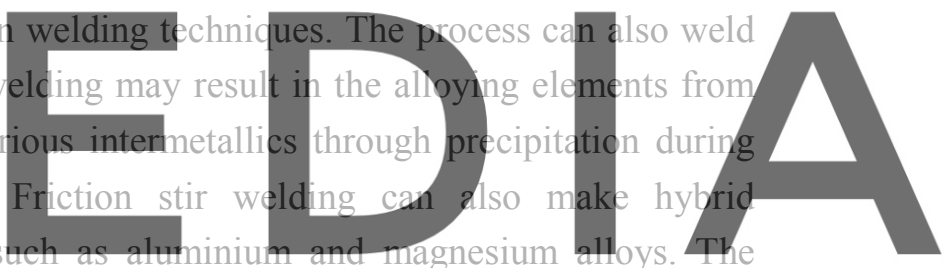

thicknesses of AA-6082-T6 that have so far been welded have ranged from $1.2 \mathrm{~mm}$ to $50 \mathrm{~mm}$ in

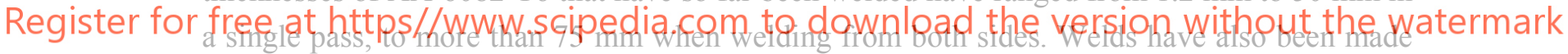

in pressure die cast aluminium material without any problems from pockets of entrapped high pressure gas, which would violently disrupt a molten weld pool encountering them.

The original application for friction stir welding was the welding of long lengths of material in the aerospace, shipbuilding and railway industries. Examples include large fuel tanks and other containers for space launch vehicles, cargo decks for high-speed ferries, and roofs for railway carriages. FSW is used already in routine, as well as in critical applications, for the joining of structural components made of aluminium and its alloys. Indeed, it has been convincingly demonstrated that the process results in strong and ductile joints, sometimes in systems which have proved difficult using conventional welding techniques. The process is most suitable for components which are flat and long (plates and sheets) but can be adapted for pipes, hollow sections and positional welding.

The computational modeling of FSW processes is a complex task and it has been a research topic of increasing interest in computational mechanics during the last decades.

Thermal models for the numerical simulation of FSW processes were used by McClure et al. (1998) [66], Colegrove et al. (2000) [46], and Khandkar and Khan $(2001,2003)[62,63]$. 
Bendzsak et al. $(2000)[15,16]$ used the Eulerian code Stir3D to model the flow around a FSW tool, including the tool thread and tilt angle in the tool geometry and obtaining complex flow patterns. The temperature effects on the viscosity were neglected.

Dong et al. (2001) [53] developed a simplified model for the numerical simulation of FSW processes, taking into account both the friction heating and plastic work in the modelling of the heat flow phenomena, predicting the development of a plastic strain around the weld zone in the initial stage of welding. However, they did not consider the longitudinal movement of the tool.

$\mathrm{Xu}$ et al. (2001) [79] and Xu and Deng (2003,2004) [80,81] developed a 3D finite element procedure to simulate the FSW process using the commercial Finite Element Method (FEM) software ABAQUS, focusing on the velocity field, the material flow characteristics and the equivalent plastic strain distribution. The authors used an Arbitrary Lagrangian-Eulerian (ALE) formulation with adaptive meshing and consider large elasto-plastic deformations and temperature-dependent material properties. However, the authors did not perform a fully coupled thermo-mechanical simulation, superimposing the temperature map obtained from the experiments as a prescribed temperature field to perform the mechanical analysis. The numerical results were compared to experimental data available, showing a reasonable good correlation between the equivalent plastic strain distributions and the distribution of the microstructure zones in the weld.


the discrepancies were explained by an inadequate representation of the constitutive behavior of

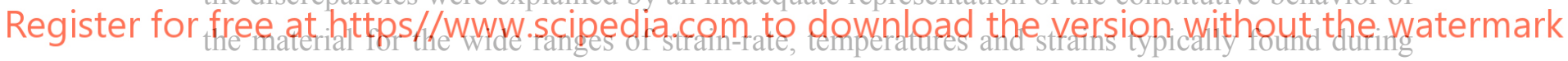
FSW.

Askari et al. (2003) [13] used the CTH hydrocode coupled to an advection-diffusion solver for the energy balance equation. The CTH code, developed by Sandia National Laboratories, uses the finite volume method to discretize the domain. The elastic response was taken into account in this case. The results proved encouraging with respect to gaining an understanding of the material flow around the tool. However, simplified friction conditions were used.

Chen and Kovacevic (2003) [28] developed a 3D FEM model to study the thermal history and thermo-mechanical phenomena in the butt-welding of aluminium alloy AA-6061-T6 using the commercial FEM code ANSYS. Their model incorporated the mechanical reaction between the tool and the weld material. Experiments were conducted and an X-ray diffraction technique was used to measure the residual stress in the welded plate. The welding tool (i.e. the shoulder and pin) in the FEM model was modeled as a heat source, with the nodes moved forward at each computational time step. This simple model severely limited the accuracy of the stress and force predictions.

Colegrove et al. $(2000,2004)$ [46,47] used the commercial Computational Fluid Dynamics (CFD) software FLUENT for a 2D and 3D numerical investigation on the influence 
of pin geometry during FSW, comparing different pin shapes in terms of material flow and welding forces on the basis of both a stick and a slip boundary condition at the tool/work-piece interface. In spite of the good obtained results, the accuracy of the analysis was limited by the assumption of isothermal conditions. Seidel and Reynolds (2003) [73] also used the CFD commercial software FLUENT to model the 2D steady-state flow around a cylindrical tool.

Schmidt and Hattel (2004) [72] presented the development of a 3D fully coupled thermo mechanical finite element model in ABAQUS/Explicit using the ALE formulation. The flexibility of the FSW machine was taken into account by connecting the rigid tool to a spring. The work-piece was modeled as a cylindrical volume with inlet and outlet boundary conditions. A rigid back-plate was used. The contact forces were modeled using a Coulomb friction law, and the surface was allowed to separate. Heat generated by friction and plastic deformation was considered. The simulation modeled the dwell and weld phases of the process.

An ALE formulation for the numerical simulation of FSW processes was also used by Zhao (2005) [82].

Nikiforakis (2005) [67] used a finite difference method to model the FSW process. Despite the fact that he was only presenting 2D results, the model proposed had the advantage of minimizing the calibration of model parameters, taking into account a maximum of physical effects. A transient and fully coupled thermo-fluid analysis was performed. The rotation of the tool was handled through the use of the overlapping grid method. A rigid-viscoplastic material

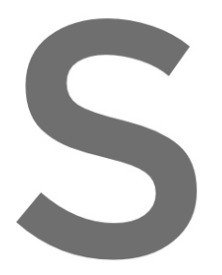
law was used and sticking contact at the tool w
was due to plastic deformation only.
Heurtier et al. (2006) [56] used a 3D
model to simulate FSW processes. The model heat input from the tool shoulder and $\mathrm{p}$
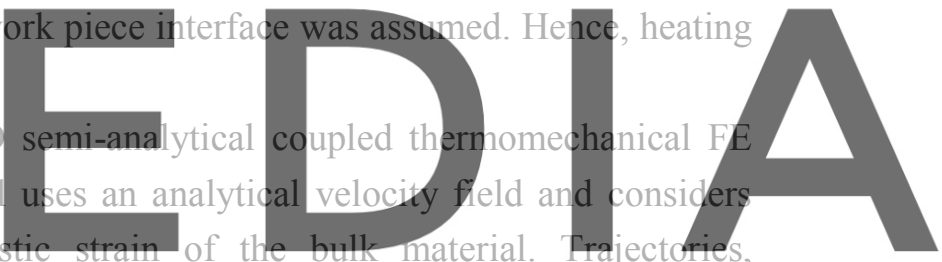

temperature, strain, strain rate fields and micro-hardness in various weld zones were computed

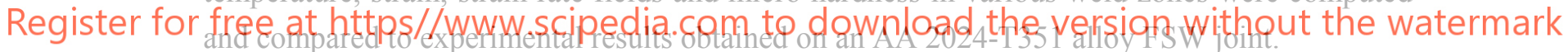

Buffa et al. (2006) [18] using the commercial finite element software DEFORM-3D,

proposed a 3D Lagrangian, implicit, coupled thermo-mechanical numerical model for the simulation of FSW processes, using a rigid-viscoplastic material description and a continuum assumption for the weld seam. The proposed model is able to predict the effect of process parameters on process variables, such as the temperature, strain and strain rate fields, as well as material flow and forces. A reasonable good agreement between the numerically predicted results, on forces and temperature distribution, and experimental data was obtained. The authors found that the temperature distribution about the weld line is nearly symmetric because the heat generation during FSW is dominated by rotating speed of the tool, which is much higher than the advancing speed. On the other hand, the material flow in the weld zone is nonsymmetrically distributed about the weld line because the material flow during FSW is mainly controlled by both advancing and rotating speeds.

De Vuyst et al. (2004-2006) [48,49,50,51] used the coupled thermo-mechanical finite element code MORFEO to simulate the flow around simplified tool geometries for FSW process. The rotation and advancing speed of the tool were modeled using prescribed velocity fields. An attempt to consider features associated to the geometrical details of the probe and shoulder, which had not been discretized in the finite element model in order to avoid very large 
meshes, was taken into account using additional special velocity boundary conditions. In spite of that, a mesh of roughly 250,000 nodes and almost 1.5 million of linear tetrahedral elements was used. A Norton-Hoff rigid-viscoplastic constitutive equation was considered, with averaged values of the consistency and strain rate sensitivity constitutive parameters determined from hot torsion tests performed over a range of temperatures and strain rates. The computed streamlines were compared with the flow visualization experimental results obtained using copper marker material sheets inserted transversally or longitudinally to the weld line. The simulation results correlated well when compared to markers inserted transversely to the welding direction. However, when compared to a marker inserted along the weld centerline only qualitative results could be obtained. The correlation may be improved by modeling the effective weld thickness of the experiment, using a more realistic material model, for example, by incorporating a yield stress or temperature dependent properties, refine velocity boundary conditions or further refining the mesh in specific zones, such as for instance, under the probe. The authors concluded that it is essential to take into account the effects of the probe thread and shoulder thread in order to get realistic flow fields.

Shercliff et al. (2005) [74] developed microstructural models for friction stir welding of 2000 series aluminium alloys.

López et al. (2008) [65] and Agelet de Saracibar et al. (2013) [9] developed numerical algorithms to optimize material model and FSW process parameters using neural networks.


They proposed a ndw model for the dissolution
alloys and they optimized the master curve and
they developed an algorithm to optrimize the ad
quality criteria the ninimization of the maximum
the pin.

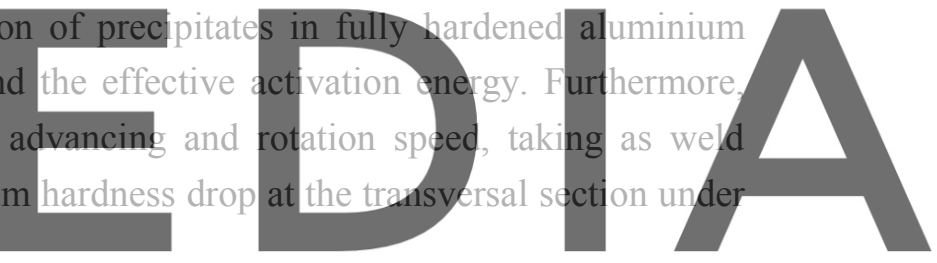

Santiago et al. (2010) [71] developed a simplified computational model taking into

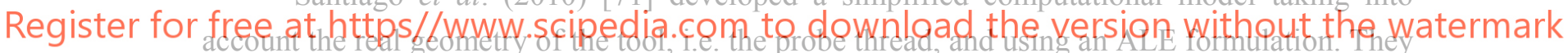
considered also a simplified friction model to take into account different slip/stick conditions at the pin shoulder/work-piece interface.

Chiumenti et al. (2012) [33], Dialami et al. (2012) [52], and Chiumenti et al. (2013) [34] developed an apropos kinematic framework for the numerical simulation of FSW processes. They considered a combination of ALE, Eulerian and Lagrangian descriptions at different zones of the computational domain and they proposed an efficient coupling strategy. Within this approach, a Lagrangian formulation was used for the pin, an ALE formulation was used at the stir zone of the work-piece, and an Eulerian formulation was used in the remaining part of the work-piece. The stir zone was defined as a circular domain close to the pin. The finite element mesh in the stir zone was rotating attached to the pin. The resulting apropos kinematic setting efficiently permitted to treat arbitrary pin geometries and facilitates the application of boundary conditions. The formulation was implemented in an enhanced version of the finite element code COMET [21] developed by the authors at the International Center for Numerical Methods in Engineering (CIMNE).

Bussetta et al. (2013) [19,20] compared a fluid approach using an apropos kinematic framework, developed by Chiumenti et al. (2012) [33], Dialami et al. (2012) [52], and 
Chiumenti et al. (2013) [34], and their solid approach using an ALE formalism, in the numerical simulation of FSW processes with a non-cylindrical pin.

This paper deals with the computational modeling and sub-grid scale stabilization of incompressibility and convection for the numerical simulation of the material flow around the probe tool in a FSW process.

Within the paradigmatic framework of the multiscale stabilization methods, suitable sub-grid scale finite element stabilization methods to stabilize the incompressibility constraint and the convection term arising in coupled thermomechanical formulations when a Eulerian description and mixed P1/P1/P1 linear velocity/linear pressure/linear temperature finite elements are used. Norton-Hoff and Sheppard-Wright thermo-rigid-viscoplastic constitutive material models are considered. Quasi-static sub-grid scales are considered and approximations of the sub-grid scale variables and stabilization parameters are given. In particular, Algebraic Sub-grid Scale (ASGS) and Orthogonal Sub-grid Scale (OSGS) methods are presented. Furthermore, it is shown that well known classical stabilized formulations, such as the Galerkin Least-Squares (GLS) for incompressible (or quasi-incompressible) problems or the Streamline Upwind/Petrov-Galerkin (SUPG) for convection dominant problems, can be recovered as particular eases of the multiscale stabilization framework considered.

Within the framework of fractional step methods, a product formula algorithm, arising from an isothermal split, is introduced to solve the coupled thermomechanical problem
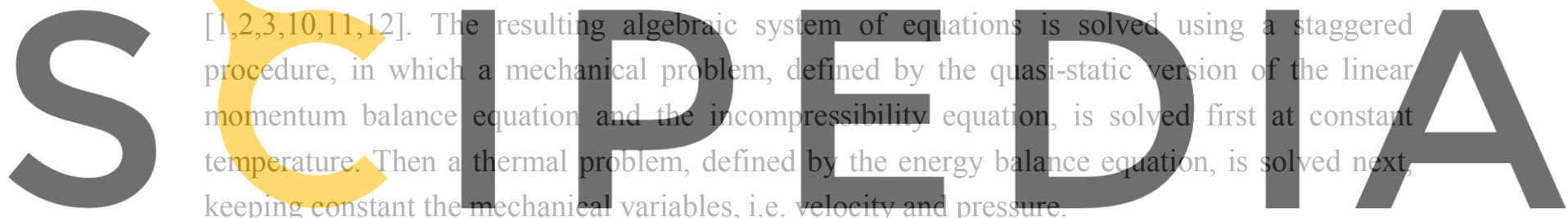

One of the goals of the paper is to compare the two sub-grid scale finite element

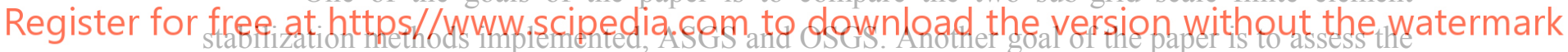
performance of the ASGS and OSGS methods in a 3D numerical simulation of a FSW process.

The outline of the paper is as follows. Section 2 deals with the sub-grid scale stabilized mixed formulation for the numerical simulation of the flow of the material around a tool probe in a FSW processes. Some key features of the FSW process are addressed and their implications in the computational model are first identified. The strong form of the governing equations and constitutive equations are provided, and the mixed variational form of the governing equations is given. Next, the multiscale stabilization method is introduced and an approximation of the sub-grid scale variables and the stabilization parameters is given. ASGS and OSGS finite element stabilization methods are considered and it is shown how classical GLS and SUPG methods can be recovered as a particular case of the ASGS method.

An isothermal operator split and a Product Formula Algorithm (PFA), defining mechanical and thermal problems, are introduced in Section 3. The ASGS and OSGS finite element stabilized formulation for the mechanical and thermal problems are presented.

Section 4 introduces a finite element partition of the mechanical and thermal problems and the resulting algebraic systems of equations are written in matrix form. Finally a convenient and efficient staggered solution algorithm for the OSGS method is presented. 
Two representative numerical examples are shown in Section 5. The first one is a numerical simulation of a coupled thermomechanical flow in a 2D rectangular domain. Steadystate and transient conditions are considered. An assessment of the ASGS and OSGS stabilization methods implemented is performed. The second example shows a 3D numerical simulation of a FSW process. Numerical results are compared with the experimental results provided by Zhu and Chao (2004) [83]. Calculations are performed using an enhanced version of the finite element program COMET [21] developed by the authors at the International Center for Numerical Methods in Engineering (CIMNE) in Barcelona. Pre- and post-processing is done with GiD [54], also developed at CIMNE. Finally some concluding remarks are drawn.

\section{Sub-grid scale stabilized mixed formulation}

\subsection{Computational modelling of FSW processes}

The flow of the material around a FSW tool is characterized by a Reynolds number which is much smaller than 1 , typically of the order $10^{-4}$, due to the small length scale, the low velocities and the very high viscosity of the material. For these values of the Reynolds number, the inertial forces of the linear momentum balance equation can be neglected and a quasi-static analysis can be performed.

The deformation of the material taking place around a FSW tool is extremely high. The computational modeling of the material flow around a FSW tool using a Lagrangian formulation requires continuous remeshing to avoid extremely distorted mesh elements. Therefore, the use of alternative formulations, such as ALE or Eulerian formulations, is a better choice. In this work we will use an Eulerian formulation.

Transient thermal conditions will be considered. On the other hand, the Peclet number for a FSW process typically ranges from $10^{1}$ to $10^{3}$. For this range of values of the Peclet number, the convective term of the spatial energy balance equation cannot be neglected.

Coupled thermo-mechanical rigid-visco-plastic constitutive material models, such as the Norton-Hoff or the Sheppard-Wright, will be considered [6,7,8,19,20,33,34,52].

Heat transfer by conduction, convection and radiation are considered. Heat transfer by conduction is considered at the contact interface between the work-pieces and the tool, and the work-pieces and the back-plate. Heat transfer by convection, governed by the Newton law, and radiation, governed by the Stefan-Boltzmann law, are considered at the surfaces of the workpieces and the tool which are in contact with the environment [33,34].

The resulting coupled thermo-mechanical problem will be solved using a product formula algorithm, arising from an isothermal split, leading to a staggered solution algorithm. A mechanical problem, involving mechanical variables as unknowns, is defined at constant temperature and a thermal problem, involving the temperature as unknown, is defined at constant configuration. A pressure stabilized mixed linear velocity/linear pressure finite element interpolation formulation will be used to solve the mechanical problem and a convection stabilized linear temperature interpolation formulation will be used to solve the thermal problem $[33,34]$.

\subsection{Local formulation}


Let $\Omega \subset \mathbb{R}^{n_{\text {dim }}}$, with $n_{\text {dim }}=2,3$, be the computational domain of interest in which the deformation takes place during the time interval $[0, T]$, and let $\partial \Omega$ be its boundary. Let us consider the following partitions of the boundary $\partial \Omega=\partial_{\nu} \Omega \cup \partial_{\sigma} \Omega, \partial \Omega=\partial_{\vartheta} \Omega \cup \partial_{q} \Omega$, such that $\varnothing=\partial_{v} \Omega \cap \partial_{\sigma} \Omega, \varnothing=\partial_{\vartheta} \Omega \cap \partial_{q} \Omega$. Using a mixed velocity/pressure/temperature spatial formulation, considering quasi-static conditions and incompressibility, the Initial and Boundary Value Problem (IBVP) to be considered is defined by the local spatial form of the linear momentum balance equation, mass continuity equation and energy balance equation, together with appropriate Dirichlet and Neumann boundary conditions, and initial conditions. The IBVP consists in finding a velocity vector field $\mathbf{v}$, a pressure scalar field $p$, and a temperature scalar field $\vartheta$ such that

$$
\begin{aligned}
-\nabla p-\nabla \cdot \mathbf{s}(\mathbf{v}, \vartheta) & =\mathbf{b} & & \text { in } \Omega, t \in[0, T] \\
\nabla \cdot \mathbf{v} & =\mathbf{0} & & \text { in } \Omega, t \in[0, T] \\
c \partial_{t} \vartheta+c \mathbf{v} \cdot \nabla \vartheta+\nabla \cdot \mathbf{q}(\vartheta) & =r+\mathcal{D} & & \text { in } \Omega, t \in[0, T] \\
\mathbf{v} & =\overline{\mathbf{v}} & & \text { on } \partial_{v} \Omega, t \in[0, T] \\
(p \mathbf{1}+\mathbf{s}(\mathbf{v}, \vartheta)) \mathbf{n} & =\overline{\mathbf{t}} & & \text { on } \partial_{\sigma} \Omega, t \in[0, T] \\
\vartheta & =\bar{\vartheta} & & \text { on } \partial_{\vartheta} \Omega, t \in[0, T] \\
\mathbf{q}(\vartheta) \cdot \mathbf{n} & =\bar{q} & & \text { on } \partial_{q} \Omega, t \in[0, T] \\
\vartheta & =\vartheta_{0} & & \text { in } \Omega, t=0
\end{aligned}
$$

In these equations, $\mathbf{s}(\mathbf{v}, \vartheta)$ is the deviatoric part of the Cauchy stress tensor, $\mathbf{q}(\vartheta)$ is the spatial heat flux per unit of surface, $\mathbf{b}$ is the body force per unit of spatial volume, $r$ is the internal heat source rate per unit of spatial volume, $\mathcal{D}=\mathcal{D}(\mathbf{v}, \vartheta)$ is the dissipation rate per unit of spatial volume, $\overline{\mathbf{v}}$ is the prescribed velocity vector field, $\overline{\mathbf{t}}$ is the prescribed traction vector field, $\bar{\vartheta}$ is the prescribed temperature, $\bar{q}$ is the prescribed outward normal heat flux per unit of surface, $\vartheta_{0}$ is the initial temperature, $c=c(\vartheta)$ is the heat capacity, $\nabla$ is the spatial nabla vector differential operator, and $\mathbf{1}$ is the unit second-order tensor.

Heat transfer by thermal contact, convection and radiation is considered according to the following expressions

$$
\begin{aligned}
\bar{q}_{c} & =h_{c} g_{\vartheta} \\
\bar{q}_{c o n v} & =h_{c o n v}\left(\vartheta-\vartheta_{e n v}\right) \\
\bar{q}_{\text {rad }} & =\sigma \varepsilon\left(\vartheta^{4}-\vartheta_{e n v}^{4}\right)
\end{aligned}
$$

where $\bar{q}_{c}$ is the heat transfer by thermal contact, $\bar{q}_{c o n v}$ is the heat transfer by convection, $\bar{q}_{\text {rad }}$ is the heat transfer by radiation, $h_{c}$ is the contact heat transfer coefficient, $g_{\vartheta}$ is the thermal contact gap, $h_{\text {conv }}$ is the convection heat transfer coefficient, $\vartheta_{e n v}$ is the environmental temperature, $\sigma=5.6704 \mathrm{E}-08 \mathrm{~W} \mathrm{~m}^{-2} \mathrm{~K}^{-4}$ is the Stefan-Boltzmann constant, and $\varepsilon$ is the relative emissivity.

Material mechanical response is modeled using an incompressible thermo-rigid-plastic material model. Taking into account incompressibility, the constitutive equation for the 
deviatoric part of the Cauchy stress tensor and the dissipation rate per unit of volume can be written as

$$
\begin{aligned}
\mathbf{s}(\mathbf{v}, \vartheta) & =2 \mu(\mathbf{v}, \vartheta) \operatorname{dev}\left(\nabla^{s} \mathbf{v}\right)=2 \mu(\mathbf{v}, \vartheta) \nabla^{s} \mathbf{v} \\
\mathcal{D}(\mathbf{v}, \vartheta) & =\mathbf{s}(\mathbf{v}, \vartheta): \operatorname{dev}\left(\nabla^{s} \mathbf{v}\right)=2 \mu(\mathbf{v}, \vartheta) \operatorname{dev}\left(\nabla^{s} \mathbf{v}\right): \operatorname{dev}\left(\nabla^{s} \mathbf{v}\right)=2 \mu(\mathbf{v}, \vartheta) \nabla^{s} \mathbf{v}: \nabla^{s} \mathbf{v}
\end{aligned}
$$

where $\mu=\mu(\mathbf{v}, \vartheta)$ is the equivalent viscosity of the material model, $\nabla^{s}(\cdot)$ is the spatial symmetric gradient operator, and $\operatorname{dev}(\cdot)$ is the deviatoric operator.

Here two target thermo-rigid-plastic material models, Norton-Hoff and SheppardWright, will be considered and their constitutive equations are given in the next sub-section.

Material thermal response is modeled using Fourier law given by

$$
\mathbf{q}(\vartheta)=-\kappa(\vartheta) \nabla \vartheta
$$

where $\kappa=\kappa(\vartheta)$ is the thermal conductivity.

Substituting the mechanical and thermal constitutive equations into the governing equations, the IBVP can be written as

$$
\begin{aligned}
-\nabla p-\nabla \cdot\left(2 \mu \nabla^{s} \mathbf{v}\right) & =\mathbf{b} & & \text { in } \Omega, t \in[0, T] \\
\nabla \cdot \mathbf{v} & =\mathbf{0} & & \text { in } \Omega, t \in[0, T] \\
c \partial_{t} \vartheta+c \mathbf{v} \cdot \nabla \vartheta-\nabla \cdot(\kappa \nabla \vartheta) & =r+\mathcal{D} & & \text { in } \Omega, t \in[0, T] \\
\mathbf{v} & =\overline{\mathbf{v}} & & \text { on } \partial_{v} \Omega, t \in[0, T] \\
\left(p \mathbf{1}+2 \mu \nabla^{s} \mathbf{v}\right) \mathbf{n} & =\overline{\mathbf{t}} & & \text { on } \partial_{\sigma} \Omega, t \in[0, T] \\
\vartheta & =\bar{\vartheta} & & \text { on } \partial_{\vartheta} \Omega, t \in[0, T] \\
-\kappa \nabla \vartheta \cdot \mathbf{n} & =\bar{q} & & \text { on } \partial_{q} \Omega, t \in[0, T] \\
\vartheta & =\vartheta_{0} & & \text { in } \Omega, t=0
\end{aligned}
$$

\subsection{Constitutive equations}

Two mechanical constitutive incompressible thermo-rigid-plastic material models, suitable for the numerical simulation of Friction Stir Welding (FSW) processes, are considered: NortonHoff and Sheppard-Wright [6,7,8,19,20,33,34,52].

(i) Norton-Hoff material model: The constitutive equation for the rigid-plastic NortonHoff material model is given by

$$
\mathbf{s}(\mathbf{v}, \vartheta)=2 \mu(\mathbf{v}, \vartheta) \operatorname{dev}\left(\nabla^{s} \mathbf{v}\right)=2 \mu(\mathbf{v}, \vartheta) \nabla^{s} \mathbf{v}:=K(\vartheta)(\sqrt{3} \dot{\bar{\varepsilon}}(\mathbf{v}))^{m(\vartheta)-1} \nabla^{s} \mathbf{v}
$$

where $K(\vartheta)$ is a temperature dependent consistency parameter, $0 \leq m(\vartheta) \leq 1$ is a temperature dependent rate sensitivity parameter and $\dot{\bar{\varepsilon}}(\mathbf{v})$ is the equivalent strain rate defined as 


$$
\dot{\bar{\varepsilon}}(\mathbf{v})=\left(\frac{2}{3} \operatorname{dev}\left(\nabla^{s} \mathbf{v}\right): \operatorname{dev}\left(\nabla^{s} \mathbf{v}\right)\right)^{1 / 2}=\left(\frac{2}{3} \nabla^{s} \mathbf{v}: \nabla^{s} \mathbf{v}\right)^{1 / 2}
$$

Then, the equivalent viscosity for the Norton-Hoff material model takes the form

$$
\mu(\mathbf{v}, \vartheta):=\frac{1}{2} K(\vartheta)(\sqrt{3} \dot{\bar{\varepsilon}}(\mathbf{v}))^{m(\vartheta)-1}
$$

(ii) Sheppard-Wright material model: The constitutive equation for the rigid-plastic Sheppard-Wright material model is given by

$$
\mathbf{s}(\mathbf{v}, \vartheta)=2 \mu(\mathbf{v}, \vartheta) \operatorname{dev}\left(\nabla^{s} \mathbf{v}\right)=2 \mu(\mathbf{v}, \vartheta) \nabla^{s} \mathbf{v}:=\frac{2 \sigma_{e}(\dot{\bar{\varepsilon}}(\mathbf{v}), \vartheta)}{3 \dot{\bar{\varepsilon}}(\mathbf{v})} \nabla^{s} \mathbf{v}
$$

where $\sigma_{e}(\dot{\bar{\varepsilon}}(\mathbf{v}), \vartheta)$ is the strain rate and temperature dependent yield stress defined as

$$
\sigma_{e}(\dot{\bar{\varepsilon}}(\mathbf{v}), \vartheta)=\frac{1}{\alpha} \log \left[\left(\frac{Z(\dot{\bar{\varepsilon}}(\mathbf{v}), \vartheta)}{A}\right)^{1 / n}+\sqrt{1+\left(\frac{Z(\dot{\bar{\varepsilon}}(\mathbf{v}), \vartheta)}{A}\right)^{2 / n}}\right]
$$

where $\alpha, A$ and $n$ are material parameters, and $Z(\dot{\bar{\varepsilon}}(\mathbf{v}), \vartheta)$ is the Zener-Hollomon parameter, representing the temperature compensated equivalent strain rate, given by

$$
Z(\dot{\bar{\varepsilon}}(\mathbf{v}), \vartheta)=\dot{\bar{\varepsilon}}(\mathbf{v}) \exp \left(\frac{Q}{R \vartheta}\right)
$$

where $Q$ is the activation energy and $R=8.314 \mathrm{~J} \mathrm{~mol}^{-1} \mathrm{~K}^{-1}$ is the universal constant for an ideal gas.

Then, the equivalent viscosity for the Sheppard-Wright material model takes the form

$$
\mu(\mathbf{v}, \vartheta):=\frac{\sigma_{e}(\dot{\bar{\varepsilon}}(\mathbf{v}), \vartheta)}{3 \dot{\bar{\varepsilon}}(\mathbf{v})}
$$

\subsection{Variational formulation}

To define the variational setting let us introduce some standard notation. Let $L^{p}(\Omega)$ be the space of functions whose $p$ power $(1 \leq p<\infty)$ are integrable in a domain $\Omega$, being $L^{\infty}(\Omega)$ the space of bounded functions in $\Omega$. Let $H^{m}(\Omega)$ be the space of functions whose derivatives of order up to $m \geq 0$ (integer) belong to $L^{2}(\Omega)$. The space $H_{0}^{1}(\Omega)$ consists of functions in $H^{1}(\Omega)$ vanishing on the boundary. A bold character is used to denote the vector counterpart of all these spaces. The $L^{2}(\Omega)$ inner product is denoted as $(\cdot, \cdot)$ and the $L^{2}(\partial \Omega)$ inner product is denoted as $(\cdot, \cdot)_{\partial \Omega}$.

Let us introduce the infinite dimensional functional spaces $\mathcal{W}:=\mathcal{V} \times \mathcal{P} \times \mathcal{T}$ and $\mathcal{W}_{0}:=\mathcal{V}_{0} \times \mathcal{Q} \times \mathcal{T}_{0}$ where 


$$
\begin{aligned}
& \mathcal{V}=\left\{\mathbf{v} \in \boldsymbol{H}^{2}(\Omega) \mid \mathbf{v}=\overline{\mathbf{v}} \text { on } \partial_{u} \Omega\right\} \\
& \mathcal{P}=\left\{p \in H^{1}(\Omega)\right\} \\
& \mathcal{T}=\left\{\vartheta \in H^{2}(\Omega) \mid \vartheta=\bar{\vartheta} \text { on } \partial_{\vartheta} \Omega\right\} \\
& \mathcal{V}=\left\{\delta \mathbf{v} \in \boldsymbol{L}^{2}(\Omega) \mid \delta \mathbf{v}=\mathbf{0} \text { on } \partial_{u} \Omega\right\} \\
& \mathcal{Q}=\left\{\delta p \in L^{2}(\Omega)\right\} \\
& \mathcal{T}_{0}=\left\{\delta \vartheta \in L^{2}(\Omega) \mid \delta \vartheta=0 \text { on } \partial_{\vartheta} \Omega\right\}
\end{aligned}
$$

The variational form of the IBVP consists in finding a velocity vector field $\mathbf{v} \in \mathcal{V}$, pressure scalar field $p \in \mathcal{P}$ and temperature scalar field $\vartheta \in \mathcal{T}$ such that

$$
\begin{aligned}
-(\delta \mathbf{v}, \nabla p)-\left(\delta \mathbf{v}, \nabla \cdot\left(2 \mu \nabla^{s} \mathbf{v}\right)\right) & =(\delta \mathbf{v}, \mathbf{b}) \quad \forall \delta \mathbf{v} \\
(\delta p, \nabla \cdot \mathbf{v}) & =0 \quad \forall \delta p \\
\left(\delta \vartheta, c \partial_{t} \vartheta\right)+(\delta \vartheta, c \mathbf{v} \cdot \nabla \vartheta)-(\delta \vartheta, \nabla \cdot(\kappa \nabla \vartheta)) & =(\delta \vartheta, r)+(\delta \vartheta, \mathcal{D}) \quad \forall \delta \vartheta
\end{aligned}
$$

where $\delta \mathbf{v} \in \mathcal{V}_{0}, \delta p \in \mathcal{Q}, \delta \vartheta \in \mathcal{T}_{0}$.

Integrating by parts some of the terms above, the variational form of the IBVP consists in finding a velocity vector field $\mathbf{v} \in \mathcal{V}$, pressure scalar field $p \in \mathcal{P}$ and temperature scalar field $\vartheta \in \mathcal{T}$ such that

$$
\begin{aligned}
(\nabla \cdot \delta \mathbf{v}, p)+\left(\nabla^{s} \delta \mathbf{v}, 2 \mu \nabla^{s} \mathbf{v}\right) & =F_{v}(\delta \mathbf{v}) \quad \forall \delta \mathbf{v} \\
(\delta p, \nabla \cdot \mathbf{v}) & =0 \quad \forall \delta p \\
\left(\delta \vartheta, c \partial_{t} \vartheta\right)+(\delta \vartheta, c \mathbf{v} \cdot \nabla \vartheta)+(\nabla \delta \vartheta, \kappa \nabla \vartheta) & =F_{\vartheta}(\delta \vartheta) \quad \forall \delta \vartheta
\end{aligned}
$$

where

$$
\begin{aligned}
& F_{v}(\delta \mathbf{v}):=(\delta \mathbf{v}, \mathbf{b})+(\delta \mathbf{v}, \overline{\mathbf{t}})_{\partial_{\sigma} \Omega} \\
& F_{\vartheta}(\delta \vartheta):=(\delta \vartheta, r)+(\delta \vartheta, \mathcal{D})-(\delta \vartheta, \bar{q})_{\partial_{q} \Omega}
\end{aligned}
$$

and $\delta \mathbf{v} \in \mathcal{V}_{0}, \delta p \in \mathcal{P}, \delta \vartheta \in \mathcal{T}_{0}$ where, now the spaces of functions $\mathcal{W}:=\mathcal{V} \times \mathcal{P} \times \mathcal{T}$ and $\mathcal{W}_{0}:=\mathcal{V}_{0} \times \mathcal{P} \times \mathcal{T}_{0}$ are defined as

$$
\begin{aligned}
& \mathcal{V}=\left\{\mathbf{v} \in \boldsymbol{H}^{1}(\Omega) \mid \mathbf{v}=\overline{\mathbf{v}} \text { on } \partial_{v} \Omega\right\} \\
& \mathcal{P}=\left\{p \in L^{2}(\Omega)\right\} \\
& \mathcal{T}=\left\{\vartheta \in H^{1}(\Omega) \mid \vartheta=\bar{\vartheta} \text { on } \partial_{\vartheta} \Omega\right\} \\
& \mathcal{V}_{0}=\left\{\delta \mathbf{v} \in \boldsymbol{H}_{0}^{1}(\Omega) \mid \delta \mathbf{v} \in \boldsymbol{H}^{1}(\Omega) \text { and } \delta \mathbf{v}=\mathbf{0} \text { on } \partial_{v} \Omega\right\} \\
& \mathcal{T}_{0}=\left\{\delta \vartheta \in H_{0}^{1}(\Omega) \mid \delta \vartheta \in H^{1}(\Omega) \text { and } \delta \vartheta=0 \text { on } \partial_{\vartheta} \Omega\right\}
\end{aligned}
$$




\subsection{Galerkin finite element projection}

The standard Galerkin projection of the variational form of the IBVP is now straightforward. Let us consider a finite element partition $\left\{\Omega_{e}\right\}, e=1, \ldots, n_{e}$ of the computational domain $\Omega$, where $n_{e}$ is the number of elements in the partition. We can now construct conforming finite element spaces for the velocity, pressure and temperature in the usual manner. We will assume that they are all built from continuous piecewise polynomials of the same degree $k$.

Let us introduce the finite element spaces $\mathcal{W}_{h}:=\mathcal{V}_{h} \times \mathcal{P}_{h} \times \mathcal{T}_{h} \subset \mathcal{W}$ and $\mathcal{W}_{0, h}:=\mathcal{V}_{0, h} \times \mathcal{P}_{h} \times \mathcal{T}_{0, h} \subset \mathcal{W}_{0}$ where $\mathcal{V}_{h} \subset \mathcal{V}, \mathcal{P}_{h} \subset \mathcal{P}, \mathcal{T}_{h} \subset \mathcal{T}, \mathcal{V}_{0, h} \subset \mathcal{V}_{0}$ and $\mathcal{T}_{0, h} \subset \mathcal{T}_{0}$ are defined as

$$
\begin{aligned}
\mathcal{V}_{h} & =\left\{\mathbf{v}_{h} \in \boldsymbol{H}^{1}(\Omega) \mid \mathbf{v}_{h}=\overline{\mathbf{v}}_{h} \text { on } \partial_{v} \Omega\right\} \\
\mathcal{P}_{h} & =\left\{p_{h} \in L^{2}(\Omega)\right\} \\
\mathcal{T}_{h} & =\left\{\vartheta_{h} \in H^{1}(\Omega) \mid \vartheta_{h}=\bar{\vartheta}_{h} \text { on } \partial_{\vartheta} \Omega\right\} \\
\mathcal{V}_{0, h} & =\left\{\delta \mathbf{v}_{h} \in \boldsymbol{H}^{1}(\Omega) \mid \delta \mathbf{v}_{h}=\mathbf{0} \text { on } \partial_{v} \Omega\right\} \\
\mathcal{T}_{0, h} & =\left\{\delta \vartheta_{h} \in H^{1}(\Omega) \mid \delta \vartheta_{h}=0 \text { on } \partial_{\vartheta} \Omega\right\}
\end{aligned}
$$

The spatial discrete variational form of the IBVP consists in finding a velocity vector field $\mathbf{v}_{h} \in \mathcal{V}_{h}$, pressure scalar field $p_{h} \in \mathcal{P}_{h}$ and temperature scalar field $\vartheta_{h} \in \mathcal{T}_{h}$ such that

$$
\begin{aligned}
\left(\nabla \cdot \delta \mathbf{v}_{h}, p_{h}\right)+\left(\nabla^{s} \delta \mathbf{v}_{h}, 2 \mu_{h} \nabla^{s} \mathbf{v}_{h}\right) & =F_{v}\left(\delta \mathbf{v}_{h}\right) \quad \forall \delta \mathbf{v}_{h} \\
\left(\delta p_{h}, \nabla \cdot \mathbf{v}_{h}\right) & =0 \quad \forall \delta p_{h} \\
\left(\delta \vartheta_{h}, c_{h} \partial_{t} \vartheta_{h}\right)+\left(\delta \vartheta_{h}, c_{h} \mathbf{v}_{h} \cdot \nabla \vartheta_{h}\right)+\left(\nabla \delta \vartheta_{h}, \kappa_{h} \nabla \vartheta_{h}\right) & =F_{\vartheta}\left(\delta \vartheta_{h}\right) \quad \forall \delta \vartheta_{h}
\end{aligned}
$$

where $\delta \mathbf{v}_{h} \in \mathcal{V}_{0, h}, \delta p_{h} \in \mathcal{P}_{h}, \delta \vartheta_{h} \in \mathcal{T}_{0, h}$.

It is well know that the spatial discrete variational problem defined above may suffer from two types of numerical instabilities: the compatibility required for the velocity and pressure finite element spaces posed by the inf-sup condition and the dominance of the (nonlinear) convective term over the viscous one when the equivalent viscosity is small [17].

In this work we adopt a stabilized finite element formulation based on the sub-grid scales method first introduced by Hughes (1995) [58] and as a Variational Multiscale (VMS) stabilization method by Hughes et al. (1998) [59]. The sub-grid scale finite element stabilization method allows the use of equal velocity/pressure order interpolation, thus avoiding or bypassing the need to satisfy the inf-sup or Babuska-Brezzi condition [17], and avoids the oscillations which arise in convective dominant problems. The basic idea is to split the exact continuous unknowns into two components, corresponding to two different scales, the resolvable coarse scale and the unresolved fine scale. The first one is captured by the Galerkin finite element projection and is denoted as the finite element scale. The second one cannot be captured by the finite element solution and is denoted as the sub-grid scale. The goal is to find an approximate solution for the sub-grid scales and to include them into the discrete finite element solution.

Within the sub-grid scales framework, different stabilization formulations can be considered. Here we will restrict our attention to two approaches denoted as Algebraic Sub-grid 
Scale (ASGS) and Orthogonal Sub-grid Scale (OSGS). In the ASGS method, the velocity, pressure and temperature sub-grid scales are taken proportional to the corresponding residuals of the velocity, pressure and temperature equations, given in terms of the linear momentum balance, incompressibility and energy balance equations, respectively. In the OSGS method, only the component of these residuals which is $L^{2}$ orthogonal to the corresponding finite element space is considered. This idea was first introduced by Codina (2000) in [39] as an extension of a stabilization method originally introduced for the Stokes problem in [36] and fully analyzed for the stationary Navier-Stokes equations in [40].

The main features of the sub-grid scale finite element stabilization formulation proposed in this work are the following: (i) quasi-static sub-grid scales, instead of dynamic sub-grid scales, are considered, neglecting the time derivative of the sub-grid scale temperature; (ii) only sub-grid scale velocity and temperature are introduced, assuming that the sub-grid scale pressure is zero; (iii) the stabilized velocity, instead of its finite element component, is used in the convective term of the sub-grid scale temperature; (iv) tracking of the sub-grid scales is not done in the temperature dependent material properties and the dissipation rate, and they are approximated by their corresponding finite element components; (v) ASGS and OSGS sub-grid scale finite element stabilization methods are considered.

\subsection{Sub-grid scales stabilization}

Within the paradigmatic sub-grid scale stabilization framework introduced by Hughes et al. (1998) [57], it is considered that the continuous unknown fields can be split in two components, corresponding to different scales or levels of resolution: a coarse one, which is captured by the finite element partition, and a fine one, which cannot be captured by the finite element partition.

In order to get a stable finite element solution for the discrete finite element formulation, it is necessary to include, somehow, the effect of both scales in the approximation. The coarse or finite element scale can be appropriately solved by a standard Galerkin finite element approximation, while the fine or sub-grid scale can be included, at least locally, to enhance the stability of the Galerkin finite element approximation.

Let us consider the following splits of the infinite dimensional spaces as $\mathcal{W}=\mathcal{W}_{h} \oplus \tilde{\mathcal{W}}$ and $\mathcal{W}_{0}=\mathcal{W}_{0, h} \oplus \tilde{\mathcal{W}}_{0}$, where $\tilde{\mathcal{W}}=\tilde{\mathcal{V}} \times \tilde{\mathcal{P}} \times \tilde{\mathcal{T}}$ is any suitable space to complete $\mathcal{W}_{h}$ in $\mathcal{W}$ and $\tilde{\mathcal{V}}_{0}=\tilde{\mathcal{V}_{0}} \times \tilde{\mathcal{P}_{0}} \times \tilde{\mathcal{T}_{0}}$ is any suitable space to complete $\mathcal{W}_{0, h}$ in $\mathcal{W}_{0}$, where $\tilde{\mathcal{V}}, \tilde{\mathcal{P}}, \tilde{\mathcal{T}}$ are sub-grid scale spaces for the velocity, pressure and temperature, respectively, and $\tilde{\mathcal{V}_{0}}, \tilde{\mathcal{P}_{0}}, \tilde{\mathcal{T}_{0}}$ are the sub-grid scale spaces for the variations of the velocity, pressure and temperature, respectively. Obviously the sub-grid scale spaces are infinitedimensional spaces, but once the final method is formulated they will be approximated by finite-dimensional spaces, although we will keep the same symbols in order to simplify the notation.

Let us consider the following sub-grid scale split of the exact continuous velocity, pressure and temperature fields

$$
\mathbf{v}=\mathbf{v}_{h}+\tilde{\mathbf{v}}, \quad p=p_{h}+\tilde{p}, \quad \vartheta=\vartheta_{h}+\tilde{\vartheta}
$$

where the components with subscripts $h$ belong to the corresponding finite element spaces, and the components with the tilde belong to the corresponding sub-grid spaces. Then 
$\mathbf{v}_{h} \in \mathcal{V}_{h}, p_{h} \in \mathcal{P}_{h}, \vartheta_{h} \in \mathcal{T}_{h}$ are the components of the velocity, pressure and temperature on the (coarser) finite element scale, and $\tilde{\mathbf{v}} \in \tilde{\mathcal{V}}, \tilde{p} \in \tilde{\mathcal{P}}, \tilde{\vartheta} \in \tilde{\mathcal{T}}$ are the components of the velocity, pressure and temperature on the (finer) sub-grid scale. These additional components are what we will call sub-grid scale or subscale velocity, pressure and temperature. We will assume that the sub-grid scales vanish at the inter-element boundaries $\partial \Omega_{e}$ and thus $\tilde{\mathcal{W}} \approx \tilde{W}_{0}$. This happens, for instance, if they are approximated using bubble functions [58], or if one assumes that their Fourier modes correspond to high wave numbers [39].

Substituting the sub-grid scale split of the velocity and temperature into the equivalent viscosity, thermal conductivity and heat capacity, the following expressions are obtained and the following approximations are introduced

$$
\begin{aligned}
& \mu=\mu\left(\mathbf{v}_{h}+\tilde{\mathbf{v}}, \vartheta_{h}+\tilde{\vartheta}\right) \approx \mu\left(\mathbf{v}_{h}, \vartheta_{h}\right):=\mu_{h} \\
& \kappa=\kappa\left(\vartheta_{h}+\tilde{\vartheta}\right) \approx \kappa\left(\vartheta_{h}\right):=\kappa_{h} \\
& c=c\left(\vartheta_{h}+\tilde{\vartheta}\right) \approx c\left(\vartheta_{h}\right):=c_{h}
\end{aligned}
$$

Substituting the sub-grid scale split of the velocity and temperature into the mechanical and thermal constitutive equations, and taking into account the above approximations, yields

$$
\begin{aligned}
\mathbf{s}(\mathbf{v}, \vartheta) & =2 \mu(\mathbf{v}, \vartheta) \operatorname{dev} \nabla^{s} \mathbf{v}=2 \mu(\mathbf{v}, \vartheta) \nabla^{s} \mathbf{v} \\
& \approx 2 \mu\left(\mathbf{v}_{h}, \vartheta_{h}\right) \nabla^{s} \mathbf{v}_{h}+2 \mu\left(\mathbf{v}_{h}, \vartheta_{h}\right) \nabla^{s} \tilde{\mathbf{v}}:=2 \mu_{h} \nabla^{s} \mathbf{v}_{h}+2 \mu_{h} \nabla^{s} \tilde{\mathbf{v}} \\
\mathbf{q}(\vartheta) & =-\kappa(\vartheta) \nabla \vartheta \\
& \approx-\kappa\left(\vartheta_{h}\right) \nabla \vartheta_{h}-\kappa\left(\vartheta_{h}\right) \nabla \tilde{\vartheta}:=-\kappa_{h} \nabla \vartheta_{h}-\kappa_{h} \nabla \tilde{\vartheta}
\end{aligned}
$$

Similarly, the following approximation is introduced for the dissipation rate per unit of volume

$$
\begin{aligned}
\mathcal{D}(\mathbf{v}, \vartheta) & =\mathbf{s}(\mathbf{v}, \vartheta): \operatorname{dev}\left(\nabla^{s} \mathbf{v}\right)=2 \mu(\mathbf{v}, \vartheta) \operatorname{dev}\left(\nabla^{s} \mathbf{v}\right): \operatorname{dev}\left(\nabla^{s} \mathbf{v}\right) \\
& =2 \mu(\mathbf{v}, \vartheta) \nabla^{s} \mathbf{v}: \nabla^{s} \mathbf{v} \\
& \approx 2 \mu\left(\mathbf{v}_{h}, \vartheta_{h}\right)\left(\nabla^{s} \mathbf{v}_{h}+\nabla^{s} \tilde{\mathbf{v}}\right):\left(\nabla^{s} \mathbf{v}_{h}+\nabla^{s} \tilde{\mathbf{v}}\right) \\
& \approx 2 \mu\left(\mathbf{v}_{h}, \vartheta_{h}\right) \nabla^{s} \mathbf{v}_{h}: \nabla^{s} \mathbf{v}_{h}:=2 \mu_{h} \nabla^{s} \mathbf{v}_{h}: \nabla^{s} \mathbf{v}_{h}:=\mathcal{D}\left(\mathbf{v}_{h}, \vartheta_{h}\right):=\mathcal{D}_{h}
\end{aligned}
$$

Neglecting the sub-grid scale pressure, $\tilde{p}=0$, considering quasi-static sub-grid scales, neglecting the dynamic sub-grid scale temperature, setting $\partial_{t} \tilde{\vartheta}=0$, and substituting the subgrid scale split of the velocity and temperature into the variational formulation of the IBVP, two systems of variational equations are obtained. The first one represents the projection of the governing equations onto the corresponding discrete finite element spaces, and is given by 


$$
\begin{aligned}
& \left(\nabla \cdot \delta \mathbf{v}_{h}, p_{h}\right)+\left(\nabla^{s} \delta \mathbf{v}_{h}, 2 \mu_{h} \nabla^{s} \mathbf{v}_{h}\right)+\left(\nabla^{s} \delta \mathbf{v}_{h}, 2 \mu_{h} \nabla^{s} \tilde{\mathbf{v}}\right)=F_{v}\left(\delta \mathbf{v}_{h}\right) \quad \forall \delta \mathbf{v}_{h} \\
& \left(\delta p_{h}, \nabla \cdot \mathbf{v}_{h}\right)+\left(\delta p_{h}, \nabla \cdot \tilde{\mathbf{v}}\right)=0 \quad \forall \delta p_{h} \\
& \left(\delta \vartheta_{h}, c_{h} \partial_{t} \vartheta_{h}\right)+\left(\delta \vartheta_{h}, c_{h}\left(\mathbf{v}_{h}+\tilde{\mathbf{v}}\right) \cdot \nabla \vartheta_{h}\right)+\left(\delta \vartheta_{h}, c_{h}\left(\mathbf{v}_{h}+\tilde{\mathbf{v}}\right) \cdot \nabla \tilde{\vartheta}\right) \\
& \quad+\left(\nabla \delta \vartheta_{h}, \kappa_{h} \nabla \vartheta_{h}\right)+\left(\nabla \delta \vartheta_{h}, \kappa_{h} \nabla \tilde{\vartheta}\right)=F_{\vartheta}\left(\delta \vartheta_{h}\right) \quad \forall \delta \vartheta_{h}
\end{aligned}
$$

The second system of equations represents the projection of the governing equations onto the corresponding infinite-dimensional sub-grid scale spaces, and is given by

$$
\begin{aligned}
& \left(\nabla \cdot \delta \tilde{\mathbf{v}}, p_{h}\right)+\left(\nabla^{s} \delta \tilde{\mathbf{v}}, 2 \mu_{h} \nabla^{s} \mathbf{v}_{h}\right)+\left(\nabla^{s} \delta \tilde{\mathbf{v}}, 2 \mu_{h} \nabla^{s} \tilde{\mathbf{v}}\right)=F_{v}(\delta \tilde{\mathbf{v}}) \quad \forall \delta \tilde{\mathbf{v}} \\
& \left(\delta \tilde{\vartheta}, c_{h} \partial_{t} \vartheta_{h}\right)+\left(\delta \tilde{\vartheta}, c_{h}\left(\mathbf{v}_{h}+\tilde{\mathbf{v}}\right) \cdot \nabla \vartheta_{h}\right)+\left(\delta \tilde{\vartheta}, c_{h}\left(\mathbf{v}_{h}+\tilde{\mathbf{v}}\right) \cdot \nabla \tilde{\vartheta}\right) \\
& \quad+\left(\nabla \delta \tilde{\vartheta}, \kappa_{h} \nabla \vartheta_{h}\right)+\left(\nabla \delta \tilde{\vartheta}, \kappa_{h} \nabla \tilde{\vartheta}\right)=F_{\vartheta}(\delta \tilde{\vartheta}) \quad \forall \delta \tilde{\vartheta}
\end{aligned}
$$

Note that modeling the gradients of the sub-grid scales is more involved than modeling the sub-grid scales themselves. Therefore integrating some terms by parts at the element level and taking into account that the sub-grid scales vanish at the inter-element boundaries, the first system of equations can be conveniently written as

$$
\begin{aligned}
& \left(\nabla \cdot \delta \mathbf{v}_{h}, p_{h}\right)+\left(\nabla^{s} \delta \mathbf{v}_{h}, 2 \mu_{h} \nabla^{s} \mathbf{v}_{h}\right)-\left(\nabla \cdot\left(2 \mu_{h} \nabla^{s} \delta \mathbf{v}_{h}\right), \tilde{\mathbf{v}}\right)=F_{v}\left(\delta \mathbf{v}_{h}\right) \quad \forall \delta \mathbf{v}_{h} \\
& \left(\delta p_{h}, \nabla \cdot \mathbf{v}_{h}\right)-\left(\nabla \delta p_{h}, \tilde{\mathbf{v}}\right)=0 \quad \forall \delta p_{h} \\
& \left(\delta \vartheta_{h}, c_{h} \partial_{t} \vartheta_{h}\right)+\left(\delta \vartheta_{h}, c_{h}\left(\mathbf{v}_{h}+\tilde{\mathbf{v}}\right) \cdot \nabla \vartheta_{h}\right)-\left(\nabla \cdot\left(c_{h}\left(\mathbf{v}_{h}+\tilde{\mathbf{v}}\right) \delta \vartheta_{h}\right), \tilde{\vartheta}\right) \\
& \quad+\left(\nabla \delta \vartheta_{h}, \kappa_{h} \nabla \vartheta_{h}\right)-\left(\nabla \cdot\left(\kappa_{h} \nabla \delta \vartheta_{h}\right), \tilde{\vartheta}\right)=F_{\vartheta}\left(\delta \vartheta_{h}\right) \quad \forall \delta \vartheta_{h}
\end{aligned}
$$

On the other hand, for the second system of equations it is convenient to write them in terms of the corresponding finite element residuals of the governing equations. Therefore integrating some terms by parts at the element level and taking into account that the sub-grid scales vanish at the inter-element boundaries, the second system of equations can be conveniently written as

$$
\begin{array}{rr}
-\left(\delta \tilde{\mathbf{v}}, \nabla \cdot\left(2 \mu_{h} \nabla^{s} \tilde{\mathbf{v}}\right)\right)=\left(\delta \tilde{\mathbf{v}}, \mathbf{r}_{v, h}\right) & \forall \delta \tilde{\mathbf{v}} \\
-\left(\delta \tilde{\vartheta}, c_{h}\left(\mathbf{v}_{h}+\tilde{\mathbf{v}}\right) \cdot \nabla \tilde{\vartheta}-\nabla \cdot\left(\kappa_{h} \nabla \tilde{\vartheta}\right)\right)=\left(\delta \tilde{\vartheta}, r_{\vartheta, h}\right) & \forall \delta \tilde{\vartheta}
\end{array}
$$

where the finite element residuals of the velocity and temperature equations, arising from the local governing equations, are defined as

$$
\begin{aligned}
& \mathbf{r}_{v, h}:=\nabla p_{h}+\nabla \cdot\left(2 \mu_{h} \nabla^{s} \mathbf{v}_{h}\right)+\mathbf{b}_{h} \\
& r_{\vartheta, h}:=c_{h} \partial_{t} \vartheta_{h}+c_{h}\left(\mathbf{v}_{h}+\tilde{\mathbf{v}}\right) \cdot \nabla \vartheta_{h}-\nabla \cdot\left(\kappa_{h} \nabla \vartheta_{h}\right)-r_{h}-\mathcal{D}_{h}
\end{aligned}
$$

Note that the sub-grid scale velocity appears in the convective term of the temperature residual. 
The idea now is to find an approximate solution for the second system of variational equations, finding an approximate discrete solution for the sub-grid scales within each element of the finite element partition. Once the approximation for the sub-grid scales has been found, they are substituted into the first system of variational equations, resulting in an enhanced stable mixed variational formulation of the IBVP.

If $\tilde{\mathcal{P}_{v}}$ and $\tilde{\mathcal{P}_{\vartheta}}$ denote the projections onto the sub-grid scale velocity and temperature spaces, respectively, the second system of equations can be written as

$$
\begin{array}{r}
-\tilde{\mathcal{P}_{v}}\left(\nabla \cdot\left(2 \mu_{h} \nabla^{s} \tilde{\mathbf{v}}\right)\right)=\tilde{\mathcal{P}}_{v}\left(\mathbf{r}_{v, h}\right) \\
-\tilde{\mathcal{P}_{\vartheta}}\left(c_{h}\left(\mathbf{v}_{h}+\tilde{\mathbf{v}}\right) \cdot \nabla \tilde{\vartheta}-\nabla \cdot\left(\kappa_{h} \nabla \tilde{\vartheta}\right)\right)=\tilde{\mathcal{P}}_{\vartheta}\left(r_{\vartheta, h}\right)
\end{array}
$$

These equations need to be solved within each element, at the element level, using homogeneous velocity and temperature Dirichlet boundary conditions.

Using the same arguments as in [39], now extended to thermally coupled problems, the sub-grid scales are approximated within each element of the finite element partition as

$$
\begin{aligned}
& \frac{1}{\tau_{v}} \tilde{\mathbf{v}}=\tilde{\mathcal{P}_{v}}\left(\mathbf{r}_{v, h}\right)=\tilde{\mathcal{P}_{v}}\left(\nabla p_{h}+\nabla \cdot\left(2 \mu_{h} \nabla^{s} \mathbf{v}_{h}\right)+\mathbf{b}_{h}\right) \\
& \frac{1}{\tau_{\vartheta}} \tilde{\vartheta}=-\tilde{\mathcal{P}_{\vartheta}}\left(r_{\vartheta, h}\right)=-\tilde{\mathcal{P}_{\vartheta}}\left(c_{h} \partial_{t} \vartheta_{h}+c_{h}\left(\mathbf{v}_{h}+\tilde{\mathbf{v}}\right) \cdot \nabla \vartheta_{h}-\nabla \cdot\left(\kappa_{h} \nabla \vartheta_{h}\right)-r_{h}-\mathcal{D}_{h}\right)
\end{aligned}
$$

where the stabilization parameters introduced above are approximated as

$$
\begin{aligned}
\tau_{v} & =\left(c_{1} \frac{2 \mu_{h}}{h^{2}}\right)^{-1}=\frac{1}{c_{1}} \frac{h^{2}}{2 \mu_{h}} \\
\tau_{\vartheta} & =\left(c_{1} \frac{\kappa_{h}}{h^{2}}+c_{2} \frac{c_{h}\left|\mathbf{v}_{h}+\tilde{\mathbf{v}}\right|}{h}\right)^{-1}
\end{aligned}
$$

where $h$ is the element size, and $c_{1}$ and $c_{2}$ are algorithmic constants.

Note that, taking into account that we have assumed that the material parameters and the dissipation rate were not dependent on the sub-grid scales, the resulting system of equations for the sub-grid scales is linear, the stabilization parameter for the velocity do not depends on the sub-grid scales, while the stabilization parameter for the temperature depends on the sub-grid scale velocity.

Introducing the stabilized velocity, pressure and temperature given by

$$
\begin{aligned}
\mathbf{v}_{h}^{\text {stab }} & :=\mathbf{v}_{h}+\tilde{\mathbf{v}}=\mathbf{v}_{h}+\tau_{v} \tilde{\mathcal{P}}_{v}\left(\nabla p_{h}+\nabla \cdot\left(2 \mu_{h} \nabla^{s} \mathbf{v}_{h}\right)+\mathbf{b}_{h}\right) \\
p_{h}^{\text {stab }} & :=p_{h} \\
\vartheta_{h}^{s t a b} & :=\vartheta_{h}+\tilde{\vartheta}=\vartheta_{h}-\tau_{\vartheta} \tilde{\mathcal{P}}_{\vartheta}\left(c_{h} \partial_{t} \vartheta_{h}+c_{h} \mathbf{v}_{h}^{\text {stab }} \cdot \nabla \vartheta_{h}-\nabla \cdot\left(\kappa_{h} \nabla \vartheta_{h}\right)-r_{h}-\mathcal{D}_{h}\right)
\end{aligned}
$$


the quasi-static sub-grid scale stabilized mixed variational formulation of the IBVP can be written as

$$
\begin{aligned}
& \left(\nabla \cdot \delta \mathbf{v}_{h}, p_{h}^{\text {stab }}\right)+\left(\nabla^{s} \delta \mathbf{v}_{h}, 2 \mu_{h} \nabla^{s} \mathbf{v}_{h}\right) \\
& \quad-\left(\nabla \cdot\left(2 \mu_{h} \nabla^{s} \delta \mathbf{v}_{h}\right), \mathbf{v}_{h}^{\text {stab }}-\mathbf{v}_{h}\right)=F_{v}\left(\delta \mathbf{v}_{h}\right) \quad \forall \delta \mathbf{v}_{h} \\
& \left(\delta p_{h}, \nabla \cdot \mathbf{v}_{h}\right)-\left(\nabla \delta p_{h}, \mathbf{v}_{h}^{\text {stab }}-\mathbf{v}_{h}\right)=0 \quad \forall \delta p_{h} \\
& \left(\delta \vartheta_{h}, c_{h} \partial_{t} \vartheta_{h}\right)+\left(\delta \vartheta_{h}, c_{h} \mathbf{v}_{h}^{\text {stab }} \cdot \nabla \vartheta_{h}\right)+\left(\nabla \delta \vartheta_{h}, \kappa_{h} \nabla \vartheta_{h}\right) \\
& \quad-\left(\nabla \cdot\left(c_{h} \mathbf{v}_{h}^{\text {stab }} \delta \vartheta_{h}\right), \vartheta_{h}^{\text {stab }}-\vartheta_{h}\right)-\left(\nabla \cdot\left(\kappa_{h} \nabla \delta \vartheta_{h}\right), \vartheta_{h}^{\text {stab }}-\vartheta_{h}\right)=F_{\vartheta}\left(\delta \vartheta_{h}\right) \quad \forall \delta \vartheta_{h}
\end{aligned}
$$

Taking into account that $\mathbf{v}_{h}^{\text {stab }}:=\mathbf{v}_{h}+\tilde{\mathbf{v}}$ is divergence free, the following expression holds

$$
\begin{aligned}
\nabla \cdot\left(c_{h} \mathbf{v}_{h}^{\text {stab }} \delta \vartheta_{h}\right) & =\nabla c_{h} \cdot \mathbf{v}_{h}^{\text {stab }} \delta \vartheta_{h}+c_{h} \delta \vartheta_{h} \nabla \cdot \mathbf{v}_{h}^{\text {stab }}+c_{h} \mathbf{v}_{h}^{\text {stab }} \cdot \nabla \delta \vartheta_{h} \\
& =\nabla c_{h} \cdot \mathbf{v}_{h}^{\text {stab }} \delta \vartheta_{h}+c_{h} \mathbf{v}_{h}^{\text {stab }} \cdot \nabla \delta \vartheta_{h}
\end{aligned}
$$

and the quasi-static sub-grid scale stabilized mixed variational formulation of the IBVP can be written as

$$
\begin{aligned}
& \left(\nabla \cdot \delta \mathbf{v}_{h}, p_{h}^{\text {stab }}\right)+\left(\nabla^{s} \delta \mathbf{v}_{h}, 2 \mu_{h} \nabla^{s} \mathbf{v}_{h}\right) \\
& -\left(\nabla \cdot\left(2 \mu_{h} \nabla^{s} \delta \mathbf{v}_{h}\right), \mathbf{v}_{h}^{\text {stab }}-\mathbf{v}_{h}\right)=F_{v}\left(\delta \mathbf{v}_{h}\right) \quad \forall \delta \mathbf{v}_{h} \\
& \left(\delta p_{h}, \nabla \cdot \mathbf{v}_{h}\right)-\left(\nabla \delta p_{h}, \mathbf{v}_{h}^{\text {stab }}-\mathbf{v}_{h}\right)=0 \quad \forall \delta p_{h} \\
& \left(\delta \vartheta_{h}, c_{h} \partial_{t} \vartheta_{h}\right)+\left(\delta \vartheta_{h}, c_{h} \mathbf{v}_{h}^{\text {stab }} \cdot \nabla \vartheta_{h}\right)+\left(\nabla \delta \vartheta_{h}, \kappa_{h} \nabla \vartheta_{h}\right) \\
& \quad-\left(c_{h} \mathbf{v}_{h}^{\text {stab }} \cdot \nabla \delta \vartheta_{h}, \vartheta_{h}^{\text {stab }}-\vartheta_{h}\right)-\left(\nabla c_{h} \cdot \mathbf{v}_{h}^{\text {stab }} \delta \vartheta_{h}, \vartheta_{h}^{\text {stab }}-\vartheta_{h}\right) \\
& -\left(\nabla \cdot\left(\kappa_{h} \nabla \delta \vartheta_{h}\right), \vartheta_{h}^{\text {stab }}-\vartheta_{h}\right)=F_{\vartheta}\left(\delta \vartheta_{h}\right) \quad \forall \delta \vartheta_{h}
\end{aligned}
$$

\subsubsection{P1/P1/P1 linear velocity/linear pressure/linear temperature elements}

For P1/P1/P1 linear velocity/linear pressure/linear temperature elements, the terms $\nabla^{s} \mathbf{v}_{h}$ and $\nabla \vartheta_{h}$, as well as $\nabla^{s} \delta \mathbf{v}_{h}$ and $\nabla \delta \vartheta_{h}$, are constants within an element and the following expressions hold

$$
\begin{array}{ll}
\nabla \cdot\left(\nabla^{s} \mathbf{v}_{h}\right)=0, & \nabla \cdot\left(\nabla^{s} \delta \mathbf{v}_{h}\right)=0, \\
\nabla \cdot\left(\nabla \vartheta_{h}\right)=0, & \nabla \cdot\left(\nabla \delta \vartheta_{h}\right)=0
\end{array}
$$

On the other hand, note that the equivalent viscosity is a highly non-linear function of the equivalent strain rate, which is a function of $\nabla^{s} \mathbf{v}_{h}$, and, in general, of the temperature. Note that for $\mathrm{P} 1 / \mathrm{P} 1 / \mathrm{P} 1$ linear velocity/linear pressure/linear temperature elements, the equivalent strain rate is constant within an element. Then, for $\mathrm{P} 1 / \mathrm{P} 1 / \mathrm{P} 1$ linear velocity/linear pressure/linear temperature elements, the equivalent viscosity will be constant within an element 
only if it is not a function of the temperature or if being a function of the temperature, the temperature is constant within the element.

Then, if the equivalent viscosity and the thermal conductivity are temperature dependent, the following expressions hold for P1/P1/P1 linear velocity/linear pressure/linear temperature elements

$$
\begin{aligned}
\nabla \cdot\left(2 \mu_{h} \nabla^{s} \mathbf{v}_{h}\right) & =2 \mu_{h} \nabla \cdot \nabla^{s} \mathbf{v}_{h}+2 \nabla^{s} \mathbf{v}_{h} \nabla \mu_{h}=2 \nabla^{s} \mathbf{v}_{h} \nabla \mu_{h}, \\
\nabla \cdot\left(2 \mu_{h} \nabla^{s} \delta \mathbf{v}_{h}\right) & =2 \mu_{h} \nabla \cdot \nabla^{s} \delta \mathbf{v}_{h}+2 \nabla^{s} \delta \mathbf{v}_{h} \nabla \mu_{h}=2 \nabla^{s} \delta \mathbf{v}_{h} \nabla \mu_{h}, \\
\nabla \cdot\left(\kappa_{h} \nabla \vartheta_{h}\right) & =\kappa_{h} \nabla \cdot \nabla \vartheta_{h}+\nabla \kappa_{h} \cdot \nabla \vartheta_{h}=\nabla \kappa_{h} \cdot \nabla \vartheta_{h}, \\
\nabla \cdot\left(\kappa_{h} \nabla \delta \vartheta_{h}\right) & =\kappa_{h} \nabla \cdot \nabla \delta \vartheta_{h}+\nabla \kappa_{h} \cdot \nabla \delta \vartheta_{h}=\nabla \kappa_{h} \cdot \nabla \delta \vartheta_{h}
\end{aligned}
$$

The quasi-static sub-grid scales for $\mathrm{P} 1 / \mathrm{P} 1 / \mathrm{P} 1$ linear velocity/linear pressure/linear temperature elements are given by,

$$
\begin{aligned}
& \frac{1}{\tau_{v}} \tilde{\mathbf{v}}=\tilde{\mathcal{P}_{v}}\left(\mathbf{r}_{v, h}\right)=\tilde{\mathcal{P}_{v}}\left(\nabla p_{h}+2 \nabla^{s} \mathbf{v}_{h} \nabla \mu_{h}+\mathbf{b}_{h}\right) \\
& \frac{1}{\tau_{\vartheta}} \tilde{\vartheta}=-\tilde{\mathcal{P}_{\vartheta}}\left(r_{\vartheta, h}\right)=-\tilde{\mathcal{P}_{\vartheta}}\left(c_{h} \partial_{t} \vartheta_{h}+c_{h}\left(\mathbf{v}_{h}+\tilde{\mathbf{v}}\right) \cdot \nabla \vartheta_{h}-\nabla \kappa_{h} \cdot \nabla \vartheta_{h}-r_{h}-\mathcal{D}_{h}\right)
\end{aligned}
$$

The stabilized velocity, pressure and temperature for $\mathrm{P} 1 / \mathrm{P} 1 / \mathrm{P} 1$ linear velocity/linear pressure/linear temperature elements, take the form

$$
\begin{aligned}
& \mathbf{v}_{h}^{\text {stab }}:=\mathbf{v}_{h}+\tilde{\mathbf{v}}=\mathbf{v}_{h}+\tau_{v} \tilde{\mathcal{P}_{v}}\left(\nabla p_{h}+2 \nabla^{s} \mathbf{v}_{h} \nabla \mu_{h}+\mathbf{b}_{h}\right) \\
& p_{h}^{\text {stab }}:=p_{h} \\
& \vartheta_{h}^{\text {stab }}:=\vartheta_{h}+\tilde{\vartheta}=\vartheta_{h}-\tau_{\vartheta} \tilde{\mathcal{P}}_{\vartheta}\left(c_{h} \partial_{t} \vartheta_{h}+c_{h} \mathbf{v}_{h}^{\text {stab }} \cdot \nabla \vartheta_{h}-\nabla \kappa_{h} \cdot \nabla \vartheta_{h}-r_{h}-\mathcal{D}_{h}\right)
\end{aligned}
$$

The quasi-static sub-grid scale stabilized mixed variational formulation for $\mathrm{P} 1 / \mathrm{P} 1 / \mathrm{P} 1$ linear velocity/linear pressure/linear temperature elements, reads

$$
\begin{aligned}
& \left(\nabla \cdot \delta \mathbf{v}_{h}, p_{h}^{\text {stab }}\right)+\left(\nabla^{s} \delta \mathbf{v}_{h}, 2 \mu_{h} \nabla^{s} \mathbf{v}_{h}\right) \\
& \quad-\left(2 \nabla^{s} \delta \mathbf{v}_{h} \nabla \mu_{h}, \mathbf{v}_{h}^{\text {stab }}-\mathbf{v}_{h}\right)=F_{v}\left(\delta \mathbf{v}_{h}\right) \quad \forall \delta \mathbf{v}_{h} \\
& \left(\delta p_{h}, \nabla \cdot \mathbf{v}_{h}\right)-\left(\nabla \delta p_{h}, \mathbf{v}_{h}^{s t a b}-\mathbf{v}_{h}\right)=0 \quad \forall \delta p_{h} \\
& \left(\delta \vartheta_{h}, c_{h} \partial_{t} \vartheta_{h}\right)+\left(\delta \vartheta_{h}, c_{h} \mathbf{v}_{h}^{\text {stab }} \cdot \nabla \vartheta_{h}\right)+\left(\nabla \delta \vartheta_{h}, \kappa_{h} \nabla \vartheta_{h}\right) \\
& \quad-\left(c_{h} \mathbf{v}_{h}^{s t a b} \cdot \nabla \delta \vartheta_{h}, \vartheta_{h}^{s t a b}-\vartheta_{h}\right)-\left(\nabla c_{h} \cdot \mathbf{v}_{h}^{\text {stab }} \delta \vartheta_{h}, \vartheta_{h}^{\text {stab }}-\vartheta_{h}\right) \\
& \quad-\left(\nabla \kappa_{h} \cdot \nabla \delta \vartheta_{h}, \vartheta_{h}^{\text {stab }}-\vartheta_{h}\right)=F_{\vartheta}\left(\delta \vartheta_{h}\right) \quad \forall \delta \vartheta_{h}
\end{aligned}
$$

REMARK 1. Temperature independent equivalent viscosity, constant thermal conductivity and constant heat capacity. Let us consider now P1/P1/P1 linear velocity/linear pressure/linear temperature elements with temperature independent equivalent viscosity, constant thermal conductivity, and constant heat capacity. Note that the equivalent viscosity is a function of the 
equivalent strain rate, which is a function of the symmetric spatial gradient of the velocity, and the temperature. Using a linear interpolation for the velocity and the temperature, the spatial gradient of the velocity is constant within an element, and its spatial gradient will be equal to zero if the viscosity is temperature independent. Then, if the equivalent viscosity is temperature independent, the thermal conductivity is constant, and the heat capacity is constant, the following expressions hold at the element level

$$
\nabla \mu_{h}=\mathbf{0}, \quad \nabla \kappa_{h}=\mathbf{0}, \quad \nabla c_{h}=\mathbf{0}
$$

The quasi-static sub-grid scales for $\mathrm{P} 1 / \mathrm{P} 1 / \mathrm{P} 1$ linear velocity/linear pressure/linear temperature elements, temperature independent equivalent viscosity, and constant thermal conductivity, take the form

$$
\begin{aligned}
& \frac{1}{\tau_{v}} \tilde{\mathbf{v}}=\tilde{\mathcal{P}_{v}}\left(\mathbf{r}_{v, h}\right)=\tilde{\mathcal{P}_{v}}\left(\nabla p_{h}+\mathbf{b}_{h}\right) \\
& \frac{1}{\tau_{\vartheta}} \tilde{\vartheta}=-\tilde{\mathcal{P}_{\vartheta}}\left(r_{\vartheta, h}\right)=-\tilde{\mathcal{P}_{\vartheta}}\left(c_{h} \partial_{t} \vartheta_{h}+c_{h}\left(\mathbf{v}_{h}+\tilde{\mathbf{v}}\right) \cdot \nabla \vartheta_{h}-r_{h}-\mathcal{D}_{h}\right)
\end{aligned}
$$

and the stabilized velocity, pressure and temperature, take the form

$$
\begin{aligned}
\mathbf{v}_{h}^{\text {stab }} & :=\mathbf{v}_{h}+\tilde{\mathbf{v}}=\mathbf{v}_{h}+\tau_{v} \tilde{\mathcal{P}_{v}}\left(\nabla p_{h}+\mathbf{b}_{h}\right) \\
p_{h}^{\text {stab }} & :=p_{h} \\
\vartheta_{h}^{\text {stab }} & :=\vartheta_{h}+\tilde{\vartheta}=\vartheta_{h}-\tau_{\vartheta} \tilde{\mathcal{P}_{\vartheta}}\left(c_{h} \partial_{t} \vartheta_{h}+c_{h} \mathbf{v}_{h}^{\text {stab }} \cdot \nabla \vartheta_{h}-r_{h}-\mathcal{D}_{h}\right)
\end{aligned}
$$

The quasi-static sub-grid scale stabilized mixed variational formulation for $\mathrm{P} 1 / \mathrm{P} 1 / \mathrm{P} 1$ linear velocity/linear pressure/linear temperature elements, temperature independent equivalent viscosity, constant thermal conductivity, and constant heat capacity, reads

$$
\begin{aligned}
& \left(\nabla \cdot \delta \mathbf{v}_{h}, p_{h}^{\text {stab }}\right)+\left(\nabla^{s} \delta \mathbf{v}_{h}, 2 \mu_{h} \nabla^{s} \mathbf{v}_{h}\right)=F_{v}\left(\delta \mathbf{v}_{h}\right) \quad \forall \delta \mathbf{v}_{h} \\
& \left(\delta p_{h}, \nabla \cdot \mathbf{v}_{h}\right)-\left(\nabla \delta p_{h}, \mathbf{v}_{h}^{\text {stab }}-\mathbf{v}_{h}\right)=0 \quad \forall \delta p_{h} \\
& \left(\delta \vartheta_{h}, c_{h} \partial_{t} \vartheta_{h}\right)+\left(\delta \vartheta_{h}, c_{h} \mathbf{v}_{h}^{\text {stab }} \cdot \nabla \vartheta_{h}\right)+\left(\nabla \delta \vartheta_{h}, \kappa_{h} \nabla \vartheta_{h}\right) \\
& \quad-\left(c_{h} \mathbf{v}_{h}^{\text {stab }} \cdot \nabla \delta \vartheta_{h}, \vartheta_{h}^{\text {stab }}-\vartheta_{h}\right)=F_{\vartheta}\left(\delta \vartheta_{h}\right) \quad \forall \delta \vartheta_{h}
\end{aligned}
$$

Substituting the quasi-static sub-grid scales into the above expression, the sub-grid scale stabilized mixed variational formulation reads 


$$
\begin{aligned}
& \left(\nabla \cdot \delta \mathbf{v}_{h}, p_{h}\right)+\left(\nabla^{s} \delta \mathbf{v}_{h}, 2 \mu_{h} \nabla^{s} \mathbf{v}_{h}\right)=F_{v}\left(\delta \mathbf{v}_{h}\right) \quad \forall \delta \mathbf{v}_{h} \\
& \left(\delta p_{h}, \nabla \cdot \mathbf{v}_{h}\right) \\
& \quad-\tau_{v}\left(\nabla \delta p_{h}, \tilde{\mathcal{P}_{v}}\left(\nabla p_{h}\right)\right) \\
& \quad-\tau_{v}\left(\nabla \delta p_{h}, \tilde{\mathcal{P}_{v}}\left(\mathbf{b}_{h}\right)\right)=0 \quad \forall \delta p_{h} \\
& \left(\delta \vartheta_{h}, c_{h} \partial_{t} \vartheta_{h}\right)+\left(\delta \vartheta_{h}, c_{h} \mathbf{v}_{h}^{\text {stab }} \cdot \nabla \vartheta_{h}\right)+\left(\nabla \delta \vartheta_{h}, \kappa_{h} \nabla \vartheta_{h}\right) \\
& \quad+\tau_{\vartheta}\left(c_{h} \mathbf{v}_{h}^{\text {stab }} \cdot \nabla \delta \vartheta_{h}, \tilde{\mathcal{P}_{\vartheta}}\left(c_{h} \partial_{t} \vartheta_{h}\right)\right) \\
& \quad+\tau_{\vartheta}\left(c_{h} \mathbf{v}_{h}^{\text {stab }} \cdot \nabla \delta \vartheta_{h}, \tilde{\mathcal{P}_{\vartheta}}\left(c_{h} \mathbf{v}_{h}^{\text {stab }} \cdot \nabla \vartheta_{h}\right)\right) \\
& \quad-\tau_{\vartheta}\left(c_{h} \mathbf{v}_{h}^{\text {stab }} \cdot \nabla \delta \vartheta_{h}, \tilde{\mathcal{P}_{\vartheta}}\left(r_{h}\right)\right) \\
& \quad-\tau_{\vartheta}\left(c_{h} \mathbf{v}_{h}^{\text {stab }} \cdot \nabla \delta \vartheta_{h}, \tilde{\mathcal{P}_{\vartheta}}\left(\mathcal{D}_{h}\right)\right)=F_{\vartheta}\left(\delta \vartheta_{h}\right) \quad \forall \delta \vartheta_{h}
\end{aligned}
$$

\subsubsection{Quasi-static Algebraic Sub-grid Scale (ASGS)}

For the Algebraic Sub-grid Scale (ASGS) method, the projections onto the sub-grid scale spaces velocity and temperature spaces are defined as $\tilde{\mathcal{P}_{v}}=I$ and $\tilde{\mathcal{P}_{\vartheta}}=I$, respectively, yielding

$$
\begin{aligned}
& \frac{1}{\tau_{v}} \tilde{\mathbf{v}}=\tilde{\mathcal{P}_{v}}\left(\mathbf{r}_{v, h}\right)=\mathbf{r}_{v, h}=\nabla p_{h}+2 \nabla^{s} \mathbf{v}_{h} \nabla \mu_{h}+\mathbf{b}_{h} \\
& \frac{1}{\tau_{\vartheta}} \tilde{\vartheta}=-\tilde{\mathcal{P}_{\vartheta}}\left(r_{\vartheta, h}\right)=-r_{\vartheta, h}=-\left(c_{h} \partial_{t} \vartheta_{h}+c_{h} \mathbf{v}_{h}^{\text {stab }} \cdot \nabla \vartheta_{h}-\nabla \kappa_{h} \cdot \nabla \vartheta_{h}-r_{h}-\mathcal{D}_{h}\right)
\end{aligned}
$$

The ASGS stabilized velocity, pressure and temperature for $\mathrm{P} 1 / \mathrm{P} 1 / \mathrm{P} 1$ linear velocity/linear pressure/linear temperature elements take the form

$$
\begin{aligned}
& \mathbf{v}_{h}^{\text {stab }}:=\mathbf{v}_{h}+\tilde{\mathbf{v}}=\mathbf{v}_{h}+\tau_{v}\left(\nabla p_{h}+2 \nabla^{s} \mathbf{v}_{h} \nabla \mu_{h}+\mathbf{b}_{h}\right) \\
& p_{h}^{\text {stab }}:=p_{h} \\
& \vartheta_{h}^{\text {stab }}:=\vartheta_{h}+\tilde{\vartheta}=\vartheta_{h}-\tau_{\vartheta}\left(c_{h} \partial_{t} \vartheta_{h}+c_{h} \mathbf{v}_{h}^{\text {stab }} \cdot \nabla \vartheta_{h}-\nabla \kappa_{h} \cdot \nabla \vartheta_{h}-r_{h}-\mathcal{D}_{h}\right)
\end{aligned}
$$

REMARK 2. Temperature independent equivalent viscosity, constant thermal conductivity and constant heat capacity. The quasi-static ASGS sub-grid scales for P1/P1/P1 linear velocity/linear pressure/linear temperature elements, with temperature independent equivalent viscosity, and constant thermal conductivity, take the form

$$
\begin{aligned}
& \frac{1}{\tau_{v}} \tilde{\mathbf{v}}=\tilde{\mathcal{P}}_{v}\left(\mathbf{r}_{v, h}\right)=\mathbf{r}_{v, h}=\nabla p_{h}+\mathbf{b}_{h} \\
& \frac{1}{\tau_{\vartheta}} \tilde{\vartheta}=-\tilde{\mathcal{P}}_{\vartheta}\left(r_{\vartheta, h}\right)=-r_{\vartheta, h}=-\left(c_{h} \partial_{t} \vartheta_{h}+c_{h} \mathbf{v}_{h}^{s t a b} \cdot \nabla \vartheta_{h}-r_{h}-\mathcal{D}_{h}\right)
\end{aligned}
$$

and the ASGS stabilized velocity, pressure and temperature take the form 


$$
\begin{aligned}
& \mathbf{v}_{h}^{\text {stab }}:=\mathbf{v}_{h}+\tilde{\mathbf{v}}=\mathbf{v}_{h}+\tau_{v}\left(\nabla p_{h}+\mathbf{b}_{h}\right) \\
& p_{h}^{\text {stab }}:=p_{h} \\
& \vartheta_{h}^{\text {stab }}:=\vartheta_{h}+\tilde{\vartheta}=\vartheta_{h}-\tau_{\vartheta}\left(c_{h} \partial_{t} \vartheta_{h}+c_{h} \mathbf{v}_{h}^{\text {stab }} \cdot \nabla \vartheta_{h}-r_{h}-\mathcal{D}_{h}\right)
\end{aligned}
$$

The quasi-static ASGS sub-grid scale stabilized mixed variational formulation reads

$$
\begin{aligned}
& \left(\nabla \cdot \delta \mathbf{v}_{h}, p_{h}\right)+\left(\nabla^{s} \delta \mathbf{v}_{h}, 2 \mu_{h} \nabla^{s} \mathbf{v}_{h}\right)=F_{v}\left(\delta \mathbf{v}_{h}\right) \quad \forall \delta \mathbf{v}_{h} \\
& \left(\delta p_{h}, \nabla \cdot \mathbf{v}_{h}\right) \\
& \quad-\tau_{v}\left(\nabla \delta p_{h}, \nabla p_{h}\right) \\
& \quad-\tau_{v}\left(\nabla \delta p_{h}, \mathbf{b}_{h}\right)=0 \quad \forall \delta p_{h} \\
& \left(\delta \vartheta_{h}, c_{h} \partial_{t} \vartheta_{h}\right)+\left(\delta \vartheta_{h}, c_{h} \mathbf{v}_{h}^{\text {stab }} \cdot \nabla \vartheta_{h}\right)+\left(\nabla \delta \vartheta_{h}, \kappa_{h} \nabla \vartheta_{h}\right) \\
& \quad+\tau_{\vartheta}\left(c_{h} \mathbf{v}_{h}^{\text {stab }} \cdot \nabla \delta \vartheta_{h}, c_{h} \partial_{t} \vartheta_{h}\right) \\
& \quad+\tau_{\vartheta}\left(c_{h} \mathbf{v}_{h}^{\text {stab }} \cdot \nabla \delta \vartheta_{h}, c_{h} \mathbf{v}_{h}^{\text {stab }} \cdot \nabla \vartheta_{h}\right) \\
& \quad-\tau_{\vartheta}\left(c_{h} \mathbf{v}_{h}^{\text {stab }} \cdot \nabla \delta \vartheta_{h}, r_{h}\right) \\
& \quad-\tau_{\vartheta}\left(c_{h} \mathbf{v}_{h}^{\text {stab }} \cdot \nabla \delta \vartheta_{h}, \mathcal{D}_{h}\right)=F_{\vartheta}\left(\delta \vartheta_{h}\right) \quad \forall \delta \vartheta_{h}
\end{aligned}
$$

REMARK 3. Use of the finite element velocity, instead of the stabilized one, in the convective term of the temperature equation. Setting $\tau_{v}=0$ in the stabilized variational form of the energy balance equation, i.e. setting $\mathbf{v}_{h}^{\text {stab }}=\mathbf{v}_{h}$ in the convective term of the sub-grid scale temperature, the stabilized velocity, pressure and temperature read

$$
\begin{aligned}
& \mathbf{v}_{h}^{\text {stab }}:=\mathbf{v}_{h}+\tilde{\mathbf{v}}=\mathbf{v}_{h}+\tau_{v}\left(\nabla p_{h}+\mathbf{b}_{h}\right) \\
& p_{h}^{\text {stab }}:=p_{h} \\
& \vartheta_{h}^{\text {stab }}:=\vartheta_{h}+\tilde{\vartheta}=\vartheta_{h}-\tau_{\vartheta}\left(c_{h} \partial_{t} \vartheta_{h}+c_{h} \mathbf{v}_{h} \cdot \nabla \vartheta_{h}-r_{h}-\mathcal{D}_{h}\right)
\end{aligned}
$$

The quasi-static ASGS sub-grid scale stabilized mixed variational formulation reads

$$
\begin{aligned}
& \left(\nabla \cdot \delta \mathbf{v}_{h}, p_{h}\right)+\left(\nabla^{s} \delta \mathbf{v}_{h}, 2 \mu_{h} \nabla^{s} \mathbf{v}_{h}\right)=F_{v}\left(\delta \mathbf{v}_{h}\right) \quad \forall \delta \mathbf{v}_{h} \\
& \left(\delta p_{h}, \nabla \cdot \mathbf{v}_{h}\right) \\
& \quad-\tau_{v}\left(\nabla \delta p_{h}, \nabla p_{h}\right) \\
& \quad-\tau_{v}\left(\nabla \delta p_{h}, \mathbf{b}_{h}\right)=0 \quad \forall \delta p_{h} \\
& \left(\delta \vartheta_{h}, c_{h} \partial_{t} \vartheta_{h}\right)+\left(\delta \vartheta_{h}, c_{h} \mathbf{v}_{h} \cdot \nabla \vartheta_{h}\right)+\left(\nabla \delta \vartheta_{h}, \kappa_{h} \nabla \vartheta_{h}\right) \\
& \quad+\tau_{\vartheta}\left(c_{h} \mathbf{v}_{h} \cdot \nabla \delta \vartheta_{h}, c_{h} \partial_{t} \vartheta_{h}\right) \\
& \quad+\tau_{\vartheta}\left(c_{h} \mathbf{v}_{h} \cdot \nabla \delta \vartheta_{h}, c_{h} \mathbf{v}_{h} \cdot \nabla \vartheta_{h}\right) \\
& \quad-\tau_{\vartheta}\left(c_{h} \mathbf{v}_{h} \cdot \nabla \delta \vartheta_{h}, r_{h}\right) \\
& \quad-\tau_{\vartheta}\left(c_{h} \mathbf{v}_{h} \cdot \nabla \delta \vartheta_{h}, \mathcal{D}_{h}\right)=F_{\vartheta}\left(\delta \vartheta_{h}\right) \quad \forall \delta \vartheta_{h}
\end{aligned}
$$


REMARK 4. Classical GLS/SUPG stabilization method. Classical GLS pressure stabilization and SUPG convection stabilization methods can be recovered as a particular case of the ASGS stabilization method neglecting the following terms:

$$
\begin{array}{r}
\tau_{v}\left(\nabla \delta p_{h}, \mathbf{b}_{h}\right)=0 \\
\tau_{\vartheta}\left(c_{h} \mathbf{v}_{h} \cdot \nabla \delta \vartheta_{h}, c_{h} \partial_{t} \vartheta_{h}\right)=0 \\
\tau_{\vartheta}\left(c_{h} \mathbf{v}_{h} \cdot \nabla \delta \vartheta_{h}, r_{h}\right)=0 \\
\tau_{\vartheta}\left(c_{h} \mathbf{v}_{h} \cdot \nabla \delta \vartheta_{h}, \mathcal{D}_{h}\right)=0
\end{array}
$$

The quasi-static ASGS sub-grid scale stabilized mixed variational formulation corresponds to the classical GLS/SUPG stabilized mixed variational formulation and reads

$$
\begin{aligned}
& \left(\nabla \cdot \delta \mathbf{v}_{h}, p_{h}\right)+\left(\nabla^{s} \delta \mathbf{v}_{h}, 2 \mu_{h} \nabla^{s} \mathbf{v}_{h}\right)=F_{v}\left(\delta \mathbf{v}_{h}\right) \quad \forall \delta \mathbf{v}_{h} \\
& \left(\delta p_{h}, \nabla \cdot \mathbf{v}_{h}\right) \\
& \quad-\tau_{v}\left(\nabla \delta p_{h}, \nabla p_{h}\right)=0 \quad \forall \delta p_{h} \\
& \left(\delta \vartheta_{h}, c_{h} \partial_{t} \vartheta_{h}\right)+\left(\delta \vartheta_{h}, c_{h} \mathbf{v}_{h} \cdot \nabla \vartheta_{h}\right)+\left(\nabla \delta \vartheta_{h}, \kappa_{h} \nabla \vartheta_{h}\right) \\
& \quad+\tau_{\vartheta}\left(c_{h} \mathbf{v}_{h} \cdot \nabla \delta \vartheta_{h}, c_{h} \mathbf{v}_{h} \cdot \nabla \vartheta_{h}\right)=F_{\vartheta}\left(\delta \vartheta_{h}\right) \quad \forall \delta \vartheta_{h}
\end{aligned}
$$

\subsubsection{Quasi-static Orthogonal Sub-grid Scale (OSGS)}

For the Orthogonal Sub-grid Scale (OSGS) method, the projections onto the sub-grid scale velocity and temperature spaces are defined as $\tilde{\mathcal{P}_{v}}=\mathcal{P}_{h}^{\perp}$ and $\tilde{\mathcal{P}_{\vartheta}}=\mathcal{P}_{h}^{\perp}$, where $\mathcal{P}_{h}^{\perp}=I-\mathcal{P}_{h}$ represents the $L^{2}$ orthogonal projection, i.e. the $L^{2}$ projection onto the space which is orthogonal to the appropriate finite element one, and $\mathcal{P}_{h}$ represents the $L^{2}$ projection onto the appropriate, velocity or temperature, finite element space. The sub-grid scales turn out to be orthogonal to this finite element space.

Note that, assuming constant heat capacity, the orthogonal projection of the transient thermal term is zero, yielding

$$
\mathcal{P}_{h}^{\perp}\left(c_{h} \partial_{t} \vartheta_{h}\right)=0
$$

The quasi-static OSGS sub-grid scales for P1/P1/P1 linear velocity/linear pressure/linear temperature elements take the form

$$
\begin{aligned}
& \frac{1}{\tau_{v}} \tilde{\mathbf{v}}=\tilde{\mathcal{P}}_{v}\left(\mathbf{r}_{v, h}\right)=\mathcal{P}_{h}^{\perp}\left(\mathbf{r}_{v, h}\right)=\mathcal{P}_{h}^{\perp}\left(\nabla p_{h}+2 \nabla^{s} \mathbf{v}_{h} \nabla \mu_{h}+\mathbf{b}_{h}\right) \\
& \frac{1}{\tau_{\vartheta}} \tilde{\vartheta}=-\tilde{\mathcal{P}_{\vartheta}}\left(r_{\vartheta, h}\right)=-\mathcal{P}_{h}^{\perp}\left(r_{\vartheta, h}\right)=-\mathcal{P}_{h}^{\perp}\left(c_{h}\left(\mathbf{v}_{h}+\tilde{\mathbf{v}}\right) \cdot \nabla \vartheta_{h}-\nabla \kappa_{h} \cdot \nabla \vartheta_{h}-r_{h}-\mathcal{D}_{h}\right)
\end{aligned}
$$

The OSGS stabilized velocity, pressure and temperature for $\mathrm{P} 1 / \mathrm{P} 1 / \mathrm{P} 1$ linear velocity/linear pressure/linear temperature elements, take the form 


$$
\begin{aligned}
\mathbf{v}_{h}^{\text {stab }} & :=\mathbf{v}_{h}+\tilde{\mathbf{v}}=\mathbf{v}_{h}+\tau_{v} \mathcal{P}_{h}^{\perp}\left(\nabla p_{h}+2 \nabla^{s} \mathbf{v}_{h} \nabla \mu_{h}+\mathbf{b}_{h}\right) \\
p_{h}^{\text {stab }} & :=p_{h} \\
\vartheta_{h}^{\text {stab }} & :=\vartheta_{h}+\tilde{\vartheta}=\vartheta_{h}-\tau_{\vartheta} \mathcal{P}_{h}^{\perp}\left(c_{h}\left(\mathbf{v}_{h}+\tilde{\mathbf{v}}\right) \cdot \nabla \vartheta_{h}-\nabla \kappa_{h} \cdot \nabla \vartheta_{h}-r_{h}-\mathcal{D}_{h}\right)
\end{aligned}
$$

REMARK 5. Temperature independent equivalent viscosity, constant thermal conductivity and constant heat capacity. The quasi-static OSGS sub-grid scales for P1/P1/P1 linear velocity/linear pressure/linear temperature elements, with temperature independent equivalent viscosity, and constant thermal conductivity, take the form

$$
\begin{aligned}
& \frac{1}{\tau_{v}} \tilde{\mathbf{v}}=\tilde{\mathcal{P}}_{v}\left(\mathbf{r}_{v, h}\right)=\mathcal{P}_{h}^{\perp}\left(\mathbf{r}_{v, h}\right)=\mathcal{P}_{h}^{\perp}\left(\nabla p_{h}+\mathbf{b}_{h}\right) \\
& \frac{1}{\tau_{\vartheta}} \tilde{\vartheta}=-\tilde{\mathcal{P}_{\vartheta}}\left(r_{\vartheta, h}\right)=-\mathcal{P}_{h}^{\perp}\left(r_{\vartheta, h}\right)=-\mathcal{P}_{h}^{\perp}\left(c_{h}\left(\mathbf{v}_{h}+\tilde{\mathbf{v}}\right) \cdot \nabla \vartheta_{h}-r_{h}-\mathcal{D}_{h}\right)
\end{aligned}
$$

and the OSGS stabilized velocity, pressure and temperature take the form

$$
\begin{aligned}
& \mathbf{v}_{h}^{\text {stab }}:=\mathbf{v}_{h}+\tilde{\mathbf{v}}=\mathbf{v}_{h}+\tau_{v} \mathcal{P}_{h}^{\perp}\left(\nabla p_{h}+\mathbf{b}_{h}\right) \\
& p_{h}^{\text {stab }}:=p_{h} \\
& \vartheta_{h}^{\text {stab }}:=\vartheta_{h}+\tilde{\vartheta}=\vartheta_{h}-\tau_{\vartheta} \mathcal{P}_{h}^{\perp}\left(c_{h} \mathbf{v}_{h}^{\text {stab }} \cdot \nabla \vartheta_{h}-r_{h}-\mathcal{D}_{h}\right)
\end{aligned}
$$

The quasi-static OSGS sub-grid scale stabilized mixed variational formulation reads

$$
\begin{aligned}
& \left(\nabla \cdot \delta \mathbf{v}_{h}, p_{h}\right)+\left(\nabla^{s} \delta \mathbf{v}_{h}, 2 \mu_{h} \nabla^{s} \mathbf{v}_{h}\right)=F_{v}\left(\delta \mathbf{v}_{h}\right) \quad \forall \delta \mathbf{v}_{h} \\
& \left(\delta p_{h}, \nabla \cdot \mathbf{v}_{h}\right) \\
& \quad-\tau_{v}\left(\nabla \delta p_{h}, \mathcal{P}_{h}^{\perp}\left(\nabla p_{h}\right)\right) \\
& \quad-\tau_{v}\left(\nabla \delta p_{h}, \mathcal{P}_{h}^{\perp}\left(\mathbf{b}_{h}\right)\right)=0 \quad \forall \delta p_{h} \\
& \left(\delta \vartheta_{h}, c_{h} \partial_{t} \vartheta_{h}\right)+\left(\delta \vartheta_{h}, c_{h} \mathbf{v}_{h}^{\text {stab }} \cdot \nabla \vartheta_{h}\right)+\left(\nabla \delta \vartheta_{h}, \kappa_{h} \nabla \vartheta_{h}\right) \\
& \quad+\tau_{\vartheta}\left(c_{h} \mathbf{v}_{h}^{\text {stab }} \cdot \nabla \delta \vartheta_{h}, \mathcal{P}_{h}^{\perp}\left(c_{h} \mathbf{v}_{h}^{\text {stab }} \cdot \nabla \vartheta_{h}\right)\right) \\
& \quad-\tau_{\vartheta}\left(c_{h} \mathbf{v}_{h}^{\text {stab }} \cdot \nabla \delta \vartheta_{h}, \mathcal{P}_{h}^{\perp}\left(r_{h}\right)\right) \\
& \quad-\tau_{\vartheta}\left(c_{h} \mathbf{v}_{h}^{\text {stab }} \cdot \nabla \delta \vartheta_{h}, \mathcal{P}_{h}^{\perp}\left(\mathcal{D}_{h}\right)\right)=F_{\vartheta}\left(\delta \vartheta_{h}\right) \quad \forall \delta \vartheta_{h}
\end{aligned}
$$

REMARK 6. Use of the finite element velocity, instead of the stabilized one, in the convective term of the temperature equation. Setting $\tau_{v}=0$ in the stabilized variational form of the energy balance equation, i.e. setting $\mathbf{v}_{h}^{\text {stab }}=\mathbf{v}_{h}$ in the convective term of the sub-grid scale temperature, the sub-grid scale stabilized velocity, pressure and temperature read 


$$
\begin{aligned}
\mathbf{v}_{h}^{\text {stab }} & :=\mathbf{v}_{h}+\tilde{\mathbf{v}}=\mathbf{v}_{h}+\tau_{v} \mathcal{P}_{h}^{\perp}\left(\nabla p_{h}+\mathbf{b}_{h}\right) \\
p_{h}^{\text {stab }} & :=p_{h} \\
\vartheta_{h}^{\text {stab }} & :=\vartheta_{h}+\tilde{\vartheta}=\vartheta_{h}-\tau_{\vartheta} \mathcal{P}_{h}^{\perp}\left(c_{h} \mathbf{v}_{h} \cdot \nabla \vartheta_{h}-r_{h}-\mathcal{D}_{h}\right)
\end{aligned}
$$

The quasi-static OSGS sub-grid scale stabilized mixed variational formulation reads

$$
\begin{aligned}
& \left(\nabla \cdot \delta \mathbf{v}_{h}, p_{h}\right)+\left(\nabla^{s} \delta \mathbf{v}_{h}, 2 \mu_{h} \nabla^{s} \mathbf{v}_{h}\right)=F_{v}\left(\delta \mathbf{v}_{h}\right) \quad \forall \delta \mathbf{v}_{h} \\
& \left(\delta p_{h}, \nabla \cdot \mathbf{v}_{h}\right) \\
& \quad-\tau_{v}\left(\nabla \delta p_{h}, \mathcal{P}_{h}^{\perp}\left(\nabla p_{h}\right)\right) \\
& \quad-\tau_{v}\left(\nabla \delta p_{h}, \mathcal{P}_{h}^{\perp}\left(\mathbf{b}_{h}\right)\right)=0 \quad \forall \delta p_{h} \\
& \left(\delta \vartheta_{h}, c_{h} \partial_{t} \vartheta_{h}\right)+\left(\delta \vartheta_{h}, c_{h} \mathbf{v}_{h} \cdot \nabla \vartheta_{h}\right)+\left(\nabla \delta \vartheta_{h}, \kappa_{h} \nabla \vartheta_{h}\right) \\
& \quad+\tau_{\vartheta}\left(c_{h} \mathbf{v}_{h} \cdot \nabla \delta \vartheta_{h}, \mathcal{P}_{h}^{\perp}\left(c_{h} \mathbf{v}_{h} \cdot \nabla \vartheta_{h}\right)\right) \\
& -\tau_{\vartheta}\left(c_{h} \mathbf{v}_{h} \cdot \nabla \delta \vartheta_{h}, \mathcal{P}_{h}^{\perp}\left(r_{h}\right)\right) \\
& -\tau_{\vartheta}\left(c_{h} \mathbf{v}_{h} \cdot \nabla \delta \vartheta_{h}, \mathcal{P}_{h}^{\perp}\left(\mathcal{D}_{h}\right)\right)=F_{\vartheta}\left(\delta \vartheta_{h}\right) \quad \forall \delta \vartheta_{h}
\end{aligned}
$$

REMARK 7. OSGS counterpart of the classical GLS/SUPG stabilization method. The OSGS counterpart of the GLS/SUPG stabilization method can be obtained neglecting the following terms in the stabilized variational equations given above:

$$
\begin{array}{r}
\tau_{v}\left(\nabla \delta p_{h}, \mathcal{P}_{h}^{\perp}\left(\mathbf{b}_{h}\right)\right)=0 \\
\tau_{\vartheta}\left(c_{h} \mathbf{v}_{h} \cdot \nabla \delta \vartheta_{h}, \mathcal{P}_{h}^{\perp}\left(r_{h}\right)\right)=0 \\
\tau_{\vartheta}\left(c_{h} \mathbf{v}_{h} \cdot \nabla \delta \vartheta_{h}, \mathcal{P}_{h}^{\perp}\left(\mathcal{D}_{h}\right)\right)=0
\end{array}
$$

The quasi-static OSGS sub-grid scale stabilized mixed variational formulation corresponds to the OSGS counterpart of the GLS/SUPG stabilized mixed variational formulation and reads

$$
\begin{aligned}
& \left(\nabla \cdot \delta \mathbf{v}_{h}, p_{h}\right)+\left(\nabla^{s} \delta \mathbf{v}_{h}, 2 \mu_{h} \nabla^{s} \mathbf{v}_{h}\right)=F_{v}\left(\delta \mathbf{v}_{h}\right) \quad \forall \delta \mathbf{v}_{h} \\
& \left(\delta p_{h}, \nabla \cdot \mathbf{v}_{h}\right) \\
& \quad-\tau_{v}\left(\nabla \delta p_{h}, \mathcal{P}_{h}^{\perp}\left(\nabla p_{h}\right)\right)=0 \quad \forall \delta p_{h} \\
& \left(\delta \vartheta_{h}, c_{h} \partial_{t} \vartheta_{h}\right)+\left(\delta \vartheta_{h}, c_{h} \mathbf{v}_{h} \cdot \nabla \vartheta_{h}\right)+\left(\nabla \delta \vartheta_{h}, \kappa_{h} \nabla \vartheta_{h}\right) \\
& \quad+\tau_{\vartheta}\left(c_{h} \mathbf{v}_{h} \cdot \nabla \delta \vartheta_{h}, \mathcal{P}_{h}^{\perp}\left(c_{h} \mathbf{v}_{h} \cdot \nabla \vartheta_{h}\right)\right)=F_{\vartheta}\left(\delta \vartheta_{h}\right) \quad \forall \delta \vartheta_{h}
\end{aligned}
$$

\subsubsection{Summary}

A summary of the main results obtained so far on the space-discrete setting is shown in Boxes 1-3. Box 1 collects the stabilized variational equations and stabilized unknowns for the ASGS and OSGS method using P1/P1/P1 linear velocity/linear pressure/linear temperature elements, 
assuming temperature dependent viscosity, thermal conductivity, and heat capacity. Box 2 collects the corresponding expressions for the particular simplified case of constant thermal conductivity, constant heat capacity and temperature independent viscosity. Finally, as a particular case of the expressions shown in Box 2, in Box 3 the stabilized velocity appearing in the convective terms of the temperature equation is substituted by its finite element component, and the projections of body forces, temperature transient term, heat source and dissipation are neglected. In this case, the classical GLS and SUPG stabilization methods are recovered as a particular case of the ASGS method.

Box 1. Quasi-static sub-grid scale stabilization. P1/P1/P1 linear velocity/linear pressure/linear temperature element. Temperature dependent viscosity, thermal conductivity and heat capacity.

\begin{tabular}{|c|}
\hline Sub-grid Scale Stabilized Mixed Variational Formulation \\
\hline $\begin{array}{l}\left(\nabla \cdot \delta \mathbf{v}_{h}, p_{h}^{\text {stab }}\right)+\left(\nabla^{s} \delta \mathbf{v}_{h}, 2 \mu_{h} \nabla^{s} \mathbf{v}_{h}\right) \\
\quad-\left(2 \nabla^{s} \delta \mathbf{v}_{h} \nabla \mu_{h}, \mathbf{v}_{h}^{\text {stab }}-\mathbf{v}_{h}\right)=F_{v}\left(\delta \mathbf{v}_{h}\right) \quad \forall \delta \mathbf{v}_{h} \\
\left(\delta p_{h}, \nabla \cdot \mathbf{v}_{h}\right)-\left(\nabla \delta p_{h}, \mathbf{v}_{h}^{\text {stab }}-\mathbf{v}_{h}\right)=0 \quad \forall \delta p_{h} \\
\left(\delta \vartheta_{h}, c_{h} \partial_{t} \vartheta_{h}\right)+\left(\delta \vartheta_{h}, c_{h} \mathbf{v}_{h}^{\text {stab }} \cdot \nabla \vartheta_{h}\right)+\left(\nabla \delta \vartheta_{h}, \kappa_{h} \nabla \vartheta_{h}\right) \\
\quad-\left(c_{h} \mathbf{v}_{h}^{\text {stab }} \cdot \nabla \delta \vartheta_{h}, \vartheta_{h}^{\text {stab }}-\vartheta_{h}\right)-\left(\nabla c_{h} \cdot \mathbf{v}_{h}^{\text {stab }} \delta \vartheta_{h}, \vartheta_{h}^{\text {stab }}-\vartheta_{h}\right) \\
-\left(\nabla \kappa_{h} \cdot \nabla \delta \vartheta_{h}, \vartheta_{h}^{\text {stab }}-\vartheta_{h}\right)=F_{\vartheta}\left(\delta \vartheta_{h}\right) \quad \forall \delta \vartheta_{h}\end{array}$ \\
\hline ASGS \\
\hline $\begin{aligned} \mathbf{v}_{h}^{\text {stab }} & :=\mathbf{v}_{h}+\tilde{\mathbf{v}}=\mathbf{v}_{h}+\tau_{v}\left(\nabla p_{h}+2 \nabla^{s} \mathbf{v}_{h} \nabla \mu_{h}+\mathbf{b}_{h}\right) \\
p_{h}^{\text {stab }} & :=p_{h} \\
\vartheta_{h}^{\text {stab }} & :=\vartheta_{h}+\tilde{\vartheta}=\vartheta_{h}-\tau_{\vartheta}\left(c_{h} \partial_{t} \vartheta_{h}+c_{h} \mathbf{v}_{h}^{\text {stab }} \cdot \nabla \vartheta_{h}-\nabla \kappa_{h} \cdot \nabla \vartheta_{h}-r_{h}-\mathcal{D}_{h}\right)\end{aligned}$ \\
\hline OSGS \\
\hline $\begin{aligned} \mathbf{v}_{h}^{\text {stab }} & :=\mathbf{v}_{h}+\tilde{\mathbf{v}}=\mathbf{v}_{h}+\tau_{v} \mathcal{P}_{h}^{\perp}\left(\nabla p_{h}+2 \nabla^{s} \mathbf{v}_{h} \nabla \mu_{h}+\mathbf{b}_{h}\right) \\
p_{h}^{\text {stab }} & :=p_{h} \\
\vartheta_{h}^{\text {stab }} & :=\vartheta_{h}+\tilde{\vartheta}=\vartheta_{h}-\tau_{\vartheta} \mathcal{P}_{h}^{\perp}\left(c_{h} \partial_{t} \vartheta_{h}+c_{h} \mathbf{v}_{h}^{\text {stab }} \cdot \nabla \vartheta_{h}-\nabla \kappa_{h} \cdot \nabla \vartheta_{h}-r_{h}-\mathcal{D}_{h}\right)\end{aligned}$ \\
\hline
\end{tabular}

Box 2. Quasi-static sub-grid scale stabilization. P1/P1/P1 linear velocity/linear pressure/linear temperature element. Constant heat capacity, constant thermal conductivity and temperature independent viscosity.

\begin{tabular}{|c|}
\hline Sub-grid Scale Stabilized Mixed Variational Formulation \\
\hline$\left(\nabla \cdot \delta \mathbf{v}_{h}, p_{h}^{\text {stab }}\right)+\left(\nabla^{s} \delta \mathbf{v}_{h}, 2 \mu_{h} \nabla^{s} \mathbf{v}_{h}\right)=F_{v}\left(\delta \mathbf{v}_{h}\right) \quad \forall \delta \mathbf{v}_{h}$ \\
$\left(\delta p_{h}, \nabla \cdot \mathbf{v}_{h}\right)-\left(\nabla \delta p_{h}, \mathbf{v}_{h}^{\text {stab }}-\mathbf{v}_{h}\right)=0 \quad \forall \delta p_{h}$ \\
$\left(\delta \vartheta_{h}, c_{h} \partial_{t} \vartheta_{h}\right)+\left(\delta \vartheta_{h}, c_{h} \mathbf{v}_{h}^{\text {stab }} \cdot \nabla \vartheta_{h}\right)+\left(\nabla \delta \vartheta_{h}, \kappa_{h} \nabla \vartheta_{h}\right)$ \\
$-\left(c_{h} \mathbf{v}_{h}^{\text {stab }} \cdot \nabla \delta \vartheta_{h}, \vartheta_{h}^{\text {stab }}-\vartheta_{h}\right)=F_{\vartheta}\left(\delta \vartheta_{h}\right) \quad \forall \delta \vartheta_{h}$ \\
\hline ASGS \\
\hline
\end{tabular}




\begin{tabular}{|l|}
\hline $\mathbf{v}_{h}^{\text {stab }}:=\mathbf{v}_{h}+\tau_{v}\left(\nabla p_{h}+\mathbf{b}_{h}\right)$ \\
$p_{h}^{\text {stab }}:=p_{h}$ \\
$\vartheta_{h}^{\text {stab }}:=\vartheta_{h}-\tau_{\vartheta}\left(c_{h} \partial_{t} \vartheta_{h}+c_{h} \mathbf{v}_{h}^{\text {stab }} \cdot \nabla \vartheta_{h}-r_{h}-\mathcal{D}_{h}\right)$ \\
\hline OSGS \\
\hline $\mathbf{v}_{h}^{\text {stab }}:=\mathbf{v}_{h}+\tau_{v} \mathcal{P}_{h}^{\perp}\left(\nabla p_{h}+\mathbf{b}_{h}\right)$ \\
$p_{h}^{\text {stab }}:=p_{h}$ \\
$\vartheta_{h}^{\text {stab }}:=\vartheta_{h}-\tau_{\vartheta} \mathcal{P}_{h}^{\perp}\left(c_{h} \mathbf{v}_{h}^{\text {stab }} \cdot \nabla \vartheta_{h}-r_{h}-\mathcal{D}_{h}\right)$ \\
\hline
\end{tabular}

Box 3. Quasi-static sub-grid scale stabilization. P1/P1/P1 linear velocity/linear pressure/linear temperature element. Constant heat capacity, constant thermal conductivity and temperature independent viscosity, finite element velocity in the convective terms, and projections of body forces, temperature transient term, heat source and dissipation are neglected.

\begin{tabular}{|c|}
\hline Sub-grid Scale Stabilized Mixed Variational Formulation \\
\hline$\left(\nabla \cdot \delta \mathbf{v}_{h}, p_{h}^{\text {stab }}\right)+\left(\nabla^{s} \delta \mathbf{v}_{h}, 2 \mu_{h} \nabla^{s} \mathbf{v}_{h}\right)=F_{v}\left(\delta \mathbf{v}_{h}\right) \quad \forall \delta \mathbf{v}_{h}$ \\
$\left(\delta p_{h}, \nabla \cdot \mathbf{v}_{h}\right)-\left(\nabla \delta p_{h}, \mathbf{v}_{h}^{\text {stab }}-\mathbf{v}_{h}\right)=0 \quad \forall \delta p_{h}$ \\
$\left(\delta \vartheta_{h}, c_{h} \partial_{t} \vartheta_{h}\right)+\left(\delta \vartheta_{h}, c_{h} \mathbf{v}_{h} \cdot \nabla \vartheta_{h}\right)+\left(\nabla \delta \vartheta_{h}, \kappa_{h} \nabla \vartheta_{h}\right)$ \\
$-\left(c_{h} \mathbf{v}_{h} \cdot \nabla \delta \vartheta_{h}, \vartheta_{h}^{\text {stab }}-\vartheta_{h}\right)=F_{\vartheta}\left(\delta \vartheta_{h}\right) \quad \forall \delta \vartheta_{h}$ \\
\hline GLS/SUPG \\
\hline $\mathbf{v}_{h}^{\text {stab }}:=\mathbf{v}_{h}+\tau_{v} \nabla p_{h}$ \\
$p_{h}^{\text {stab }}:=p_{h}$ \\
$\vartheta_{h}^{\text {stab }}:=\vartheta_{h}-\tau_{\vartheta} c_{h} \mathbf{v}_{h} \cdot \nabla \vartheta_{h}$ \\
\hline OSGS GLS/SUPG \\
\hline $\mathbf{v}_{h}^{\text {stab }}:=\mathbf{v}_{h}+\tau_{v} \mathcal{P}_{h}^{\perp}\left(\nabla p_{h}\right)$ \\
$p_{h}^{\text {stab }}:=p_{h}$ \\
$\vartheta_{h}^{\text {stab }}:=\vartheta_{h}-\tau_{\vartheta} \mathcal{P}_{h}^{\perp}\left(c_{h} \mathbf{v}_{h} \cdot \nabla \vartheta_{h}\right)$ \\
\hline
\end{tabular}

\section{Time discrete setting and product formula algorithm}

The sub-grid scale stabilized mixed variational formulation of the IBVP can be solved using a staggered algorithm arising from an isothermal operator split of the governing equations and a Product Formula Algorithm (PFA). Within this framework, a mechanical problem at constant temperature, with the velocity and pressure as mechanical variables, and a thermal problem at constant velocity and pressure, with the temperature as thermal variable, may be defined $[1,2,3,10,11,12]$.

Within a time discrete setting, a staggered algorithm is defined such that for an arbitrary time step, the isothermal mechanical problem is solved first, keeping constant the temperature from the previous time step. Once the mechanical variables have been updated, the thermal problem is solved next, keeping constant the updated mechanical variables $[1,2,3,10,11,12]$. 
$\mathrm{P} 1 / \mathrm{P} 1 / \mathrm{P} 1$ linear velocity/linear pressure/linear temperature elements are considered. A first-order Backward-Euler (BE) time integration scheme is used to integrate the local time variation of the temperature.

\subsection{Mechanical problem}

The fully discrete mechanical problem which arises from the application of a PFA to the isothermal operator split of the governing equations is defined by the discrete stabilized variational forms of the linear momentum balance and incompressibility equations given by

$$
\begin{aligned}
& \left(\nabla \cdot \delta \mathbf{v}_{h}, p_{h, n+1}^{\text {stab }}\right)+\left(\nabla^{s} \delta \mathbf{v}_{h}, 2 \mu_{h, n+1} \nabla^{s} \mathbf{v}_{h, n+1}\right) \\
& -\left(2 \nabla^{s} \delta \mathbf{v}_{h, n+1} \nabla \mu_{h, n+1}, \mathbf{v}_{h, n+1}^{\text {stab }}-\mathbf{v}_{h, n+1}\right)=F_{v, n+1}\left(\delta \mathbf{v}_{h}\right) \quad \forall \delta \mathbf{v}_{h} \\
& \left(\delta p_{h}, \nabla \cdot \mathbf{v}_{h, n+1}\right)-\left(\nabla \delta p_{h}, \mathbf{v}_{h, n+1}^{\text {stab }}-\mathbf{v}_{h, n+1}\right)=0 \quad \forall \delta p_{h}
\end{aligned}
$$

The fully discrete residual of the velocity equation at time $n+1$ is given by

$$
\mathbf{r}_{v, h, n+1}:=\nabla p_{h, n+1}+2 \nabla^{s} \mathbf{v}_{h, n+1} \nabla \mu_{h, n+1}+\mathbf{b}_{h, n+1}
$$

and the discrete velocity stabilization parameter at time $n+1$ is given by

$$
\tau_{v, n+1}=\left(c_{1} \frac{2 \mu_{h, n+1}}{h^{2}}\right)^{-1}=\frac{1}{c_{1}} \frac{h^{2}}{2 \mu_{h, n+1}}
$$

Note that for the mechanical problem, the temperature is kept constant and equal to the temperature at the end of the previous time step, denoted as $\vartheta_{h, n}$. Therefore, for the mechanical problem, the equivalent viscosity $\mu_{h, n+1}$ at time $n+1$ is evaluated as $\mu_{h, n+1}:=\mu\left(\mathbf{v}_{h, n+1}, \vartheta_{h, n}\right)$.

\subsubsection{Algebraic Sub-grid Scales (ASGS)}

The fully discrete sub-grid scale velocity at time $n+1$ for the ASGS is defined as

$$
\tilde{\mathbf{v}}_{n+1}=\tau_{v, n+1} \mathbf{r}_{v, h, n+1}=\tau_{v, n+1}\left(\nabla p_{h, n+1}+2 \nabla^{s} \mathbf{v}_{h, n+1} \nabla \mu_{h, n+1}+\mathbf{b}_{h, n+1}\right)
$$

The fully discrete sub-grid scale stabilized velocity and pressure at time $n+1$ for the ASGS are defined as

$$
\begin{aligned}
\mathbf{v}_{h, n+1}^{s t a b} & :=\mathbf{v}_{h, n+1}+\tau_{v, n+1} \mathbf{r}_{v, h, n+1} \\
& :=\mathbf{v}_{h, n+1}+\tau_{v, n+1}\left(\nabla p_{h, n+1}+2 \nabla^{s} \mathbf{v}_{h, n+1} \nabla \mu_{h, n+1}+\mathbf{b}_{h, n+1}\right) \\
p_{h, n+1}^{s t a b} & :=p_{h, n+1}
\end{aligned}
$$

Substituting the fully discrete stabilized velocity and pressure at time $n+1$ into the corresponding stabilized variational formulation of the linear momentum balance and incompressibility equations yields 


$$
\begin{aligned}
& \left(\nabla \cdot \delta \mathbf{v}_{h}, p_{h, n+1}\right)+\left(\nabla^{s} \delta \mathbf{v}_{h}, 2 \mu_{h, n+1} \nabla^{s} \mathbf{v}_{h, n+1}\right) \\
& \quad-\tau_{v, n+1}\left(2 \nabla^{s} \delta \mathbf{v}_{h} \nabla \mu_{h, n+1}, \nabla p_{h, n+1}\right) \\
& \quad-\tau_{v, n+1}\left(2 \nabla^{s} \delta \mathbf{v}_{h} \nabla \mu_{h, n+1}, 2 \nabla^{s} \mathbf{v}_{h, n+1} \nabla \mu_{h, n+1}\right) \\
& \quad-\tau_{v, n+1}\left(2 \nabla^{s} \delta \mathbf{v}_{h} \nabla \mu_{h, n+1}, \mathbf{b}_{h, n+1}\right)=F_{v, n+1}\left(\delta \mathbf{v}_{h}\right) \quad \forall \delta \mathbf{v}_{h} \\
& \left(\delta p_{h}, \nabla \cdot \mathbf{v}_{h, n+1}\right) \\
& \quad-\tau_{v, n+1}\left(\nabla \delta p_{h}, \nabla p_{h, n+1}\right) \\
& \quad-\tau_{v, n+1}\left(\nabla \delta p_{h}, 2 \nabla^{s} \mathbf{v}_{h, n+1} \nabla \mu_{h, n+1}\right) \\
& \quad-\tau_{v, n+1}\left(\nabla \delta p_{h}, \mathbf{b}_{h, n+1}\right)=0 \quad \forall \delta p_{h}
\end{aligned}
$$

For implementation purposes, a compact and convenient alternative expression can be obtained in terms of the residuals, yielding

$$
\begin{aligned}
& \left(\nabla \cdot \delta \mathbf{v}_{h}, p_{h, n+1}\right)+\left(\nabla^{s} \delta \mathbf{v}_{h}, 2 \mu_{h, n+1} \nabla^{s} \mathbf{v}_{h, n+1}\right) \\
& \quad-\tau_{v, n+1}\left(\nabla^{s} \delta \mathbf{v}_{h}, 2 \nabla \mu_{h, n+1} \otimes \mathbf{r}_{v, h, n+1}\right)=F_{v, n+1}\left(\delta \mathbf{v}_{h}\right) \quad \forall \delta \mathbf{v}_{h} \\
& \left(\delta p_{h}, \nabla \cdot \mathbf{v}_{h, n+1}\right) \\
& \quad-\tau_{v, n+1}\left(\nabla \delta p_{h}, \mathbf{r}_{v, h, n+1}\right)=0 \quad \forall \delta p_{h}
\end{aligned}
$$

\subsubsection{Orthogonal Sub-grid Scales (OSGS)}

The fully discrete sub-grid scale velocity at time $n+1$ for the OSGS is defined as

$$
\begin{aligned}
\tilde{\mathbf{v}}_{n+1} & =\tau_{v, n+1} \mathcal{P}_{h}^{\perp}\left(\mathbf{r}_{v, h, n+1}\right) \\
& =\tau_{v, n+1} \mathcal{P}_{h}^{\perp}\left(\nabla p_{h, n+1}+2 \nabla^{s} \mathbf{v}_{h, n+1} \nabla \mu_{h, n+1}+\mathbf{b}_{h, n+1}\right)
\end{aligned}
$$

The fully discrete sub-grid scale stabilized velocity and pressure at time $n+1$ for the OSGS are defined as

$$
\begin{aligned}
\mathbf{v}_{h, n+1}^{\text {stab }} & :=\mathbf{v}_{h, n+1}+\tau_{v, n+1} \mathcal{P}_{h}^{\perp}\left(\mathbf{r}_{v, h, n+1}\right) \\
& :=\mathbf{v}_{h, n+1}+\tau_{v, n+1} \mathcal{P}_{h}^{\perp}\left(\nabla p_{h, n+1}+2 \nabla^{s} \mathbf{v}_{h, n+1} \nabla \mu_{h, n+1}+\mathbf{b}_{h, n+1}\right) \\
p_{h, n+1}^{s t a b} & :=p_{h, n+1}
\end{aligned}
$$

Substituting the fully discrete stabilized velocity and pressure at time $n+1$ into the corresponding stabilized variational formulation of the linear momentum balance and incompressibility equations yields 


$$
\begin{aligned}
& \left(\nabla \cdot \delta \mathbf{v}_{h}, p_{h, n+1}\right)+\left(\nabla^{s} \delta \mathbf{v}_{h}, 2 \mu_{h, n+1} \nabla^{s} \mathbf{v}_{h, n+1}\right) \\
& \quad-\tau_{v, n+1}\left(2 \nabla^{s} \delta \mathbf{v}_{h} \nabla \mu_{h, n+1}, \mathcal{P}_{h}^{\perp}\left(\nabla p_{h, n+1}\right)\right) \\
& \quad-\tau_{v, n+1}\left(2 \nabla^{s} \delta \mathbf{v}_{h} \nabla \mu_{h, n+1}, \mathcal{P}_{h}^{\perp}\left(2 \nabla^{s} \mathbf{v}_{h, n+1} \nabla \mu_{h, n+1}\right)\right) \\
& \quad-\tau_{v, n+1}\left(2 \nabla^{s} \delta \mathbf{v}_{h} \nabla \mu_{h, n+1}, \mathcal{P}_{h}^{\perp}\left(\mathbf{b}_{h, n+1}\right)\right)=F_{v, n+1}\left(\delta \mathbf{v}_{h}\right) \quad \forall \delta \mathbf{v}_{h} \\
& \left(\delta p_{h}, \nabla \cdot \mathbf{v}_{h, n+1}\right) \\
& \quad-\tau_{v, n+1}\left(\nabla \delta p_{h}, \mathcal{P}_{h}^{\perp}\left(\nabla p_{h, n+1}\right)\right) \\
& \quad-\tau_{v, n+1}\left(\nabla \delta p_{h}, \mathcal{P}_{h}^{\perp}\left(2 \nabla^{s} \mathbf{v}_{h, n+1} \nabla \mu_{h, n+1}\right)\right) \\
& \quad-\tau_{v, n+1}\left(\nabla \delta p_{h}, \mathcal{P}_{h}^{\perp}\left(\mathbf{b}_{h, n+1}\right)\right)=0 \quad \forall \delta p_{h}
\end{aligned}
$$

For implementation purposes, a compact and convenient alternative expression can be obtained in terms of the residuals, yielding

$$
\begin{aligned}
& \left(\nabla \cdot \delta \mathbf{v}_{h}, p_{h, n+1}\right)+\left(\nabla^{s} \delta \mathbf{v}_{h}, 2 \mu_{h, n+1} \nabla^{s} \mathbf{v}_{h, n+1}\right) \\
& \quad-\tau_{v, n+1}\left(\nabla^{s} \delta \mathbf{v}_{h}, 2 \nabla \mu_{h, n+1} \otimes \mathcal{P}_{h}^{\perp}\left(\mathbf{r}_{v, h, n+1}\right)\right)=F_{v, n+1}\left(\delta \mathbf{v}_{h}\right) \quad \forall \delta \mathbf{v}_{h} \\
& \left(\delta p_{h}, \nabla \cdot \mathbf{v}_{h, n+1}\right) \\
& \quad-\tau_{v, n+1}\left(\nabla \delta p_{h}, \mathcal{P}_{h}^{\perp}\left(\mathbf{r}_{v, h, n+1}\right)\right)=0 \quad \forall \delta p_{h}
\end{aligned}
$$

Orthogonal projection of the residual of the velocity equation can be written as

$$
\mathcal{P}_{h}^{\perp}\left(\mathbf{r}_{v, h, n+1}\right):=\mathbf{r}_{v, h, n+1}-\mathcal{P}_{h}\left(\mathbf{r}_{v, h, n+1}\right):=\mathbf{r}_{v, h, n+1}-\boldsymbol{\pi}_{v, h, n+1}
$$

where we have introduced $\boldsymbol{\pi}_{v, h, n+1} \in \boldsymbol{L}^{2}(\Omega)$ as the $L^{2}$ projection of the discrete residual of the velocity equation at time $n+1$ onto the corresponding finite element space.

Adding the corresponding variational equation for the $L^{2}$ projection of the residual of the velocity equation onto the finite element space, the extended stabilized variational formulation of the mechanical problem can be written as

$$
\begin{aligned}
& \left(\nabla \cdot \delta \mathbf{v}_{h}, p_{h, n+1}\right)+\left(\nabla^{s} \delta \mathbf{v}_{h}, 2 \mu_{h, n+1} \nabla^{s} \mathbf{v}_{h, n+1}\right) \\
& \quad-\tau_{v, n+1}\left(\nabla^{s} \delta \mathbf{v}_{h}, 2 \nabla \mu_{h, n+1} \otimes\left(\mathbf{r}_{v, h, n+1}-\boldsymbol{\pi}_{v, h, n+1}\right)\right)=F_{v, n+1}\left(\delta \mathbf{v}_{h}\right) \quad \forall \delta \mathbf{v}_{h} \\
& \left(\delta p_{h}, \nabla \cdot \mathbf{v}_{h, n+1}\right) \\
& \quad-\tau_{v, n+1}\left(\nabla \delta p_{h}, \mathbf{r}_{v, h, n+1}-\boldsymbol{\pi}_{v, h, n+1}\right)=0 \quad \forall \delta p_{h} \\
& \left(\delta \pi_{v, h}, \mathbf{r}_{v, h, n+1}\right)-\left(\delta \boldsymbol{\pi}_{v, h}, \boldsymbol{\pi}_{v, h, n+1}\right)=0 \quad \forall \delta \boldsymbol{\pi}_{v, h}
\end{aligned}
$$

where $\delta \pi_{v, h} \in \boldsymbol{L}^{2}(\Omega)$ is the test function for the $L^{2}$ projection of the residual of the velocity equation onto the corresponding finite element space.

\subsection{Thermal problem}


The fully discrete thermal problem which arises from the application of a PFA to the isothermal operator split of the governing equations is defined by the stabilized variational form of the energy balance equation given by

$$
\begin{aligned}
& \frac{1}{\Delta t}\left(\delta \vartheta_{h}, c_{h, n+1}\left(\vartheta_{h, n+1}-\vartheta_{h, n}\right)\right)+\left(\delta \vartheta_{h}, c_{h, n+1} \mathbf{v}_{h, n+1}^{s t a b} \cdot \nabla \vartheta_{h, n+1}\right)+\left(\nabla \delta \vartheta_{h}, \kappa_{h, n+1} \nabla \vartheta_{h, n+1}\right) \\
& \quad-\left(c_{h, n+1} \mathbf{v}_{h, n+1}^{s t a b} \cdot \nabla \delta \vartheta_{h}, \vartheta_{h, n+1}^{s t a b}-\vartheta_{h, n+1}\right)-\left(\delta \vartheta_{h} \mathbf{v}_{h, n+1}^{s t a b} \cdot \nabla c_{h, n+1}, \vartheta_{h, n+1}^{s t a b}-\vartheta_{h, n+1}\right) \\
& \quad-\left(\nabla \kappa_{h, n+1} \cdot \nabla \delta \vartheta_{h,} \vartheta_{h, n+1}^{s t a b}-\vartheta_{h, n+1}\right)=F_{\vartheta, n+1}\left(\delta \vartheta_{h}\right) \quad \forall \delta \vartheta_{h}
\end{aligned}
$$

The fully discrete residual of the temperature equation at time $n+1$ is given by

$$
r_{\vartheta, h, n+1}:=\frac{1}{\Delta t} c_{h, n+1}\left(\vartheta_{h, n+1}-\vartheta_{h, n}\right)+c_{h, n+1} \mathbf{v}_{h, n+1}^{s t a} \cdot \nabla \vartheta_{h, n+1}-\nabla \kappa_{h, n+1} \cdot \nabla \vartheta_{h, n+1}-r_{h, n+1}-\mathcal{D}_{h, n+1}
$$

The discrete temperature stabilization parameter at time $n+1$ is given by

$$
\tau_{\vartheta, n+1}=\left(c_{1} \frac{\kappa_{h, n+1}}{h^{2}}+c_{2} \frac{c_{h, n+1}\left|\mathbf{v}_{h, n+1}^{\text {stab }}\right|}{h}\right)^{-1}
$$

Note that the convective terms and the time-discrete stabilization parameter for the temperature at time $n+1$ are computed with the discrete stabilized velocity at time $n+1$. Therefore the stabilized temperature at time $n+1$ is a function of the stabilized velocity at time $n+1$.

\subsubsection{Algebraic Sub-grid Scales (ASGS)}

The fully discrete sub-grid scale temperature at time $n+1$ for the ASGS is given by

$$
\tilde{\vartheta}_{n+1}=-\tau_{\vartheta, n+1} r_{\vartheta, h, n+1}
$$

The fully discrete sub-grid scale stabilized temperature at time $n+1$ for the ASGS is given by

$$
\vartheta_{h, n+1}^{s t a b}:=\vartheta_{h, n+1}-\tau_{\vartheta, n+1} r_{\vartheta, h, n+1}
$$

Substituting the fully discrete sub-grid scale stabilized temperature at time $n+1$ into the corresponding stabilized variational formulation of the energy balance equation yields 


$$
\begin{aligned}
& \frac{1}{\Delta t}\left(\delta \vartheta_{h}, c_{h, n+1}\left(\vartheta_{h, n+1}-\vartheta_{h, n}\right)\right)+\left(\delta \vartheta_{h}, c_{h, n+1} \mathbf{v}_{h, n+1}^{s t a b} \cdot \nabla \vartheta_{h, n+1}\right)+\left(\nabla \delta \vartheta_{h}, \kappa_{h, n+1} \nabla \vartheta_{h, n+1}\right) \\
& \quad+\tau_{\vartheta, n+1} \frac{1}{\Delta t}\left(c_{h, n+1} \mathbf{v}_{h, n+1}^{s t a b} \cdot \nabla \delta \vartheta_{h}, c_{h, n+1}\left(\vartheta_{h, n+1}-\vartheta_{h, n}\right)\right) \\
& \quad+\tau_{\vartheta, n+1}\left(c_{h, n+1} \mathbf{v}_{h, n+1}^{s t a b} \cdot \nabla \delta \vartheta_{h}, c_{h, n+1} \mathbf{v}_{h, n+1}^{s t a b} \cdot \nabla \vartheta_{h, n+1}\right) \\
& \quad-\tau_{\vartheta, n+1}\left(c_{h, n+1} \mathbf{v}_{h, n+1}^{s t a b} \cdot \nabla \delta \vartheta_{h}, \nabla \kappa_{h, n+1} \cdot \nabla \vartheta_{h, n+1}\right) \\
& \quad-\tau_{\vartheta, n+1}\left(c_{h, n+1} \mathbf{v}_{h, n+1}^{s t a b} \cdot \nabla \delta \vartheta_{h}, r_{h, n+1}+\mathcal{D}_{h, n+1}\right) \\
& \quad+\tau_{\vartheta, n+1} \frac{1}{\Delta t}\left(\delta \vartheta_{h}^{s t a b} \cdot \nabla c_{h, n+1}, c_{h, n+1}\left(\vartheta_{h, n+1}-\vartheta_{h, n}\right)\right) \\
& \quad+\tau_{\vartheta, n+1}\left(\delta \vartheta_{h}^{s t a b} \cdot \nabla c_{h, n+1}, c_{h, n+1} \mathbf{v}_{h, n+1}^{s t a b} \cdot \nabla \vartheta_{h, n+1}\right) \\
& \quad-\tau_{\vartheta, n+1}\left(\delta \vartheta_{h}^{s t a b} \cdot \nabla c_{h, n+1}, \nabla \kappa_{h, n+1} \cdot \nabla \vartheta_{h, n+1}\right) \\
& \quad-\tau_{\vartheta, n+1}\left(\delta \vartheta_{h}^{s t a b} \cdot \nabla c_{h, n+1}, r_{h, n+1}+\mathcal{D}_{h, n+1}\right) \\
& \quad+\tau_{\vartheta, n+1} \frac{1}{\Delta t}\left(\nabla \kappa_{h, n+1} \cdot \nabla \delta \vartheta_{h}, c_{h, n+1}\left(\vartheta_{h, n+1}-\vartheta_{h, n}\right)\right) \\
& \quad+\tau_{\vartheta, n+1}\left(\nabla \kappa_{h, n+1} \cdot \nabla \delta \vartheta_{h}, c_{h, n+1} \mathbf{v}_{h, n+1}^{s t a b} \cdot \nabla \vartheta_{h, n+1}\right) \\
& \quad-\tau_{\vartheta, n+1}\left(\nabla \kappa_{h, n+1} \cdot \nabla \delta \vartheta_{h}, \nabla \kappa_{h, n+1} \cdot \nabla \vartheta_{h, n+1}\right) \\
& \quad-\tau_{\vartheta, n+1}\left(\nabla \kappa_{h, n+1} \cdot \nabla \delta \vartheta_{h}, r_{h, n+1}+\mathcal{D}_{h, n+1}\right)=F_{\vartheta, n+1}\left(\delta \vartheta_{h}\right) \quad \forall \delta \vartheta_{h}
\end{aligned}
$$

For implementation purposes, a compact and convenient alternative expression can be obtained in terms of the residual of the temperature equation, yielding

$$
\begin{aligned}
& \frac{1}{\Delta t}\left(\delta \vartheta_{h}, c_{h, n+1}\left(\vartheta_{h, n+1}-\vartheta_{h, n}\right)\right)+\left(\delta \vartheta_{h}, c_{h, n+1} \mathbf{v}_{h, n+1}^{s t a b} \cdot \nabla \vartheta_{h, n+1}\right)+\left(\nabla \delta \vartheta_{h}, \kappa_{h, n+1} \nabla \vartheta_{h, n+1}\right) \\
& \quad+\tau_{\vartheta, n+1}\left(\nabla \delta \vartheta_{h}, c_{h, n+1} \mathbf{v}_{h, n+1}^{s t a b} r_{\vartheta, h, n+1}\right) \\
& \quad+\tau_{\vartheta, n+1}\left(\delta \vartheta_{h}, \mathbf{v}_{h, n+1}^{s t a b} \cdot \nabla c_{h, n+1} r_{\vartheta, h, n+1}\right) \\
& \quad+\tau_{\vartheta, n+1}\left(\nabla \delta \vartheta_{h}, \nabla \kappa_{h, n+1} r_{\vartheta, h, n+1}\right)=F_{\vartheta, n+1}\left(\delta \vartheta_{h}\right) \quad \forall \delta \vartheta_{h}
\end{aligned}
$$

Note that we need to store and update just the finite element component of the temperature at time $n$.

REMARK 8. Constant heat capacity and constant thermal conductivity. If the heat capacity and the thermal conductivity are constants, the following expressions hold

$$
\begin{aligned}
\nabla c_{h, n+1} & =\mathbf{0} \\
\nabla \kappa_{h, n+1} & =\mathbf{0}
\end{aligned}
$$

Using the expressions given above, the discrete sub-grid scale stabilized formulation takes the form 


$$
\begin{aligned}
& \frac{1}{\Delta t}\left(\delta \vartheta_{h}, c_{h, n+1}\left(\vartheta_{h, n+1}-\vartheta_{h, n}\right)\right)+\left(\delta \vartheta_{h}, c_{h, n+1} \mathbf{v}_{h, n+1}^{\text {stab }} \cdot \nabla \vartheta_{h, n+1}\right)+\left(\nabla \delta \vartheta_{h}, \kappa_{h, n+1} \nabla \vartheta_{h, n+1}\right) \\
& \quad+\tau_{\vartheta, n+1} \frac{1}{\Delta t}\left(c_{h, n+1} \mathbf{v}_{h, n+1}^{\text {stab }} \cdot \nabla \delta \vartheta_{h}, c_{h, n+1}\left(\vartheta_{h, n+1}-\vartheta_{h, n}\right)\right) \\
& \quad+\tau_{\vartheta, n+1}\left(c_{h, n+1} \mathbf{v}_{h, n+1}^{s t a b} \cdot \nabla \delta \vartheta_{h}, c_{h, n+1} \mathbf{v}_{h, n+1}^{s t a b} \cdot \nabla \vartheta_{h, n+1}\right) \\
& \quad-\tau_{\vartheta, n+1}\left(c_{h, n+1} \mathbf{v}_{h, n+1}^{\text {stab }} \cdot \nabla \delta \vartheta_{h}, r_{h, n+1}+\mathcal{D}_{h, n+1}\right)=F_{\vartheta, n+1}\left(\delta \vartheta_{h}\right) \quad \forall \delta \vartheta_{h}
\end{aligned}
$$

\subsubsection{Orthogonal Sub-grid Scales (OSGS)}

The fully discrete sub-grid scale temperature at time $n+l$ for the OSGS is given by

$$
\tilde{\vartheta}_{n+1}=-\tau_{\vartheta, n+1} \mathcal{P}_{h}^{\perp}\left(r_{\vartheta, h, n+1}\right)
$$

The fully discrete sub-grid scale stabilized temperature at time $n+1$ for the OSGS is given by

$$
\vartheta_{h, n+1}^{s t a b}:=\vartheta_{h, n+1}-\tau_{\vartheta, n+1} \mathcal{P}_{h}^{\perp}\left(r_{\vartheta, h, n+1}\right)
$$

Substituting the fully discrete stabilized temperature at time $n+1$ into the corresponding stabilized variational formulation of the energy balance equation yields

$$
\begin{aligned}
& \frac{1}{\Delta t}\left(\delta \vartheta_{h}, c_{h, n+1}\left(\vartheta_{h, n+1}-\vartheta_{h, n}\right)\right)+\left(\delta \vartheta_{h}, c_{h, n+1} \mathbf{v}_{h, n+1}^{s t a b} \cdot \nabla \vartheta_{h, n+1}\right)+\left(\nabla \delta \vartheta_{h}, \kappa_{h, n+1} \nabla \vartheta_{h, n+1}\right) \\
& \quad+\tau_{\vartheta, n+1} \frac{1}{\Delta t}\left(c_{h, n+1} \mathbf{v}_{h, n+1}^{s t a b} \cdot \nabla \delta \vartheta_{h}, \mathcal{P}_{h}^{\perp}\left(c_{h, n+1}\left(\vartheta_{h, n+1}-\vartheta_{h, n}\right)\right)\right) \\
& \quad+\tau_{\vartheta, n+1}\left(c_{h, n+1} \mathbf{v}_{h, n+1}^{s t a b} \cdot \nabla \delta \vartheta_{h}, \mathcal{P}_{h}^{\perp}\left(c_{h, n+1} \mathbf{v}_{h, n+1}^{s t a b} \cdot \nabla \vartheta_{h, n+1}\right)\right) \\
& \quad-\tau_{\vartheta, n+1}\left(c_{h, n+1} \mathbf{v}_{h, n+1}^{s t a b} \cdot \nabla \delta \vartheta_{h}, \mathcal{P}_{h}^{\perp}\left(\nabla \kappa_{h, n+1} \cdot \nabla \vartheta_{h, n+1}\right)\right) \\
& \quad-\tau_{\vartheta, n+1}\left(c_{h, n+1} \mathbf{v}_{h, n+1}^{s t a b} \cdot \nabla \delta \vartheta_{h}, \mathcal{P}_{h}^{\perp}\left(r_{h, n+1}+\mathcal{D}_{h, n+1}\right)\right) \\
& \quad+\tau_{\vartheta, n+1} \frac{1}{\Delta t}\left(\delta \vartheta_{h} \mathbf{v}_{h, n+1}^{s t a b} \cdot \nabla c_{h, n+1}, \mathcal{P}_{h}^{\perp}\left(c_{h, n+1}\left(\vartheta_{h, n+1}-\vartheta_{h, n}\right)\right)\right) \\
& \quad+\tau_{\vartheta, n+1}\left(\delta \vartheta_{h}^{s t a b} \cdot \nabla c_{h, n+1}, \mathcal{P}_{h}^{\perp}\left(c_{h, n+1} \mathbf{v}_{h, n+1}^{s t a b} \cdot \nabla \vartheta_{h, n+1}\right)\right) \\
& \quad-\tau_{\vartheta, n+1}\left(\delta \vartheta_{h} \mathbf{v}_{h, n+1}^{s t a b} \cdot \nabla c_{h, n+1}^{s t a b}, \mathcal{P}_{h}^{\perp}\left(\nabla \kappa_{h, n+1} \cdot \nabla \vartheta_{h, n+1}\right)\right) \\
& +\tau_{\vartheta, n+1} \frac{1}{\Delta t}\left(\nabla \kappa_{h, n+1}, \nabla \delta \vartheta_{h}^{\perp}\left(r_{h, n+1}+\mathcal{D}_{h, n+1}\right)\right) \\
& \quad+\tau_{\vartheta, n+1}\left(\nabla \kappa_{h, n+1} \cdot \nabla \delta \vartheta_{h}, \mathcal{P}_{h}^{\perp}\left(c_{h, n+1}\left(\mathbf{v}_{h, n+1}^{s t a b} \cdot \nabla \vartheta_{h, n+1}\right)\right)\right. \\
& \quad-\tau_{\vartheta, n+1}\left(\nabla \kappa_{h, n+1} \cdot \nabla \delta \vartheta_{h}, \mathcal{P}_{h}^{\perp}\left(\nabla \kappa_{h, n+1} \cdot \nabla \vartheta_{h, n+1}\right)\right) \\
& \quad-\tau_{\vartheta, n+1}\left(\nabla \kappa_{h, n+1} \cdot \nabla \delta \vartheta_{h}, \mathcal{P}_{h}^{\perp}\left(r_{h, n+1}+\mathcal{D}_{h, n+1}\right)\right)=F_{\vartheta, n+1}\left(\delta \vartheta_{h}\right) \quad \forall \delta \vartheta_{h}
\end{aligned}
$$


For implementation purposes, a compact and convenient alternative expression can be obtained in terms of the residual of the temperature equation, yielding

$$
\begin{aligned}
& \frac{1}{\Delta t}\left(\delta \vartheta_{h}, c_{h, n+1}\left(\vartheta_{h, n+1}-\vartheta_{h, n}\right)\right)+\left(\delta \vartheta_{h}, c_{h, n+1} \mathbf{v}_{h, n+1}^{s t a b} \cdot \nabla \vartheta_{h, n+1}\right)+\left(\nabla \delta \vartheta_{h}, \kappa_{h, n+1} \nabla \vartheta_{h, n+1}\right) \\
& \quad+\tau_{\vartheta, n+1}\left(\nabla \delta \vartheta_{h}, c_{h, n+1} \mathbf{v}_{h, n+1}^{s t a b} \mathcal{P}_{h}^{\perp}\left(r_{\vartheta, h, n+1}\right)\right) \\
& \quad+\tau_{\vartheta, n+1}\left(\delta \vartheta_{h}, \mathbf{v}_{h, n+1}^{s t a b} \cdot \nabla c_{h, n+1} \mathcal{P}_{h}^{\perp}\left(r_{\vartheta, h, n+1}\right)\right) \\
& \quad+\tau_{\vartheta, n+1}\left(\nabla \delta \vartheta_{h}, \nabla \kappa_{h, n+1} \mathcal{P}_{h}^{\perp}\left(r_{\vartheta, h, n+1}\right)\right)=F_{\vartheta, n+1}\left(\delta \vartheta_{h}\right) \quad \forall \delta \vartheta_{h}
\end{aligned}
$$

The orthogonal projection of the residual of the temperature equation can be written as

$$
\mathcal{P}_{h}^{\perp}\left(r_{\vartheta, h, n+1}\right):=r_{\vartheta, h, n+1}-\mathcal{P}_{h}\left(r_{\vartheta, h, n+1}\right):=r_{\vartheta, h, n+1}-\pi_{\vartheta, h, n+1}
$$

where we have introduced $\pi_{\vartheta, h, n+1} \in L^{2}(\Omega)$ as the $L^{2}$ projection of the discrete residual of the temperature equation at time $n+1$ onto its corresponding finite element space.

Adding the corresponding variational equation for the $L^{2}$ projection of the residual of the temperature equation onto its corresponding finite element space, the extended stabilized variational formulation of the thermal problem can be written as

$$
\begin{aligned}
& \frac{1}{\Delta t}\left(\delta \vartheta_{h}, c_{h, n+1}\left(\vartheta_{h, n+1}-\vartheta_{h, n}\right)\right)+\left(\delta \vartheta_{h}, c_{h, n+1} \mathbf{v}_{h, n+1}^{s t a b} \cdot \nabla \vartheta_{h, n+1}\right)+\left(\nabla \delta \vartheta_{h}, \kappa_{h, n+1} \nabla \vartheta_{h, n+1}\right) \\
& \quad+\tau_{\vartheta, n+1}\left(\nabla \delta \vartheta_{h}, c_{h, n+1} \mathbf{v}_{h, n+1}^{s t a b}\left(r_{\vartheta, h, n+1}-\pi_{\vartheta, h, n+1}\right)\right) \\
& \quad+\tau_{\vartheta, n+1}\left(\delta \vartheta_{h}, \mathbf{v}_{h, n+1}^{s t a b} \cdot \nabla c_{h, n+1}\left(r_{\vartheta, h, n+1}-\pi_{\vartheta, h, n+1}\right)\right) \\
& \quad+\tau_{\vartheta, n+1}\left(\nabla \delta \vartheta_{h}, \nabla \kappa_{h, n+1}\left(r_{\vartheta, h, n+1}-\pi_{\vartheta, h, n+1}\right)\right)=F_{\vartheta, n+1}\left(\delta \vartheta_{h}\right) \quad \forall \delta \vartheta_{h} \\
& \left(\delta \pi_{\vartheta, h}, r_{\vartheta, h, n+1}\right)-\left(\delta \pi_{\vartheta, h}, \pi_{\vartheta, h, n+1}\right)=0 \quad \forall \delta \pi_{\vartheta, h}
\end{aligned}
$$

where $\delta \pi_{\vartheta, h} \in L^{2}(\Omega)$ is the test function for the $L^{2}$ projection of the residual of the temperature equation onto its corresponding finite element space.

REMARK 9. Constant heat capacity and constant thermal conductivity. If the heat capacity and the thermal conductivity are constant, the following expressions hold

$$
\begin{array}{r}
\mathcal{P}_{h}^{\perp}\left(c_{h, n+1}\left(\vartheta_{h, n+1}-\vartheta_{h, n}\right)\right)=0 \\
\nabla c_{h, n+1}=\mathbf{0} \\
\nabla \kappa_{h, n+1}=\mathbf{0}
\end{array}
$$

Using the expressions given above, the discrete sub-grid scale stabilized formulation takes the form 


$$
\begin{aligned}
& \frac{1}{\Delta t}\left(\delta \vartheta_{h}, c_{h, n+1}\left(\vartheta_{h, n+1}-\vartheta_{h, n}\right)\right)+\left(\delta \vartheta_{h}, c_{h, n+1} \mathbf{v}_{h, n+1}^{s t a b} \cdot \nabla \vartheta_{h, n+1}\right)+\left(\nabla \delta \vartheta_{h}, \kappa_{h, n+1} \nabla \vartheta_{h, n+1}\right) \\
& \quad+\tau_{\vartheta, n+1}\left(c_{h, n+1} \mathbf{v}_{h, n+1}^{s t a b} \cdot \nabla \delta \vartheta_{h}, \mathcal{P}_{h}^{\perp}\left(c_{h, n+1} \mathbf{v}_{h, n+1}^{s t a b} \cdot \nabla \vartheta_{h, n+1}\right)\right) \\
& \quad-\tau_{\vartheta, n+1}\left(c_{h, n+1} \mathbf{v}_{h, n+1}^{s t a b} \cdot \nabla \delta \vartheta_{h}, \mathcal{P}_{h}^{\perp}\left(r_{h, n+1}+\mathcal{D}_{h, n+1}\right)\right)=F_{\vartheta, n+1}\left(\delta \vartheta_{h}\right) \quad \forall \delta \vartheta_{h}
\end{aligned}
$$

\subsection{Summary}

Boxes 4 and 5 collect the main expressions for the discrete ASGS and OSGS stabilized variational formulation for the mechanical and thermal problems, respectively, using P1/P1/P1 linear velocity/linear pressure/linear temperature elements.

\begin{tabular}{|c|}
\hline $\begin{array}{c}\text { Mechanical Problem } \\
\text { Discrete Sub-grid Scale Stabilized Mixed Variational Formulation }\end{array}$ \\
\hline $\begin{array}{l}\left(\nabla \cdot \delta \mathbf{v}_{h}, p_{h, n+1}^{\text {stab }}\right)+\left(\nabla^{s} \delta \mathbf{v}_{h}, 2 \mu_{h, n+1} \nabla^{s} \mathbf{v}_{h, n+1}\right) \\
\quad-\left(2 \nabla^{s} \delta \mathbf{v}_{h, n+1} \nabla \mu_{h, n+1}, \mathbf{v}_{h, n+1}^{\text {stab }}-\mathbf{v}_{h, n+1}\right)=F_{v, n+1}\left(\delta \mathbf{v}_{h}\right) \quad \forall \delta \mathbf{v}_{h} \\
\left(\delta p_{h}, \nabla \cdot \mathbf{v}_{h, n+1}\right)-\left(\nabla \delta p_{h}, \mathbf{v}_{h, n+1}^{s t a b}-\mathbf{v}_{h, n+1}\right)=0 \quad \forall \delta p_{h}\end{array}$ \\
\hline ASGS \\
\hline $\begin{aligned} \mathbf{v}_{h, n+1}^{s t a b} & :=\mathbf{v}_{h, n+1}+\tau_{v, n+1} \mathbf{r}_{v, h, n+1} \\
p_{h, n+1}^{\text {stab }} & :=p_{h, n+1}\end{aligned}$ \\
\hline $\begin{array}{l}\left(\nabla \cdot \delta \mathbf{v}_{h}, p_{h, n+1}\right)+\left(\nabla^{s} \delta \mathbf{v}_{h}, 2 \mu_{h, n+1} \nabla^{s} \mathbf{v}_{h, n+1}\right) \\
\quad-\tau_{v, n+1}\left(\nabla^{s} \delta \mathbf{v}_{h}, 2 \nabla \mu_{h, n+1} \otimes \mathbf{r}_{v, h, n+1}\right)=F_{v, n+1}\left(\delta \mathbf{v}_{h}\right) \quad \forall \delta \mathbf{v}_{h} \\
\left(\delta p_{h}, \nabla \cdot \mathbf{v}_{h, n+1}\right) \\
\quad-\tau_{v, n+1}\left(\nabla \delta p_{h}, \mathbf{r}_{v, h, n+1}\right)=0 \quad \forall \delta p_{h}\end{array}$ \\
\hline OSGS \\
\hline $\begin{array}{l}\mathbf{v}_{h, n+1}^{s t a b}:=\mathbf{v}_{h, n+1}+\tau_{v, n+1}\left(\mathbf{r}_{v, h, n+1}-\pi_{v, h, n+1}\right) \\
p_{h, n+1}^{s t a b}:=p_{h, n+1}\end{array}$ \\
\hline $\begin{array}{l}\left(\nabla \cdot \delta \mathbf{v}_{h}, p_{h, n+1}\right)+\left(\nabla^{s} \delta \mathbf{v}_{h}, 2 \mu_{h, n+1} \nabla^{s} \mathbf{v}_{h, n+1}\right) \\
\quad-\tau_{v, n+1}\left(\nabla^{s} \delta \mathbf{v}_{h}, 2 \nabla \mu_{h, n+1} \otimes\left(\mathbf{r}_{v, h, n+1}-\boldsymbol{\pi}_{v, h, n+1}\right)\right)=F_{v, n+1}\left(\delta \mathbf{v}_{h}\right) \quad \forall \delta \mathbf{v}_{h} \\
\left(\delta p_{h}, \nabla \cdot \mathbf{v}_{h, n+1}\right) \\
\quad-\tau_{v, n+1}\left(\nabla \delta p_{h}, \mathbf{r}_{v, h, n+1}-\boldsymbol{\pi}_{v, h, n+1}\right)=0 \quad \forall \delta p_{h} \\
\left(\delta \pi_{v, h}, \mathbf{r}_{v, h, n+1}\right)-\left(\delta \pi_{v, h}, \boldsymbol{\pi}_{v, h, n+1}\right)=0 \quad \forall \delta \pi_{v, h}\end{array}$ \\
\hline
\end{tabular}

Box 4. Mechanical Problem: ASGS and OSGS stabilized variational formulation for P1/P1/P1 linear velocity/linear pressure/linear temperature elements.

Box 5. Thermal Problem: ASGS and OSGS stabilized variational formulation for P1/P1/P1 linear velocity/linear pressure/linear temperature elements. 


\begin{tabular}{|c|}
\hline $\begin{array}{l}\frac{1}{\Delta t}\left(\delta \vartheta_{h}, c_{h, n+1}\left(\vartheta_{h, n+1}-\vartheta_{h, n}\right)\right)+\left(\delta \vartheta_{h}, c_{h, n+1} \mathbf{v}_{h, n+1}^{s t a b} \cdot \nabla \vartheta_{h, n+1}\right)+\left(\nabla \delta \vartheta_{h}, \kappa_{h, n+1} \nabla \vartheta_{h, n+1}\right) \\
\quad-\left(c_{h, n+1} \mathbf{v}_{h, n+1}^{s t a b} \cdot \nabla \delta \vartheta_{h}, \vartheta_{h, n+1}^{s t a b}-\vartheta_{h, n+1}\right)-\left(\delta \vartheta_{h} \mathbf{v}_{h, n+1}^{s t a b} \cdot \nabla c_{h, n+1}, \vartheta_{h, n+1}^{s t a b}-\vartheta_{h, n+1}\right) \\
\quad-\left(\nabla \kappa_{h, n+1} \cdot \nabla \delta \vartheta_{h,}, \vartheta_{h, n+1}^{s t a b}-\vartheta_{h, n+1}\right)=F_{\vartheta, n+1}\left(\delta \vartheta_{h}\right) \quad \forall \delta \vartheta_{h}\end{array}$ \\
\hline ASGS \\
\hline$\vartheta_{h, n+1}^{s t a b}:=\vartheta_{h, n+1}-\tau_{\vartheta, n+1} r_{\vartheta, h, n+1}$ \\
\hline $\begin{array}{l}\frac{1}{\Delta t}\left(\delta \vartheta_{h}, c_{h, n+1}\left(\vartheta_{h, n+1}-\vartheta_{h, n}\right)\right)+\left(\delta \vartheta_{h}, c_{h, n+1} \mathbf{v}_{h, n+1}^{s t a b} \cdot \nabla \vartheta_{h, n+1}\right)+\left(\nabla \delta \vartheta_{h}, \kappa_{h, n+1} \nabla \vartheta_{h, n+1}\right) \\
\quad+\tau_{\vartheta, n+1}\left(c_{h, n+1} \mathbf{v}_{h, n+1}^{s t a b} \cdot \nabla \delta \vartheta_{h}, r_{\vartheta, h, n+1}\right) \\
\quad+\tau_{\vartheta, n+1}\left(\delta \vartheta_{h} \mathbf{v}_{h, n+1}^{s t a b} \cdot \nabla c_{h, n+1}, r_{\vartheta, h, n+1}\right) \\
\quad+\tau_{\vartheta, n+1}\left(\nabla \kappa_{h, n+1} \cdot \nabla \delta \vartheta_{h,}, r_{\vartheta, h, n+1}\right)=F_{\vartheta, n+1}\left(\delta \vartheta_{h}\right) \quad \forall \delta \vartheta_{h}\end{array}$ \\
\hline OSGS \\
\hline$\vartheta_{h, n+1}^{s t a b}:=\vartheta_{h, n+1}-\tau_{\vartheta, n+1}\left(r_{\vartheta, h, n+1}-\pi_{\vartheta, h, n+1}\right)$ \\
\hline $\begin{array}{l}\frac{1}{\Delta t}\left(\delta \vartheta_{h}, c_{h, n+1}\left(\vartheta_{h, n+1}-\vartheta_{h, n}\right)\right)+\left(\delta \vartheta_{h}, c_{h, n+1} \mathbf{v}_{h, n+1}^{s t a b} \cdot \nabla \vartheta_{h, n+1}\right)+\left(\nabla \delta \vartheta_{h}, \kappa_{h, n+1} \nabla \vartheta_{h, n+1}\right) \\
\quad+\tau_{\vartheta, n+1}\left(c_{h, n+1} \mathbf{v}_{h, n+1}^{s t a b} \cdot \nabla \delta \vartheta_{h}, r_{\vartheta, h, n+1}-\pi_{\vartheta, h, n+1}\right) \\
\quad+\tau_{\vartheta, n+1}\left(\delta \vartheta_{h} \mathbf{v}_{h, n+1}^{\text {stab }} \cdot \nabla c_{h, n+1}, r_{\vartheta, h, n+1}-\pi_{\vartheta, h, n+1}\right) \\
\quad+\tau_{\vartheta, n+1}\left(\nabla \kappa_{h, n+1} \cdot \nabla \delta \vartheta_{h,}, r_{\vartheta, h, n+1}-\pi_{\vartheta, h, n+1}\right)=F_{\vartheta, n+1}\left(\delta \vartheta_{h}\right) \quad \forall \delta \vartheta_{h} \\
\left(\delta \pi_{\vartheta, h}, r_{\vartheta, h, n+1}\right)-\left(\delta \pi_{\vartheta, h}, \pi_{\vartheta, h, n+1}\right)=0 \quad \forall \delta \pi_{\vartheta, h}\end{array}$ \\
\hline
\end{tabular}

\section{Finite element implementation}

\subsection{Mechanical problem}

\subsubsection{Algebraic Sub-grid Scale (ASGS)}

Once the finite element discretization has been performed, the matrix form of the nonlinear algebraic system of equations emanating from the discrete ASGS sub-grid scale finite element stabilized variational form of the governing equations of the mechanical problem can be written as

$$
\begin{aligned}
& \mathrm{R}_{v, n+1}:=\mathrm{R}_{v}\left(\mathrm{~V}_{n+1}, \mathrm{P}_{n+1}\right)=0 \\
& \mathrm{R}_{p, n+1}:=\mathrm{R}_{p}\left(\mathrm{~V}_{n+1}, \mathrm{P}_{n+1}\right)=0
\end{aligned}
$$

where $\mathbf{R}_{v, n+1}$ and $\mathbf{R}_{p, n+1}$ are the global nodal residual vectors arising from the velocity and pressure equations at time $n+1$, respectively, and $\mathrm{V}_{n+1}$ and $\mathrm{P}_{n+1}$ are the vectors of nodal velocity and nodal pressure unknowns at time $n+1$, respectively.

The vectors of nodal mechanical unknowns $\mathrm{V}_{n+1}$ and $\mathrm{P}_{n+1}$ can be obtained through an incremental-iterative solution method. Using a Newton-Raphson algorithm, the nonlinear 
algebraic systems of equations has to be linearized at the iteration $i+1$ of the time step $n+1$, yielding

$$
\begin{aligned}
& \mathrm{R}_{v}\left(\mathrm{~V}_{n+1}^{(i)}, \mathrm{P}_{n+1}^{(i)}\right)+D \mathrm{R}_{v}\left(\mathrm{~V}_{n+1}^{(i)}, \mathrm{P}_{n+1}^{(i)}\right) \cdot \Delta \mathrm{V}_{n+1}^{(i)}+D \mathrm{R}_{v}\left(\mathrm{~V}_{n+1}^{(i)}, \mathrm{P}_{n+1}^{(i)}\right) \cdot \Delta \mathrm{P}_{n+1}^{(i)}=0 \\
& \mathrm{R}_{p}\left(\mathrm{~V}_{n+1}^{(i)}, \mathrm{P}_{n+1}^{(i)}\right)+D \mathrm{R}_{p}\left(\mathrm{~V}_{n+1}^{(i)}, \mathrm{P}_{n+1}^{(i)}\right) \cdot \Delta \mathrm{V}_{n+1}^{(i)}+D \mathrm{R}_{p}\left(\mathrm{~V}_{n+1}^{(i)}, \mathrm{P}_{n+1}^{(i)}\right) \cdot \Delta \mathrm{P}_{n+1}^{(i)}=0
\end{aligned}
$$

where the vectors of nodal increment of velocities $\Delta \mathrm{V}_{n+1}^{(i)}$ and nodal increment of pressures $\Delta \mathrm{P}_{n+1}^{(i)}$ are defined as

$$
\begin{aligned}
\Delta \mathrm{V}_{n+1}^{(i)} & =\mathrm{V}_{n+1}^{(i+1)}-\mathrm{V}_{n+1}^{(i)} \\
\Delta \mathrm{P}_{n+1}^{(i)} & =\mathrm{P}_{n+1}^{(i+1)}-\mathrm{P}_{n+1}^{(i)}
\end{aligned}
$$

Typical element entries for a node $\mathrm{A}$ of the global nodal residual vectors $\mathbf{R}_{v, n+1}$ and $\mathrm{R}_{p, n+1}$ are given by

$$
\begin{aligned}
\mathrm{R}_{v}^{A}\left(\mathrm{~V}_{n+1}^{(i)}, \mathrm{P}_{n+1}^{(i)}\right) & =\int_{\Omega_{e}} \nabla N_{v, A} p_{h, n+1}^{(i)} d \Omega \\
& +\int_{\Omega_{e}} \mathbf{B}_{A}^{T} 2 \mu_{h, n+1}^{(i)} \nabla^{s} \mathbf{v}_{h, n+1}^{(i)} d \Omega \\
& -\int_{\Omega_{e}} \tau_{v, n+1}^{(i)} \mathbf{B}_{A}^{T}\left(2 \nabla \mu_{h, n+1}^{(i)} \otimes \mathbf{r}_{v, h, n+1}^{(i)}\right) d \Omega \\
& -\int_{\Omega_{e}} N_{v, A} \mathbf{b}_{h, n+1} d \Omega \\
& -\int_{\partial_{\sigma} \Omega_{e}} N_{v, A} \overline{\mathbf{t}}_{h, n+1} d \partial_{\sigma} \Omega \\
\mathrm{R}_{p}^{A}\left(\mathrm{~V}_{n+1}^{(i)}, \mathrm{P}_{n+1}^{(i)}\right)= & \int_{\Omega_{e}} N_{p, A} r_{p, h, n+1}^{(i)} d \Omega \\
& -\int_{\Omega_{e}} \tau_{v, n+1}^{(i)} \nabla N_{p, A} \cdot \mathbf{r}_{v, h, n+1}^{(i)} d \Omega
\end{aligned}
$$

where $N_{v, A}$ and $N_{p, A}$ are the velocity and pressure interpolation shape functions for node A, respectively, and $\mathbf{B}_{A}$ is the velocity symmetric gradient interpolation matrix for node A.

\subsubsection{Orthogonal Sub-grid Scale (OSGS)}

Once the finite element discretization has been performed, the matrix form of the nonlinear algebraic system of equations emanating from the discrete OSGS sub-grid scale finite element stabilized variational form of the governing equations of the mechanical problem can be written as

$$
\begin{aligned}
& \mathrm{R}_{v, n+1}:=\mathrm{R}_{v}\left(\mathrm{~V}_{n+1}, \mathrm{P}_{n+1}, \sqsubset_{v, n+1}\right)=0 \\
& \mathrm{R}_{p, n+1}:=\mathrm{R}_{p}\left(\mathrm{~V}_{n+1}, \mathrm{P}_{n+1}, \sqsubset_{v, n+1}\right)=0 \\
& \mathrm{R}_{\pi_{v}, n+1}:=\mathrm{R}_{\pi_{v}}\left(\mathrm{~V}_{n+1}, \mathrm{P}_{n+1}, \sqsubset_{v, n+1}\right)=0
\end{aligned}
$$


where $\mathbf{R}_{v, n+1}, \mathbf{R}_{p, n+1}, \mathbf{R}_{\pi_{v}, n+1}$ are the global nodal residual vectors arising from the velocity, pressure, and velocity residual projection equations, respectively, at time $n+1$, and $\mathrm{V}_{n+1}, \mathrm{P}_{n+1}$, $\sqsubset_{v, n+1}$ are the vectors of nodal unknowns, velocity, pressure, and velocity residual projection, respectively, at time $n+1$.

The solution of the above system of equations can be obtained through a robust and efficient staggered algorithm by solving instead the slightly modified system of equations given by

$$
\begin{aligned}
\mathrm{R}_{v, n+1} & :=\mathrm{R}_{v}\left(\mathrm{~V}_{n+1}, \mathrm{P}_{n+1}, \sqsubset_{v, n}\right)=0 \\
\mathrm{R}_{p, n+1} & :=\mathrm{R}_{p}\left(\mathrm{~V}_{n+1}, \mathrm{P}_{n+1}, \sqsubset_{v, n}\right)=0 \\
\mathrm{R}_{\pi_{v}, n+1} & :=\mathrm{R}_{\pi_{v}}\left(\mathrm{~V}_{n+1}, \mathrm{P}_{n+1}, \sqsubset_{v, n+1}\right)=0
\end{aligned}
$$

The idea is to obtain first $\mathrm{V}_{n+1}$ and $\mathrm{P}_{n+1}$ by solving the first two systems of equations, keeping constants $ᄃ_{v, n}$ at the time step $n$. Once $\mathrm{V}_{n+1}$ and $\mathrm{P}_{n+1}$ have been obtained, they are substituted into the last two equations to obtain $\sqsubset_{v, n+1}$ at the time step $n+1$.

The unknowns $V_{n+1}$ and $P_{n+1}$ can be obtained using an incremental iterative NewtonRaphson algorithm, through an exact linearization of the first two systems of equations given by

$$
\begin{aligned}
& \mathrm{R}_{v}\left(\mathrm{~V}_{n+1}^{(i)}, \mathrm{P}_{n+1}^{(i)}, \sqsubset_{v, n}\right) \\
& +D \mathrm{R}_{v}\left(\mathrm{~V}_{n+1}^{(i)}, \mathrm{P}_{n+1}^{(i)}, \subset_{v}, n\right) \cdot \Delta_{n+1}^{(i)} \\
& +D \mathrm{R}_{v}\left(\mathrm{~V}_{n+1}^{(i)}, \mathrm{P}_{n+1}^{(i)}, \complement_{v}, n\right) \cdot \Delta_{n+1}^{(i)}=0 \\
& \mathrm{R}_{p}\left(\mathrm{~V}_{n+1}^{(i)}, \mathbf{P}_{n+1}^{(i)}, \sqsubset_{v, n}\right) \\
& +D \mathrm{R}_{p}\left(\mathrm{~V}_{n+1}^{(i)}, \mathrm{P}_{n+1}^{(i)}, \mathrm{C}_{v, n}\right) \cdot \Delta^{(i)}{ }_{n+1}^{(i)} \\
& +D \mathrm{R}_{p}\left(\mathrm{~V}_{n+1}^{(i)}, \mathrm{P}_{n+1}^{(i)}, \subset_{,},{ }^{,}\right) \cdot \Delta{ }_{n+1}^{(i)}=0
\end{aligned}
$$

where the vectors of nodal increment of velocities $\Delta \mathrm{V}_{n+1}^{(i)}$ and nodal increment of pressures $\Delta \mathrm{P}_{n+1}^{(i)}$ are defined as

$$
\begin{aligned}
& \Delta \mathrm{V}_{n+1}^{(i)}=\mathrm{V}_{n+1}^{(i+1)}-\mathrm{V}_{n+1}^{(i)} \\
& \Delta \mathrm{P}_{n+1}^{(i)}=\mathrm{P}_{n+1}^{(i+1)}-\mathrm{P}_{n+1}^{(i)}
\end{aligned}
$$

Typical element entries for a node $\mathrm{A}$ of the global nodal residual vectors $\mathbf{R}_{v, n+1}$ and $\mathbf{R}_{p, n+1}$ are given by 


$$
\begin{aligned}
\mathrm{R}_{v}^{A}\left(\mathrm{~V}_{n+1}^{(i)}, \mathrm{P}_{n+1}^{(i)}, \sqsubset_{v, n}\right)= & \int_{\Omega_{e}} \nabla N_{v, A} p_{h, n+1}^{(i)} d \Omega \\
& +\int_{\Omega_{e}} \mathbf{B}_{A}^{T} 2 \mu_{h, n+1}^{(i)} \nabla^{s} \mathbf{v}_{h, n+1}^{(i)} d \Omega \\
& -\int_{\Omega_{e}} \tau_{v, n+1}^{(i)} \mathbf{B}_{A}^{T}\left(2 \nabla \mu_{h, n+1}^{(i)} \otimes\left(\mathbf{r}_{v, h, n+1}^{(i)}-\boldsymbol{\pi}_{v, h, n}\right)\right) d \Omega \\
& -\int_{\Omega_{e}} N_{v, A} \mathbf{b}_{h, n+1} d \Omega \\
& -\int_{\partial_{\sigma} \Omega_{e}} N_{v, A} \overline{\mathbf{t}}_{h, n+1} d \partial_{\sigma} \Omega \\
\mathrm{R}_{p}^{A}\left(\mathrm{~V}_{n+1}^{(i)}, \mathrm{P}_{n+1}^{(i)}, \sqsubset_{v, n}\right)= & \int_{\Omega_{e}} N_{p, A} r_{p, h, n+1}^{(i)} d \Omega \\
& -\int_{\Omega_{e}} \tau_{v, n+1}^{(i)} \nabla N_{p, A} \cdot\left(\mathbf{r}_{v, h, n+1}^{(i)}-\boldsymbol{\pi}_{v, h, n}\right) d \Omega
\end{aligned}
$$

where $N_{v, A}$ and $N_{p, A}$ are the velocity and pressure interpolation shape functions for node A, respectively, and $\mathbf{B}_{A}$ is the velocity symmetric gradient interpolation matrix for node A.

Once $\mathrm{V}_{n+1}$ and $\mathrm{P}_{n+1}$ have been obtained they are substituted into the last two systems of equations to get $\sqsubset_{v, n+1}$ by solving the uncoupled algebraic linear system

$$
\mathrm{R}_{\pi_{v}, n+1}:=\mathrm{R}_{\pi_{v}}\left(\mathrm{~V}_{n+1}, \mathrm{P}_{n+1}, \check{\nu}_{v, n+1}\right)=0
$$

The computation of the projection $\sqsubset_{v, n+1}$ can be transformed in a straight-forward operation by considering appropriate lumped mass matrices, leading to an efficient solution algorithm, without loss of precision or robustness [4,5,22,23,29,30,35].

\subsection{Thermal problem}

\subsubsection{Algebraic Sub-grid Scale (ASGS)}

Once the finite element discretization has been performed, the matrix form of the nonlinear algebraic system of equations emanating from the discrete ASGS sub-grid scale finite element stabilized variational form of the governing equation of the thermal problem can be written as

$$
\mathrm{R}_{\vartheta, n+1}:=\mathrm{R}_{\vartheta}\left(\mathrm{T}_{n+1}\right)=0
$$

where $\mathbf{R}_{\vartheta, n+1}$ is the discrete global nodal residual vector arising from the temperature equation at time $n+1$, and $\mathrm{T}_{n+1}$ is the vector of nodal temperature unknowns at time $n+1$.

The vector of nodal temperature unknowns can be obtained through an incrementaliterative solution method. Using a Newton-Raphson algorithm, the nonlinear algebraic system of equations has to be linearized at the iteration $i+1$ of the time step $n+1$. The exact linearization of the above system of equations can be written as

$$
\mathrm{R}_{\vartheta}\left(\mathrm{T}_{n+1}^{(i)}\right)+D \mathrm{R}_{\vartheta}\left(\mathrm{T}_{n+1}^{(i)}\right) \cdot \Delta \mathrm{T}_{n+1}^{(i)}=0
$$

where the vector of nodal increment of temperature unknowns is defined as

$$
\Delta \mathbf{T}_{n+1}^{(i)}=\mathbf{T}_{n+1}^{(i+1)}-\mathrm{T}_{n+1}^{(i)}
$$


For the Quasi-static Sub-grid Scales (QSGS), a typical element entry for a node A of the global nodal residual vector $\mathbf{R}_{\vartheta, n+1}$ is given by

$$
\begin{aligned}
\mathrm{R}_{\vartheta}^{A}\left(\mathrm{~T}_{n+1}^{(i)}\right)= & \frac{1}{\Delta t} \int_{\Omega_{e}} N_{\vartheta, A} c_{h, n+1}^{(i)}\left(\vartheta_{h, n+1}^{(i)}-\vartheta_{h, n}\right) d \Omega \\
& +\int_{\Omega_{e}} N_{\vartheta, A} c_{h, n+1}^{(i)} \mathbf{v}_{h, n+1}^{s t a b} \cdot \nabla \vartheta_{h, n+1}^{(i)} d \Omega \\
& +\int_{\Omega_{e}} \nabla N_{\vartheta, A} \kappa_{h, n+1}^{(i)} \nabla \vartheta_{h, n+1}^{(i)} d \Omega \\
& +\int_{\Omega_{e}} \tau_{\vartheta, n+1}^{(i)} \nabla N_{\vartheta, A} \cdot c_{h, n+1}^{(i)} \mathbf{v}_{h, n+1}^{s t a b} r_{\vartheta, h, n+1}^{(i)} d \Omega \\
& +\int_{\Omega_{e}} \tau_{\vartheta, n+1}^{(i)} N_{\vartheta, A} \mathbf{v}_{h, n+1}^{s t a b} \cdot \nabla c_{h, n+1}^{(i)} r_{\vartheta, h, n+1}^{(i)} d \Omega \\
& +\int_{\Omega_{e}} \tau_{\vartheta, n+1}^{(i)} \nabla N_{\vartheta, A} \cdot \nabla \kappa_{h, n+1}^{(i)} r_{\vartheta, h, n+1}^{(i)} d \Omega \\
& -\int_{\Omega_{e}} N_{\vartheta, A} r_{h, n+1} d \Omega \\
& -\int_{\Omega_{e}} N_{\vartheta, A} \mathcal{D}_{h, n+1}^{(i)} d \Omega \\
& +\int_{\partial_{q} \Omega_{e}} N_{\vartheta, A} \bar{q}_{h, n+1} d \partial_{q} \Omega
\end{aligned}
$$

where $N_{\vartheta, A}$ is the temperature interpolation shape function for node A.

\subsubsection{Orthogonal Sub-grid Scale (OSGS)}

Once the finite element discretization has been performed, the matrix form of the nonlinear algebraic system of equations emanating from the discrete OSGS sub-grid scale finite element stabilized variational form of the governing equation of the thermal problem can be written as

$$
\begin{aligned}
\mathrm{R}_{\vartheta, n+1} & :=\mathrm{R}_{\vartheta}\left(\mathrm{T}_{n+1}, \digamma_{\vartheta, n+1}\right)=0 \\
\mathrm{R}_{\pi_{\vartheta}, n+1} & :=\mathrm{R}_{\pi_{\vartheta}}\left(\mathrm{T}_{n+1}, \digamma_{\vartheta, n+1}\right)=0
\end{aligned}
$$

where $\mathbf{R}_{\vartheta, n+1}$ and $\mathbf{R}_{\pi_{g}, n+1}$ are the global nodal residual vectors arising from the temperature and temperature residual projection equations at time $n+1$, respectively, and $\mathrm{T}_{n+1}$ and $\left\ulcorner_{\vartheta, n+1}\right.$ are the vectors of nodal unknowns, temperature and temperature modified residual projection at time $n+1$, respectively.

The solution of the above system of equations can be obtained through a robust and efficient staggered algorithm by solving the slightly modified systems of equations given by

$$
\begin{aligned}
\mathrm{R}_{\vartheta, n+1} & :=\mathrm{R}_{\vartheta}\left(\mathrm{T}_{n+1}, \check{\digamma}_{\vartheta, n}\right)=0 \\
\mathrm{R}_{\pi_{\vartheta}, n+1} & :=\mathrm{R}_{\pi_{\vartheta}}\left(\mathrm{T}_{n+1}, \check{\ulcorner}_{\vartheta, n+1}\right)=0
\end{aligned}
$$

The idea is to obtain first $T_{n+1}$ by solving the first system of equations, keeping constant $ᄃ_{\vartheta, n}$ at the time step $n$. Once $T_{n+1}$ has been obtained, it is substituted into the second equation to obtain $\sqsubset_{\vartheta, n+1}$ at the time step $n+1$. 
The vector of nodal temperature unknowns $T_{n+1}$ can be obtained using an incremental iterative Newton-Raphson algorithm, through an exact linearization of the first system of equations given by

$$
\mathrm{R}_{\vartheta}\left(\mathrm{T}_{n+1}^{(i)}, ᄃ_{\vartheta, n}\right)+D_{\vartheta}\left(\begin{array}{c}
(i) \\
n+1
\end{array}, \quad \vartheta, n\right) \cdot \Delta_{n+1}^{(i)}=0
$$

where the vector of nodal increment of temperatures $\Delta \mathrm{T}_{n+1}^{(i)}$ is defined as

$$
\Delta \mathrm{T}_{n+1}^{(i)}=\mathrm{T}_{n+1}^{(i+1)}-\mathrm{T}_{n+1}^{(i)}
$$

Once $\mathrm{T}_{n+1}$ has been obtained it is substituted into the second system of equations to get $ᄃ_{\vartheta, n+1}$ by solving the linear system

$$
\mathrm{R}_{\pi_{\vartheta}, n+1}:=\mathrm{R}_{\pi_{\vartheta}}\left(\mathrm{T}_{n+1}, ᄃ_{\vartheta, n+1}\right)=0
$$

Similarly as for the mechanical problem, the computation of the projection $ᄃ_{\vartheta, n+1}$ can be transformed in a straight-forward operation by considering an appropriate lumped mass matrix, leading to an efficient solution algorithm, without loss of precision or robustness $[4,5,22,23,29,30,35]$.

A typical element entry for a node $A$ of the global nodal residual vector $\mathbf{R}_{\vartheta, n+1}$ is given by

$$
\begin{aligned}
\mathrm{R}_{\vartheta}^{A}\left(\mathrm{~T}_{n+1}^{(i)},\left\llcorner_{\vartheta, n}\right)=\right. & \frac{1}{\Delta t} \int_{\Omega_{e}} N_{\vartheta, A} c_{h, n+1}^{(i)}\left(\vartheta_{h, n+1}^{(i)}-\vartheta_{h, n}\right) d \Omega \\
& +\int_{\Omega_{e}} N_{\vartheta, A} c_{h, n+1}^{(i)} \mathbf{v}_{h, n+1}^{s t a b} \cdot \nabla \vartheta_{h, n+1}^{(i)} d \Omega \\
& +\int_{\Omega_{e}} \nabla N_{\vartheta, A} \kappa_{h, n+1}^{(i)} \nabla \vartheta_{h, n+1}^{(i)} d \Omega \\
& +\int_{\Omega_{e}} \tau_{\vartheta, n+1}^{(i)} \nabla N_{\vartheta, A} \cdot c_{h, n+1}^{(i)} \mathbf{v}_{h, n+1}^{s t a b}\left(r_{\vartheta, h, n+1}^{(i)}-\pi_{\vartheta, h, n}\right) d \Omega \\
& +\int_{\Omega_{e}} \tau_{\vartheta, n+1}^{(i)} N_{\vartheta, A} \mathbf{v}_{h, n+1}^{s t a b} \cdot \nabla c_{h, n+1}^{(i)}\left(r_{\vartheta, h, n+1}^{(i)}-\pi_{\vartheta, h, n}\right) d \Omega \\
& +\int_{\Omega_{e}} \tau_{\vartheta, n+1}^{(i)} \nabla N_{\vartheta, A} \cdot \nabla \kappa_{h, n+1}^{(i)}\left(r_{\vartheta, h, n+1}^{(i)}-\pi_{\vartheta, h, n}\right) d \Omega \\
& -\int_{\Omega_{e}} N_{\vartheta, A} r_{h, n+1} d \Omega \\
& -\int_{\Omega_{e}} N_{\vartheta, A} \mathcal{D}_{h, n+1}^{(i)} d \Omega \\
& +\int_{\partial_{q} \Omega_{e}} N_{\vartheta, A} \bar{q}_{h, n+1} d \partial_{q} \Omega
\end{aligned}
$$

where $N_{\vartheta, A}$ is the temperature interpolation shape function for node A.

\section{Numerical examples}

In this section we present two numerical examples that illustrate the performance of the formulation presented above. The first example deals with the numerical simulation of a 
coupled thermomechanical flow in a 2D rectangular domain. Both steady-state and transient conditions are considered. The main goal of this numerical example is to compare the behavior of the different pressure and convection stabilization methods. First, using GLS method to stabilize the pressure, temperature distributions using SUPG and OSGS convection stabilization methods are compared. Secondly, using SUPG method to stabilize the convective term of the temperature equation, pressure and velocity contours using GLS and OSGS pressure stabilization methods are compared. The second numerical example shows the 3D numerical simulation of a FSW process. ASGS (GLS for the pressure equation and SUPG for the temperature equation) and OSGS (both for the pressure and the temperature equations) stabilization methods are used. The Newton-Raphson method, combined with a line search procedure, is used to solve the nonlinear equations emanating from the fully discrete stabilized variational equations. Calculations are performed with an enhanced version of the finite element code COMET [21] developed by the authors at the International Center for Numerical Methods in Engineering (CIMNE) in Barcelona. Pre- and post-processing is done with GiD [54], also developed at CIMNE.

\subsection{Coupled thermomechanical flow in a $2 D$ rectangular domain}

The first numerical example deals with the coupled thermo-mechanical analysis of the fluid flow and thermal convection along a $2 \mathrm{D}$ rectangular domain.

The geometry of the domain, length $4 \mathrm{~m}$ and height $1 \mathrm{~m}$, is shown on Figure 1 . Mechanical and thermal boundary conditions are schematically shown on Figure 2. Velocity is prescribed to zero along the lateral sides of the tube. The inflow velocity is prescribed in $\mathrm{x}-$ direction using a parabolic velocity given by $V=1.252 \mathrm{E}-02(y-0.5)^{2}+3.13 \mathrm{E}-03 \mathrm{~m} / \mathrm{s}$. The temperature is prescribed to $50{ }^{\circ} \mathrm{C}$ and $30^{\circ} \mathrm{C}$ at the inflow and outflow, respectively.

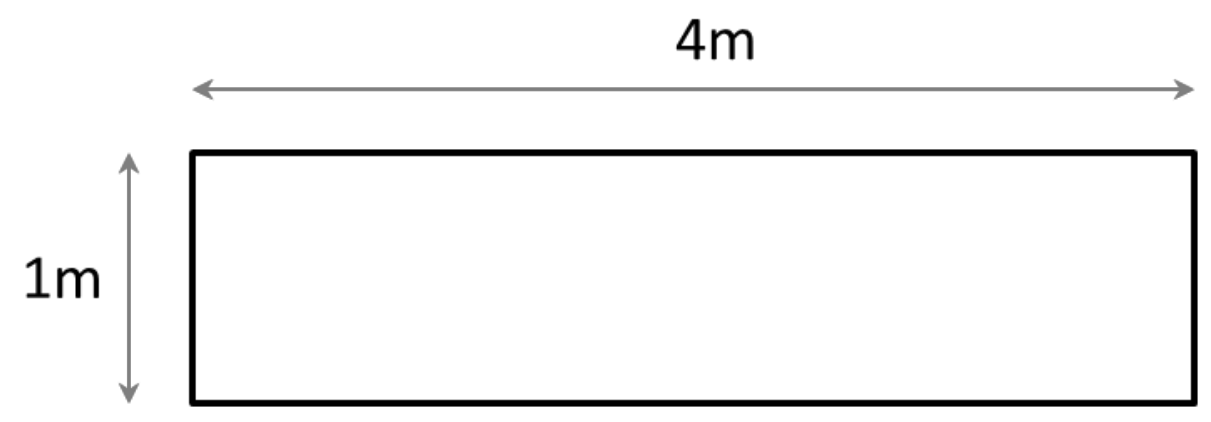

Figure 1. Geometry of the 2D rectangular domain 


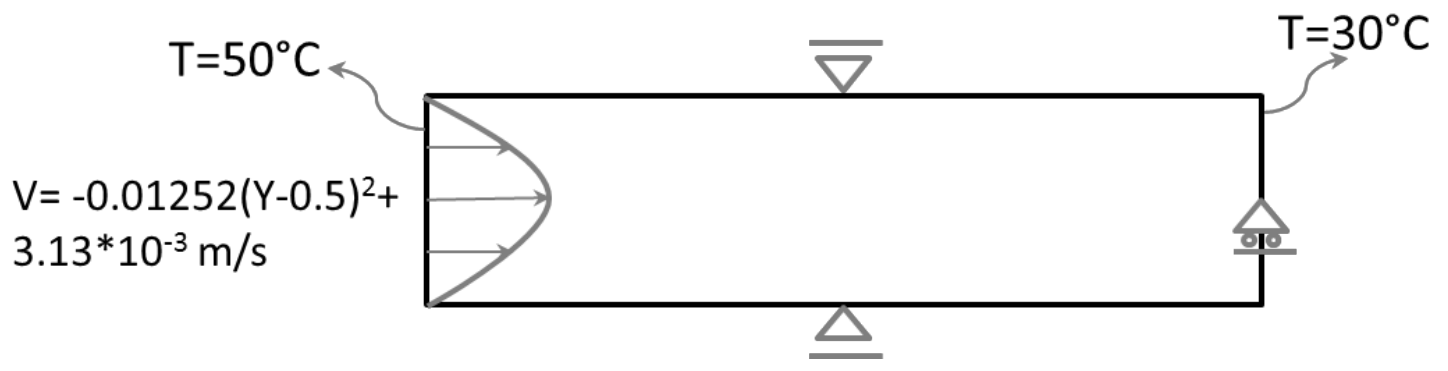

Figure 2. Prescribed velocity and temperature boundary conditions on the 2D rectangular domain

Table 1. Material thermal properties

\begin{tabular}{|c|c|c|}
\hline $\begin{array}{c}\text { Mass density } \\
{\left[\mathrm{Kg} \mathrm{m}^{-3}\right]}\end{array}$ & $\begin{array}{c}\text { Heat capacity } \\
{\left[\mathrm{J} \mathrm{Kg}^{-1}{ }^{\circ} \mathrm{C}^{-1}\right]}\end{array}$ & $\begin{array}{c}\text { Thermal conductivity } \\
{\left[\mathrm{W} \mathrm{m}^{-1}{ }^{\circ} \mathrm{C}^{-1}\right]}\end{array}$ \\
\hline $7.80 \mathrm{E}+03$ & $0.5 \mathrm{E}+03$ & 50 \\
\hline
\end{tabular}

Table 2. Norton-Hoff material model parameters

\begin{tabular}{|c|c|c|}
\hline Material Model & $\begin{array}{c}\text { Consistency parameter } \\
{[\mathrm{MPa} \mathrm{s}]}\end{array}$ & Rate sensitivity parameter \\
\hline Norton-Hoff & 100 & 1 \\
\hline
\end{tabular}

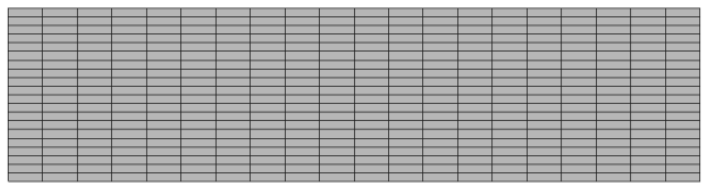

Figure 3. Finite element mesh consisting of $20 \times 20$ linear quadrilateral elements

A Norton-Hoff rigid visco-plastic constitutive model is considered. The thermal material properties and Norton-Hoff material model parameters are given in Tables 1 and 2, respectively.

The geometry is discretized with a finite element mesh consisting of $20 \times 20$ linear quadrilateral elements and 441 nodal points, as shown in Figure 3.

Two simulations, steady-state and dynamic for 70 time steps of 10 seconds are considered. First, the GLS stabilization method is used for the mechanical part and the thermal part is stabilized using SUPG and OSGS stabilization methods. The temperatures at time steps $10,40,70$ and the steady-state temperature along a line at the center of the tube are shown in Figure 4.

Temperatures provided by the SUPG and OSGS methods look very similar and only some slight differences can be seen, showing that OSGS provides slightly better results for the steady-state case. 


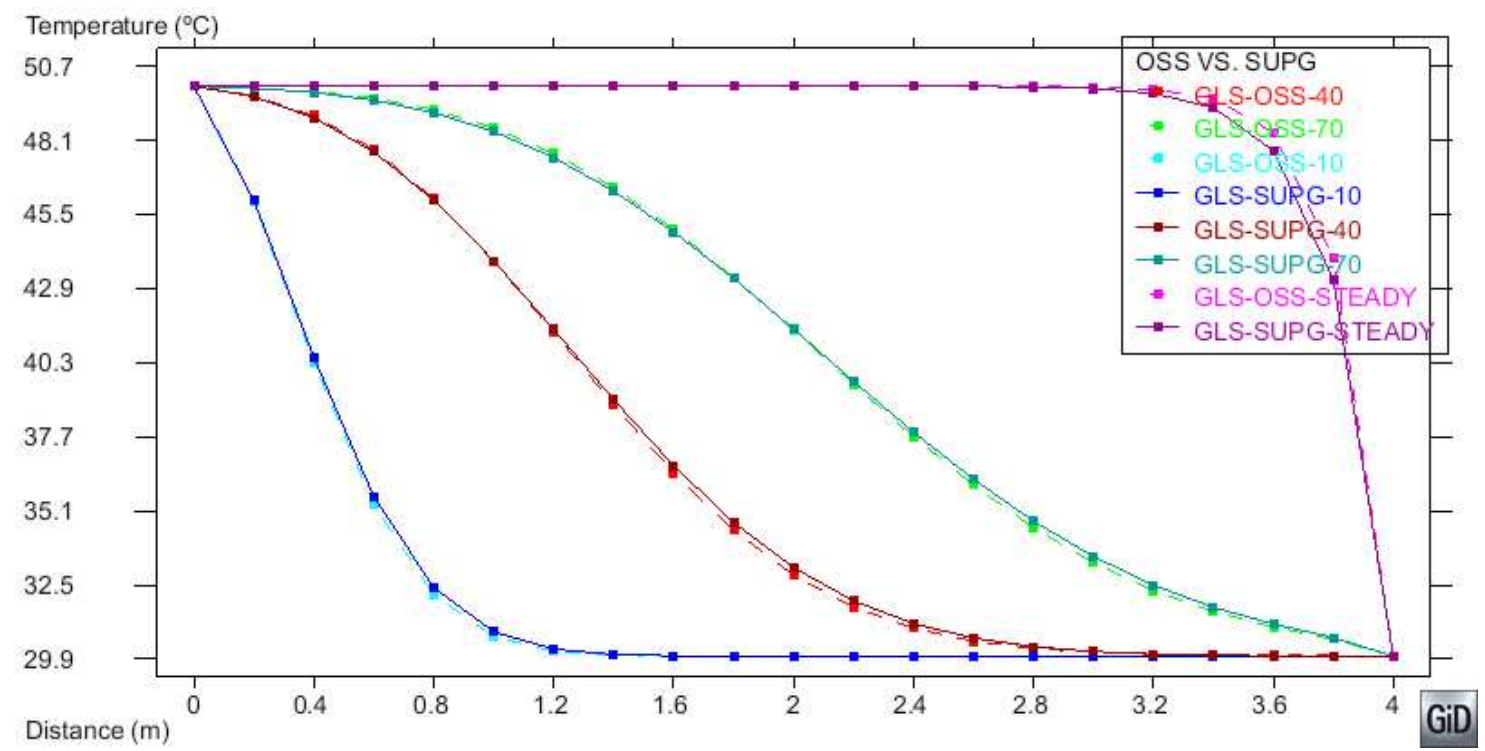

Figure 4. Comparison between the temperature distribution at the center line at different times $(10,40$ and 70$)$ and at the steady state, obtained using GLS pressure stabilization method and OSGS and SUPG convection stabilization methods

Next, the SUPG stabilization method is used for the thermal part and the mechanical part is stabilized once with the GLS and once with the OSGS stabilization methods. The pressure contour lines for both cases are shown in Figure 5 and 6.

A difference on the pressure contours obtained using GLS and OSGS stabilization methods is clearly shown at the inflow and outflow.

The velocity profiles at the inflow, outflow and center of the tube, using GLS and OSGS stabilization methods, are shown on Figures 7 and 8, respectively. The velocity contour lines for both cases are illustrated in Figure 9 and 10.

The figures clearly show that there is also an influence of the stabilization method on the velocity fields.
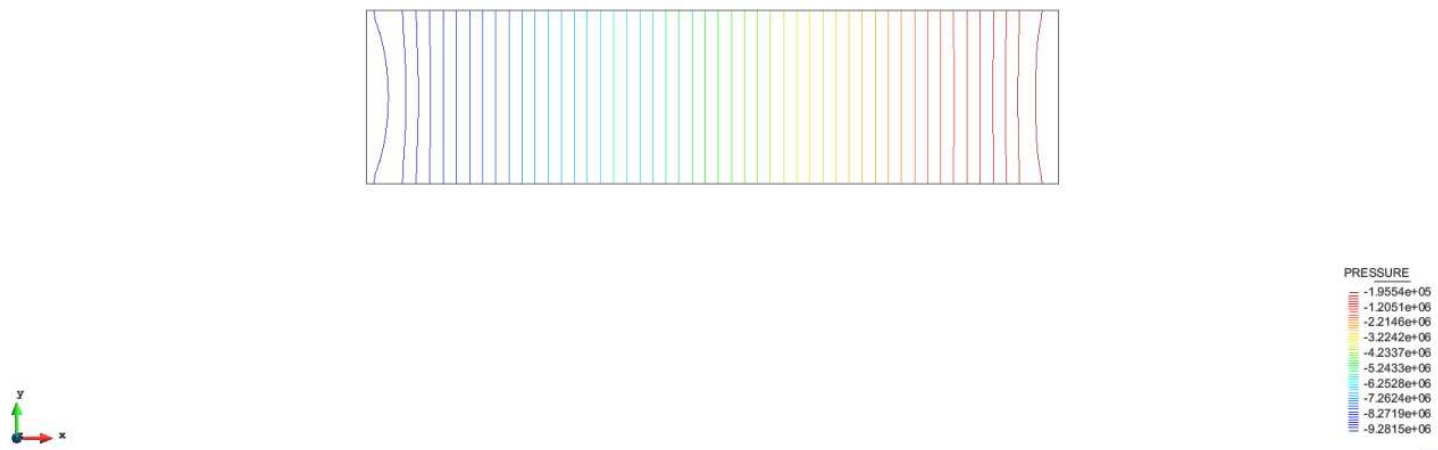

Figure 5. Pressure contour lines using SUPG convection stabilization method and GLS pressure stabilization method 


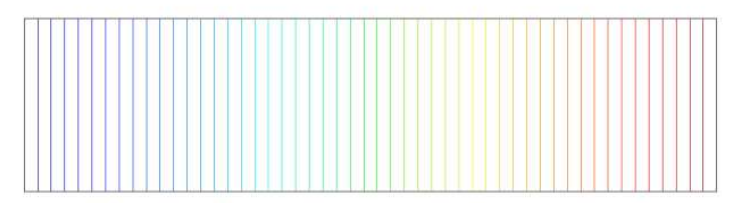

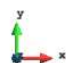

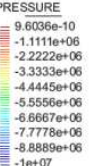

Figure 6. Pressure contour lines using SUPG convection stabilization method and OSGS pressure stabilization method

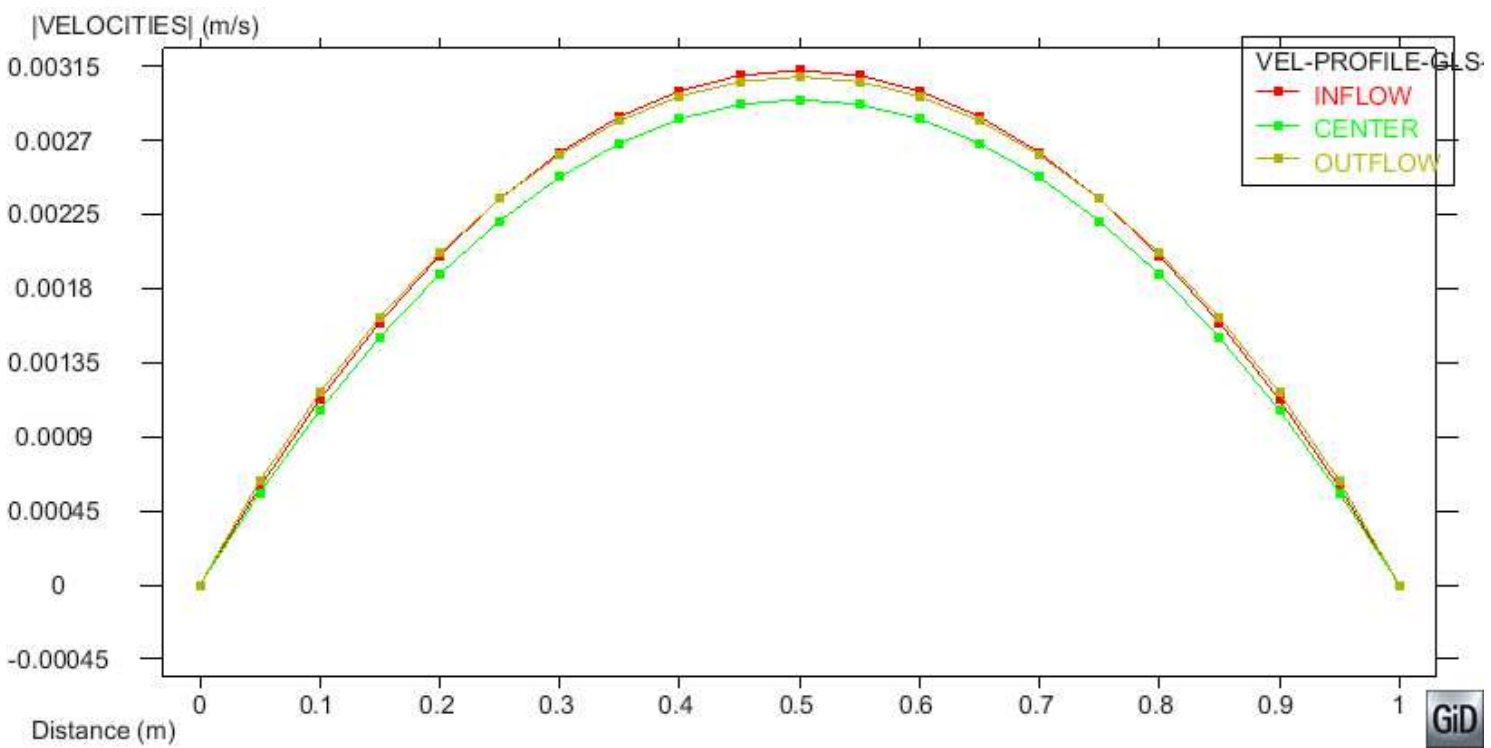

Figure 7. Velocity profiles at the inflow, outflow and center using SUPG convection stabilization method and GLS pressure stabilization method



Figure 8. Velocity profiles at the inflow, outflow and center using SUPG convection stabilization method and OSGS pressure stabilization method 




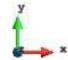

Figure 9. Velocity contour lines using SUPG convection stabilization method and GLS pressure stabilization method
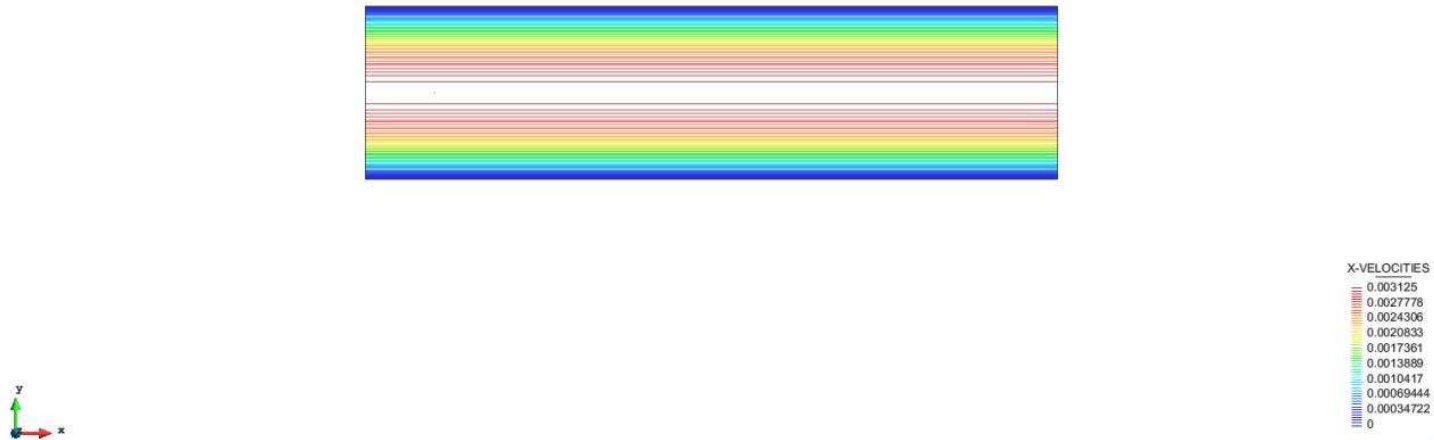

Figure 10. Velocity contour lines using SUPG convection stabilization method and OSGS pressure stabilization method

\subsection{D Transient coupled thermo-mechanical numerical simulation of a FSW process}

This example shows the 3D transient coupled thermo-mechanical numerical simulation of a FSW process. A finite element discretization of the work-pieces, cylindrical tool and back-plate has been considered in the simulation. A simplified geometrical model for the tool has been used, avoiding the finite element discretization of the shoulder scroll and the probe thread.

Geometrical data, process parameters, material properties and experimental results are taken from Zhu and Chao (2004) [83]. The diameters of the tool shoulder and tool probe are $19.05 \mathrm{~mm}$ (3/4 inches) and $6.35 \mathrm{~mm}$ (1/4 inches), respectively. The height of the tool shoulder and the depth of the tool probe are $50 \mathrm{~mm}$ and $3 \mathrm{~mm}$, respectively. The length of the workpieces, along the welding direction, is $300 \mathrm{~mm}$. The total width of the two work-pieces is 200 $\mathrm{mm}$ and the thickness is $3.18 \mathrm{~mm}$ (1/8 inches). The rotational velocity and advancing velocity of the tool are $500 \mathrm{rpm}$ and $101 \mathrm{~mm} / \mathrm{min}$ (4 inches/min), respectively. Material thermal properties are shown in Table 3.

The material of the work-pieces is an AISI $304 \mathrm{~L}$ and it has been modeled using a Sheppard-Wright material model. Material parameters for the Sheppard-Wright material model 
have been taken from Jorge Jr. and Balancin (2005) [61] and they are shown in Table 4. The tool and the back-plate have been modeled as thermo-rigid bodies.

Table 5 shows the boundary conditions used in the simulation for the mechanical and thermal problems.

Table 3. Material thermal properties for the work-pieces, tool and back-plate

\begin{tabular}{|c|c|c|c|c|c|}
\hline Body & $\begin{array}{c}\text { Mass } \\
\text { density } \\
{\left[\mathrm{Kg} \mathrm{m}^{-3}\right]}\end{array}$ & $\begin{array}{c}\text { Heat } \\
\text { capacity } \\
{\left[\mathrm{J} \mathrm{Kg}^{-1} \mathrm{C}^{-1}\right]}\end{array}$ & $\begin{array}{c}\text { Thermal } \\
\text { conductivity } \\
{\left[\mathrm{W} \mathrm{m}^{-1}{ }^{\circ} \mathrm{C}^{-1}\right]}\end{array}$ & $\begin{array}{c}\text { Convection heat } \\
\text { transfer coefficient } \\
{\left[\mathrm{W} \mathrm{m}^{-2}{ }^{\circ} \mathrm{C}^{-1}\right]}\end{array}$ & $\begin{array}{c}\text { Relative } \\
\text { emissivity }\end{array}$ \\
\hline Work-pieces & $8.00 \mathrm{E}+03$ & $0.51 \mathrm{E}+03$ & 21.4 & 10 & 0.17 \\
\hline Tool & $7.85 \mathrm{E}+03$ & $0.46 \mathrm{E}+03$ & 43.0 & 10 & 0.80 \\
\hline Back-plate & $7.85 \mathrm{E}+03$ & $0.46 \mathrm{E}+03$ & 43.0 & & \\
\hline
\end{tabular}

Table 4. Material parameters for the Sheppard-Wright material model for the work-pieces

\begin{tabular}{|c|c|c|c|c|c|}
\hline Material & Model & $\mathrm{A}$ & $\alpha\left[\mathrm{Pa}^{-1}\right]$ & $\mathrm{n}$ & $\mathrm{Q}\left[\mathrm{J} \mathrm{mol}^{-1}\right]$ \\
\hline AISI 304 L & Sheppard-Wright & $8.3 \mathrm{E}+15$ & $1.2 \mathrm{E}-08$ & 4.32 & $4.01 \mathrm{E}+05$ \\
\hline
\end{tabular}

Table 5. Boundary conditions for the mechanical and thermal problems

\begin{tabular}{|c|c|c|c|}
\hline Body & Boundary & Mechanical Problem & Thermal Problem \\
\hline \multirow{7}{*}{ Work-pieces } & Inlet & Prescribed minus advancing tool velocity & Prescribed temperature $=25^{\circ} \mathrm{C}$ \\
\hline & Outlet & Zero prescribed traction vector & Adiabatic \\
\hline & Sides & Prescribed minus advancing tool velocity & Adiabatic \\
\hline & Bottom & Prescribed minus advancing tool velocity & Thermal contact, HTC \\
\hline & Top & $\begin{array}{l}\text { Zero normal velocity and zero tangential } \\
\text { components of the traction vector }\end{array}$ & Convection+radiation \\
\hline & Shoulder & Frictional stick & Thermal contact, $\mathrm{HTC}=\infty$ \\
\hline & Probe & Frictional stick & Thermal contact, $\mathrm{HTC}=\infty$ \\
\hline \multirow{4}{*}{ Tool } & Side & \multirow{4}{*}{$\begin{array}{l}\text { Rigid with prescribed rotational tool } \\
\text { velocity at all the nodes of the tool }\end{array}$} & Convection+radiation \\
\hline & Top & & Convection+radiation \\
\hline & Shoulder & & Thermal contact, $\mathrm{HTC}=\infty$ \\
\hline & Probe & & Thermal contact, $\mathrm{HTC}=\infty$ \\
\hline \multirow{5}{*}{ Back-plate } & Inlet & \multirow{5}{*}{$\begin{array}{l}\text { Rigid with prescribed minus advancing } \\
\text { tool velocity at all the nodes of the back- } \\
\text { plate }\end{array}$} & Prescribed temperature $=25^{\circ} \mathrm{C}$ \\
\hline & Outlet & & Adiabatic \\
\hline & Sides & & Adiabatic \\
\hline & Bottom & & Adiabatic \\
\hline & Top & & Thermal contact, HTC \\
\hline
\end{tabular}

The prescribed tool advancing velocity has been imposed as a prescribed advancing velocity in the opposite direction to the work-pieces and back-plate. The prescribed tool rotational velocity has been applied to the tool.

Full stick friction conditions and thermal contact boundary conditions on the tool shoulder/work-pieces and tool probe/work-pieces contact interfaces have been considered, 
assuming an infinity value for the heat transfer coefficient. Thermal contact boundary conditions, with a contact heat transfer coefficient of $5.0 \mathrm{E}+3 \mathrm{~W} \mathrm{~m}^{-2}{ }^{\circ} \mathrm{C}^{-1}$, have been also considered on the contact surface between the work-pieces (bottom surface) and the back-plate (top surface). Convection/radiation boundary conditions have been considered on the top and lateral surfaces of the tool and on the top surface area of the work-pieces which is not in contact with the tool shoulder. Adiabatic boundary conditions have been considered on the outlet and the two external lateral surfaces of the work-pieces, as well as on the outlet, bottom and lateral surfaces of the back-plate. On the inlet surfaces of the work-pieces and the back-plate, the temperature has been prescribed to the environmental one. The initial and environmental temperature is $25^{\circ} \mathrm{C}$.

A finite element mesh consisting of 116,414 linear tetrahedra and 21,494 nodal points has been considered in the simulation. Prescribed advancing and rotational velocities have been imposed in an incremental way, using 1,000 time steps. A uniform time step of $0.1 \mathrm{sec}$ has been considered. The full FSW numerical simulation has been done using 2,000 time steps. Computing time for the whole simulation, using a personal computer with $2 \mathrm{~Gb}$ RAM, was around 74 hours.

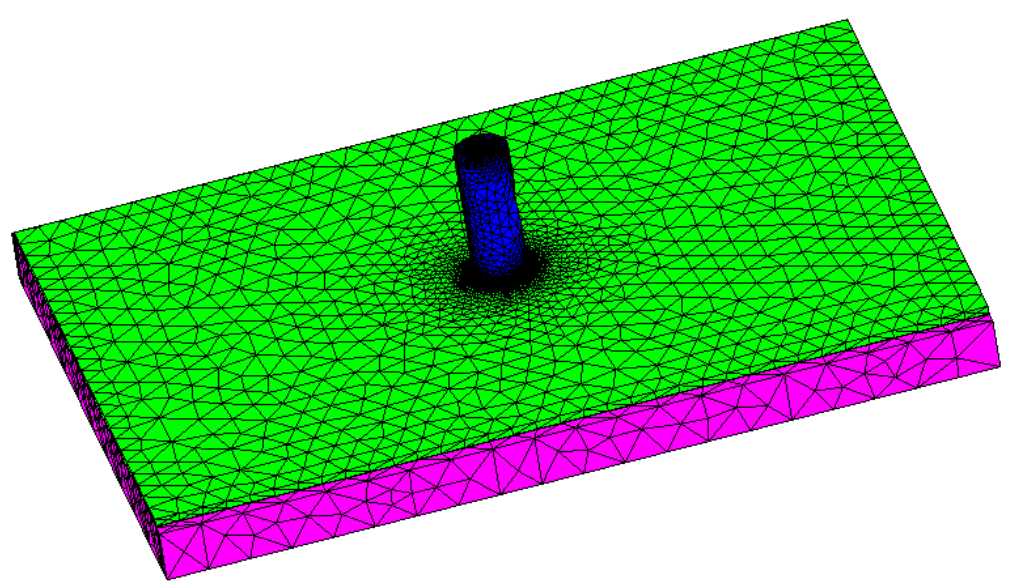

Figure 11. Finite element mesh of the tool, work-pieces and back-plate
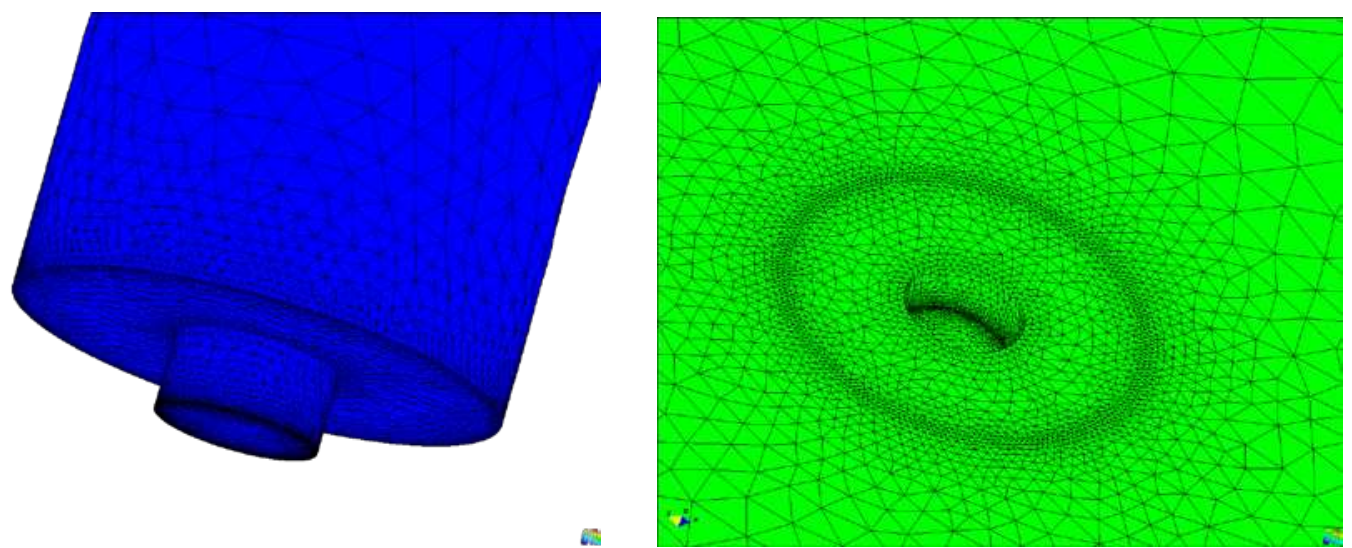

4.

03

Figure 12. Details of the finite element mesh of the tool (left) and work-pieces (right) 
Figure 11 shows a view of the finite element mesh of the tool, work-pieces and backplate. Figure 12 shows a detail of the finite element mesh used for the tool (left) and workpieces (right) in the stir area.

Figure 13 shows the temperature map distribution at the end of the numerical simulation. It can be clearly seen the convection effect due to the advancing tool speed. A detail of the temperature map on the tool and work-pieces at the welding line section at the end of the simulation is shown in Figure 14. Extremely high temperature gradients through the thickness of the work-pieces arise below the tool shoulder area.

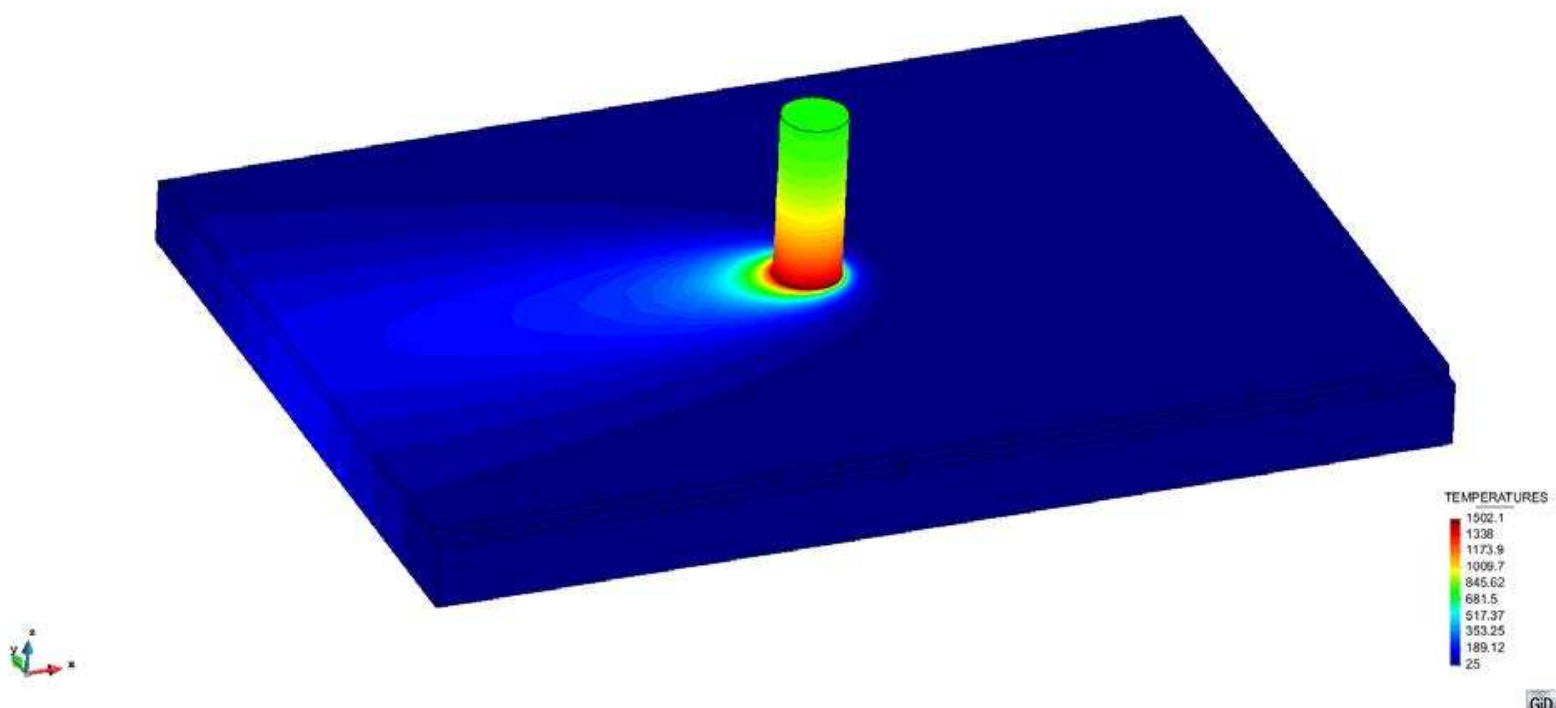

Figure 13. Temperature map distribution at the end of the simulation

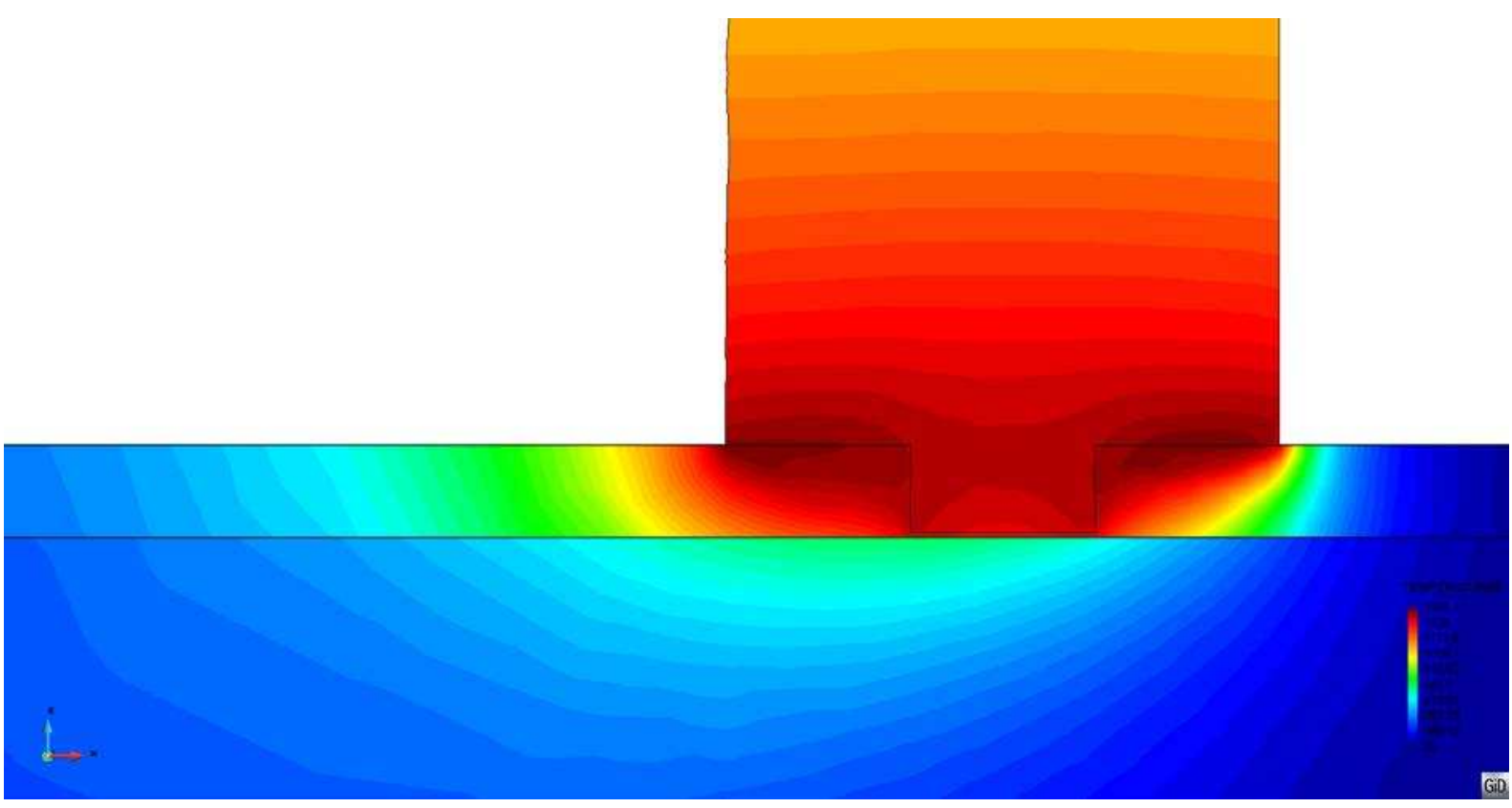

Figure 14. Zoom of the temperature map distribution at a cut of the welding line section at the end of the simulation 


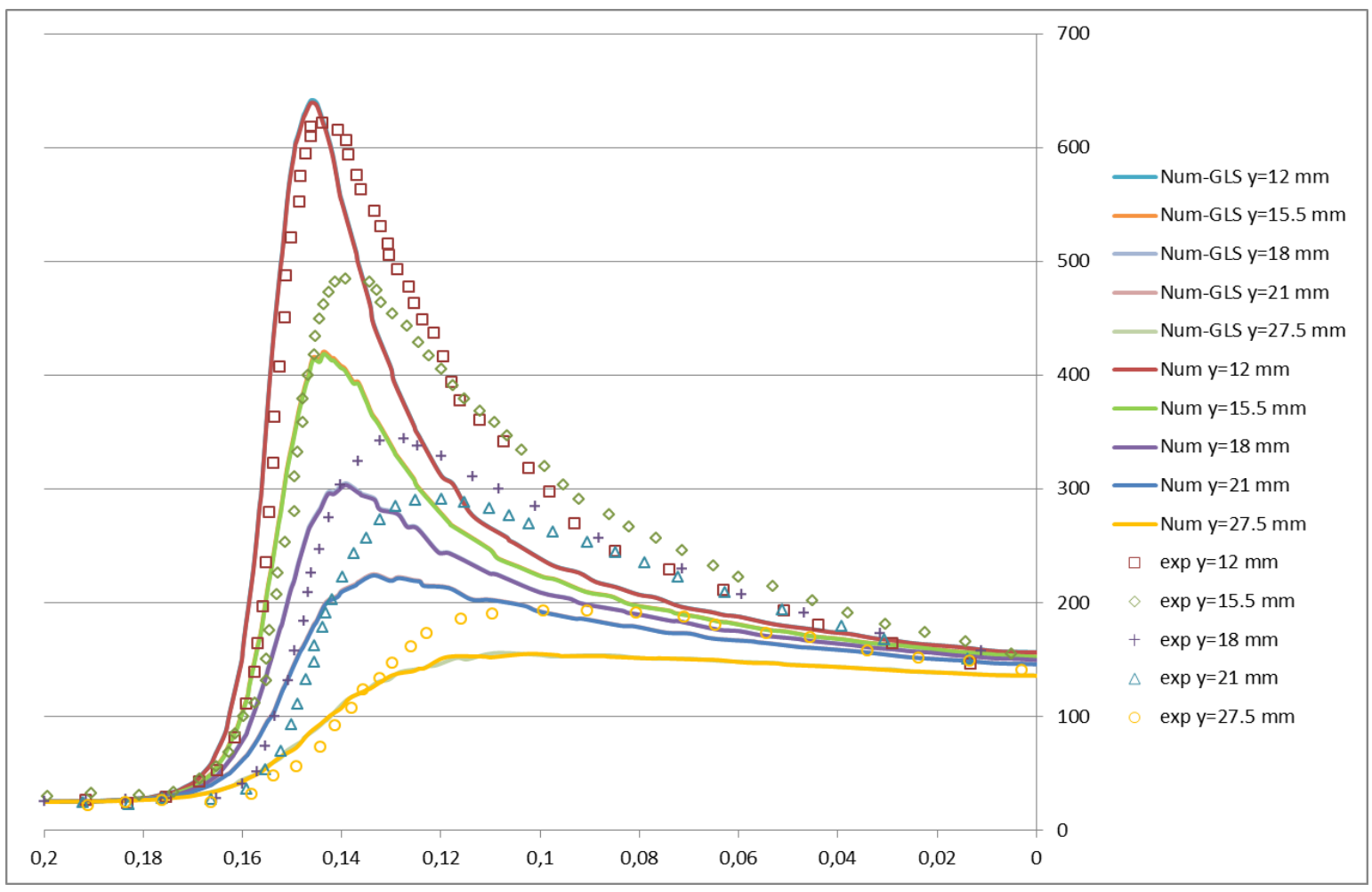

Figure 15. Temperatures at the end of the simulation, along four different lines parallel to the welding line on the top surface of the work-pieces. Comparison between the experimental results (symbols) reported by Zhu and Chao [83] and the numerical results (lines), using ASGS (GLS) and OSGS stabilization methods.

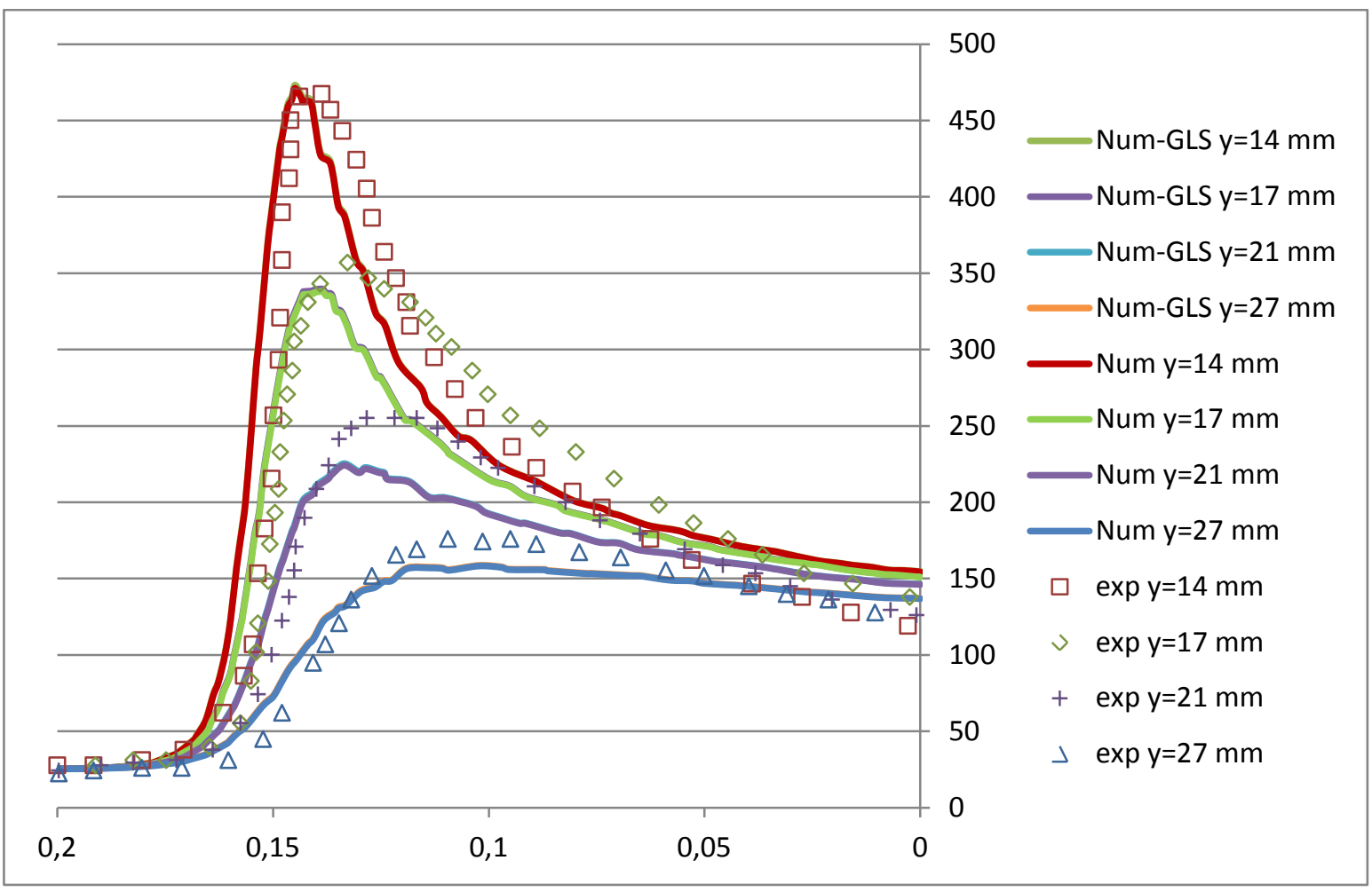

Figure 16. Temperatures at the end of the simulation, along four different lines parallel to the welding line on the bottom surface of the work-pieces. Comparison between the experimental results (symbols) reported by Zhu and Chao [83] and the numerical results (lines), using ASGS

(GLS) and OSGS stabilization methods. 
Figures 15 and 16 show a comparison between the experimental results reported by Zhu and Chao [83] and the numerical results, using ASGS (GLS) and OSGS stabilization methods, obtained for the temperature along five different lines parallel to the welding line on the top surface $(\mathrm{z}=3.18 \mathrm{~mm})$ and four different lines parallel to the welding line on the bottom surface $(\mathrm{z}=0.0 \mathrm{~mm})$ of the work-pieces, respectively.

The five lines considered on the top of the surface $(z=3.18 \mathrm{~mm})$ of the work-pieces are located at $y=12 \mathrm{~mm}, y=15.5 \mathrm{~mm}, \mathrm{y}=18 \mathrm{~mm}, \mathrm{y}=21 \mathrm{~mm}$ and $\mathrm{y}=27.5 \mathrm{~mm}$ of the welding line. As it is shown in Figure 5, numerical results obtained compare qualitatively well with the experimental ones. The peak temperature for the lines located at $y=12 \mathrm{~mm}$, the one which is closest to the welding line, is remarkably well caught by the numerical simulation, both in position and value. Peak temperatures for lines located at $y=15.5 \mathrm{~mm}, \mathrm{y}=18 \mathrm{~mm}$ and $\mathrm{y}=21 \mathrm{~mm}$ are slightly underestimated by the numerical simulation, but the values at $\mathrm{x}=0$ fit well with the experimental results. On the other hand, ASGS (GLS) and OSGS stabilization methods yield basically the same results.

The four lines considered on the bottom surface $(\mathrm{z}=0.0 \mathrm{~mm})$ of the work-pieces are located at $y=14 \mathrm{~mm}, y=17 \mathrm{~mm}, y=21 \mathrm{~mm}$ and $y=27 \mathrm{~mm}$ of the welding line. As it is shown in Figure 6, numerical results obtained compare qualitatively well with the experimental ones. Once again, the peak temperature for the line located at $\mathrm{y}=14 \mathrm{~mm}$, the one which is closest to the welding line, is caught remarkably well by the numerical simulation, both in position and value. Similarly, peak temperatures for lines located at $y=17 \mathrm{~mm}$ and $\mathrm{y}=27 \mathrm{~mm}$ are also quite remarkably well caught, both in position and value, while the peak temperature for the line located at $\mathrm{y}=21 \mathrm{~mm}$ is slightly underestimated by the numerical simulation. Temperatures at $\mathrm{x}=0$ fit pretty well with the experimental results. ASGS (GLS) and OSGS stabilization methods yield virtually identical results.

\section{Conclusions}

In this paper, different aspects related to the computational modeling and the sub-grid scale finite element stabilization of incompressibility and convection in the numerical simulation of friction stir welding processes have been addressed. Two suitable rigid-thermoplastic constitutive models, Norton-Hoff and Sheppard-Wright, have been introduced. Within the paradigmatic framework of the multiscale methods, suitable ASGS and OSGS stabilization methods have been introduced for a fully incompressible, quasi-static, transient coupled thermomechanical formulation, using an Eulerian description. Classical GLS and SUPG stabilization methods can be recovered as a particular case of the sub-grid scale stabilization framework developed. An assessment of the sub-grid scale finite element stabilization methods has been performed. Two numerical examples have been considered. The first one deals with numerical simulation of a coupled thermomechanical flow in a 2D rectangular domain. Steadystate and transient conditions have been considered. Using the GLS method to stabilize the pressure, temperature distributions using SUPG and OSGS convection stabilization methods have been compared. Numerical results show that OSGS stabilization method yields slightly better temperature results. On the other hand, using the SUPG method to stabilize the convective term of the temperature equation, pressure and velocity contours using GLS and 
OSGS pressure stabilization methods have been also compared. Numerical results show that OSGS stabilization method yield better results, particularly at the inflow and outflow sections. Finally, the second example shows a 3D numerical simulation of a FSW process. ASGS (GLS and SUPG) and OSGS stabilization methods have been used. Numerical results have been compared with experimental ones available. A good agreement on the temperature distribution along different lines parallel to the welding line on the bottom and top surfaces of the workpieces has been obtained and predicted peak temperatures compare well, both in value and position, with the experimental results available.

\section{Acknowledgements}

This work has been supported by the European Commission under the STREP project of the VI Framework Programme "Detailed Multi-Physics Modeling of Frictional Stir Welding" (DEEPWELD), the European Research Council under the Advanced Grant: ERC-2009-AdG "Real Time Computational Mechanics Techniques for Multi-Fluid Problems", the Spanish Ministerio de Educación y Ciencia under the PROFIT project CIT-020400-2007-82: "Nuevas Herramientas para Optimizar el Proceso de Soldadura por Fricción" (FSWNET) and the project of the Plan Nacional de I+D+I (2004-2007) "Simulación Numérica del Proceso de Soldadura Mediante Batido por Fricción" (FSW).

\section{References}

1. C. Agelet de Saracibar, Numerical analysis of coupled thermomechanical contact problems. Computational model and applications, Archives of Computational Methods in Mechanics 5 (1998) 243-301. DOI: 10.1007/BF02897875.

2. C. Agelet de Saracibar, M. Cervera, M. Chiumenti, On the formulation of coupled thermoplastic problems with phase-change, International Journal of Plasticity 15 (1999) 134. DOI: 10.1016/S0749-6419(98)00055-2.

3. C. Agelet de Saracibar, M. Cervera, M. Chiumenti, On the constitutive modeling of coupled thermomechanical phase-change problems, International Journal of Plasticity 17 (2001) 1565-1622. DOI: 10.1016/S0749-6419(00)00094-2.

4. C. Agelet de Saracibar, M. Chiumenti, Q. Valverde, M. Cervera, On the orthogonal sub-grid scale pressure stabilization of small and finite deformation J2 plasticity, in C. Agelet de Saracibar (ed), Monograph Series on Computational Methods in Forming Processes, Monograph no. 2, CIMNE, Barcelona, Spain, 2004.

5. C. Agelet de Saracibar, M. Chiumenti, Q. Valverde, M. Cervera, On the orthogonal sub-grid scale pressure stabilization of finite deformation J2 plasticity, Comput. Methods Appl. Mech. Engrg. 195 (2006) 1224-1251. DOI: 10.1016/j.cma.2005.04.007.

6. C. Agelet de Saracibar, M. Chiumenti, D. Santiago, N. Dialami, G. Lombera, On the numerical modeling of FSW processes, Proceedings of the International Symposium on Plasticity and its Current Applications, Plasticity 2010, St. Kitts, St. Kitts and Nevis, January 3-8, 2010.

7. C. Agelet de Saracibar, M. Chiumenti, D. Santiago, M. Cervera, N. Dialami, G. Lombera, A computational model for the numerical simulation of FSW processes, in F. Barlat, Y.H. Moon, M.G. Lee (eds), NUMIFORM 2010: Proceedings of the 10th International Conference on Numerical Methods in Industrial Forming Processes, Dedicated to Professor 
O. C. Zienkiewicz (1921-2009), Pohang, South Korea, 13-17 June 2010, AIP Conference Proceedings, Vol. 1252, 2010, pp. 81-88. DOI: 10.1063/1.3457640.

8. C. Agelet de Saracibar, M. Chiumenti, M. Cervera, N. Dialami, D. Santiago, G. Lombera, Advances in the numerical simulation of 3D FSW processes, Proceedings of the International Symposium on Plasticity and its Current Applications, Plasticity 2011, Puerto Vallarta, Mexico, January 3-8, 2011.

9. C. Agelet de Saracibar, R. López, B. Ducoeur, M. Chiumenti, B. de Meester, Un modelo numérico para la simulación de disolución de precipitados en aleaciones de aluminio con endurecimiento utilizando redes neuronales, Revista Internacional de Métodos Numéricos para Cálculo y Diseño en la Ingeniería 2013; 29(1): 29-37. DOI: 10.1016/j.rimni.2012.02. 003.

10. F. Armero, J.C. Simo, A new unconditionally stable fractional step method for non-linear coupled thermomechanical problems, International Journal for Numerical Methods in Engineering 35 (1991) 737-766. DOI: 10.1002/nme.1620350408.

11. F. Armero, J.C. Simo, Product formula algorithms for nonlinear coupled thermo-plasticity: Formulation and non-linear stability analysis, SUDAM Report \#92-4, Department of Mechanical Engineering, Division of Applied Mechanics, Stanford University, Palo Alto, CA, USA, 1992.

12. F. Armero, J.C. Simo, A priori stability estimates and unconditionally stable product formula algorithms for non-linear coupled thermoplasticity, International Journal of Plasticity 9, 6 (2003) 749-782

13. A. Askari, S. Silling, B. London, M. Mahoney, Modeling and analysis of friction stir welding processes, Proceedings of the $4^{\text {th }}$ International Symposium on Friction Stir Welding (4ISFSW), GKSS Workshop, Park City, Utah, USA, May 14-16, 2003.

14. M. Avila, J. Principe, R. Codina, Finite element dynamical sub-grid scale approximation of low Mach number flow equations, in E. Dvorkin, M. Goldschmit, M. Storti (eds), Proceedings of the Asociación Argentina de Mecánica Computacional, Buenos Aires, Argentina, 15-18 Noviembre 2010, Mecánica Computacional, Vol XXIX, 7967-7983, 2010.

15. G. Bendzsak, T. North, C. Smith, An experimentally validated 3D model for friction stir welding, Proceedings of the $2^{\text {nd }}$ International Symposium on Friction Stir Welding (2ISFSW), Gothenburg, Sweden, June 27-29, 2000.

16. G. Bendzsak, T. North, C. Smith, Material properties relevant to 3-D FSW modeling, Proceedings of the $2^{\text {nd }}$ International Symposium on Friction Stir Welding (2ISFSW), Gothenburg, Sweden, June 27-29, 2000.

17. F. Brezzi, M. Fortin, Mixed and hybrid finite element methods, Springer Series in Computational Mathematics, vol. 15, Springer-Verlag, New York, 1991.

18. G. Buffa, J. Hua, R. Shivpuri, L. Fratini, A continuum-based fem model for friction stir welding - model development, Materials Science and Engineering A 419 (2006) 389-396. DOI: 10.1016/j.msea.2005.09.040.

19. P. Bussetta, N. Dialami, R. Boman, M. Chiumenti, C. Agelet de Saracibar, M. Cervera, J.P. Ponthot, Comparison of a fluid and a solid approach for the numerical simulation of friction stir welding with a non-cylindrical pin, Proceedings of the International Conference on Coupled Problems in Engineering 2013, Santa Eulalia, Ibiza, Spain, June 17-19, 2013.

20. P. Bussetta, N. Dialami, R. Boman, M. Chiumenti, C. Agelet de Saracibar, M. Cervera, J.P. Ponthot, Numerical simulation of friction stir welding process with different pin geometries based on a combined ALE/remeshing formulation, Steel Research International 2013 (submitted). 
21. M. Cervera, C. Agelet de Saracibar, M. Chiumenti, COMET - A Coupled Mechanical and Thermal Analysis Code. Data Input Manual. Version 5.0, Technical Report IT-308, CIMNE, Barcelona, Spain, 2002. http://www.cimne.com/comet.

22. M. Cervera, M. Chiumenti, Q. Valverde, C. Agelet de Saracibar, Mixed linear/linear simplicial elements for incompressible elasticity and plasticity, Comput. Methods Appl. Mech. Engrg. 192 (2003) 5249-5263. DOI: 10.1016/j.cma.2003.07.007.

23. M. Cervera, M. Chiumenti, C. Agelet de Saracibar, Shear band localization via local J2 continuum damage mechanics, Comput. Methods. Appl. Mech. Engrg. 193 (2004) 849-880. DOI: $10.1016 /$ j.cma.2003.11.009.

24. M. Cervera, M. Chiumenti, C. Agelet de Saracibar, Softening, localization and stabilization: capture of discontinuous solutions in J2 plasticity, International Journal for Numerical and Analytical Methods in Geomechanics 2004; 28:373-393. DOI: 10.1002/nag.341.

25. M. Cervera, M. Chiumenti, R. Codina, Mixed stabilized finite element methods in nonlinear solid mechanics. Part I: Formulation, Comput. Methods. Appl. Mech. Engrg. 199 (2010) 2559-2570. DOI: 10.1016/j.cma.2010.04.006.

26. M. Cervera, M. Chiumenti, R. Codina, Mixed stabilized finite element methods in nonlinear solid mechanics. Part II: Strain localization, Comput. Methods. Appl. Mech. Engrg. 199 (2010) 2571-2589. DOI: 10.1016/j.cma.2010.04.005.

27. M. Cervera, M. Chiumenti, R. Codina, Mesh objective modeling of cracks using continuous linear strain and displacement interpolations, International Journal for Numerical Methods in Engineering 2011; 87 : 962-987. DOI: 10.1002/nme.3148.

28. C.M. Chen, R. Kovacevic, Finite element modeling of friction stir welding - thermal and thermomechanical analysis, Int. J. Machine Tools and Manufacture 43 (2003) 1319-1326. DOI: 10.1016/S0890-6955(03)00158-5.

29. M. Chiumenti, Q. Valverde, C. Agelet de Saracibar, M. Cervera, A stabilized formulation for incompressible elasticity using linear displacement and pressure interpolations, Comput. Methods Appl. Mech. Engrg. 191 (2002) 5253-5264. DOI: 10.1016/S0045-7825(02)004437.

30. M. Chiumenti, Q. Valverde, C. Agelet de Saracibar, M. Cervera, A stabilized formulation for incompressible plasticity using linear triangles and tetrahedra, International Journal of Plasticity 20 (2004) 1487-1504. DOI: 10.1016/j.ijplas.2003.11.009.

31. M. Chiumenti, M. Cervera, A. Salmi, C. Agelet de Saracibar, N. Dialami, K. Matsui, Finite element modeling of multi-pass welding and shaped metal deposition processes, Comput. Methods. Appl. Mech. Engrg. 199 (2010) 2343-2359. DOI: 10.1016/j.cma.2010.02.018.

32. M. Chiumenti, M. Cervera, C. Agelet de Saracibar, A mixed stabilized finite element formulation for strain localization analysis, Proceedings of the $11^{\text {th }}$ Pan-American Congress of Applied Mechanics - PACAM XI, January 4-8, 2010, Foz do Iguaçu, PR, Brazil.

33. M. Chiumenti, M. Cervera, C. Agelet de Saracibar, N. Dialami, Numerical modelling of friction stir welding processes, Comput. Methods. Appl. Mech. Engrg. 254 (2013) 353-369. DOI: $10.1016 /$ j.cma.2012.09.013.

34. M. Chiumenti, M. Cervera, C. Agelet de Saracibar, N. Dialami, A novel stress-accurate FE technology for highly non-linear analysis with incompressibility constraint. Application to the numerical simulation of the FSW process, AIP Conference Proceedings, Proceedings of the International Conference on Numerical Methods in Forming Processes, NUMIFORM 2013, Shenyang, China, 2013 (to be published).

35. D. Christ, M. Cervera, M. Chiumenti, C. Agelet de Saracibar, A mixed finite element formulation for incompressibility using linear displacement and pressure interpolations, Monograph no. 77, CIMNE, Barcelona, Spain, 2003. 
36. R. Codina, J. Blasco, A finite element formulation for the Stokes problem allowing equal velocity-pressure interpolation, Comput. Methods Appl. Mech. Engrg. 143 (1997) 373-391. DOI: $10.1016 / \mathrm{S} 0045-7825(96) 01154-1$.

37. R. Codina, Comparison of some finite element methods for solving the diffusionconvection-reaction equations, Comput. Methods Applied Mech. and Engrg. 156 (1998) 185-210. DOI: 10.1016/S0045-7825(97)00206-5.

38. R. Codina, J. Blasco, Stabilized finite element method for transient Navier-Stokes equations based on pressure gradient projection, Comput. Methods Appl. Mech. Engrg. 182 (2000) 287-300. DOI: 10.1016/S0045-7825(99)00194-2.

39. R. Codina, Stabilization of incompressibility and convection through orthogonal sub-scales in finite element methods, Comput. Methods Appl. Mech. Engrg. 190 (2000) 1579-1599. DOI: $10.1016 / \mathrm{S} 0045-7825(00) 00254-1$.

40. R. Codina, J. Blasco, Analysis of a pressure-stabilized finite element approximation of the stationary Navier-Stokes equations, Numer. Math. 87 (2000) 59-81. DOI: $10.1007 / \mathrm{s} 002110000174$.

41. R. Codina, A stabilized finite element method for generalized stationary incompressible flows, Comput. Methods Appl. Mech. Engrg. 190 (2001) 2681-706. DOI: 10.1016/S00457825(00)00260-7.

42. R. Codina, Stabilized finite element approximation of transient incompressible flows using orthogonal subscales, Comput. Methods Appl. Mech. Engrg. 191 (2002) 4295-4321. DOI: 10.1016/S0045-7825(02)00337-7.

43. R. Codina, J. Principe, O. Guasch, S. Badia, Time dependent subscales in the stabilized finite element approximation of incompressible flow problems, Comput. Methods Appl. Mech. Engrg. 196 (2007) 2413-2430. DOI: 10.1016/j.cma.2007.01.002.

44. R. Codina, J. Principe, Dynamic subscales in the finite element approximation of thermally coupled incompressible flows, International Journal for Numerical Methods in Fluids 54 (2007) 707-730. DOI: 10.1002/fld.1481.

45. R. Codina, J. Principe, M. Avila, Finite element approximation of turbulent thermally coupled incompressible flows with numerical sub-grid scale modelling, International Journal of Numerical Methods for Heat and Fluid Flow 20(5):492-516, 2010. DOI: 10.1108/09615531011048213.

46. P. Colegrove, M. Painter, D. Graham, T. Miller, Three dimensional flow and thermal modelling of the friction stir welding process, Proceedings of the $2^{\text {nd }}$ International Symposium on Friction Stir Welding (2ISFSW), Gothenburg, Sweden, June 27-29, 2000.

47. P. Colegrove, H. Shercliff, P. Threadgill, Modelling the friction stir welding of aerospace alloys, Proceedings of the $5^{\text {th }}$ International Symposium on Friction Stir Welding (5ISFSW), Metz, France, September 14-16, 2004.

48. T. De Vuyst, L. D'Alvise, A. Simar, B. de Meester, S. Pierret, Finite element modelling of friction stir welding aluminium alloys plates - Inverse analysis using a genetic algorithm, Welding in the World, 49, 3/4, 44-55, 2005.

49. T. De Vuyst, L. D'Alvise, A. Simar, B. de Meester, S. Pierret, Inverse analysis using a genetic algorithm for the finite element modelling of friction stir welding, Proceedings of the $5^{\text {th }}$ International Symposium on Friction Stir Welding (5ISFSW), Metz, France, September 14-16, 2004.

50. T. De Vuyst, L. D'Alvise, A. Robineau, J.C. Goussain, Material flow around a friction stir welding tool - Experiment and Simulation, Proceedings of the 8th International Seminar on Numerical Analysis of Weldability, Graz, Austria, September 25-27, 2006.

51. T. De Vuyst, L. D'Alvise, A. Robineau, J.C. Goussain, Simulation of the material flow around a friction stir welding tool, Proceedings of the 6th International Symposium on Friction Stir Welding (6ISFSW), Saint-Sauveur, Quebec, Canada, October 10-13, 2006. 
52. N. Dialami, M. Chiumenti, M. Cervera, C. Agelet de Saracibar, An apropos kinematic framework for the numerical modelling of Friction Stir Welding, Computers and Structures 117 (2013) 48-57. DOI: 10.1016/j.compstruc.2012.12.006.

53. P. Dong, F. Lu, J.K. Hong, Z. Cao, Coupled thermomechanical analysis of friction stir welding process using simplified models, Sci. Technol. Weld. Joining, 2001, 6(5), 281-287. DOI: $10.1179 / 136217101101538884$.

54. GiD: The Personal Pre and Post processor, CIMNE, 2011. http://www.gidhome.com.

55. P. Heurtier, C. Desrayaud, F. Montheillet, A thermomechanical analysis of the friction stir process, Materials Science Forum 396 (2002) 1537-1542. DOI: 10.4028/www.scientific. net/MSF.396-402.1537.

56. P. Heurtier, M.J. Jones, C. Desrayaud, J.H. Driver, F. Montheillet, D. Allehaux, Mechanical and thermal modeling of friction stir welding, Journal of Materials Processing Technology 171 (2006) 348-357. DOI: 10.1016/j.jmatprotec.2005.07.014.

57. T.J.R. Hughes, L. Franca, M. Balestra, A new finite element formulation for computational fluid dynamics: V. Circumventing the Babuska-Brezzi condition: A stable Petrov-Galerkin formulation of the Stokes problem accommodating equal order interpolations, Comput. Methods Appl. Mech. Engrg. 59 (1986) 85-99. DOI: 10.1016/0045-7825(86)90025-3.

58. T.J.R. Hughes, Multiscale phenomena: Green's function, the Dirichlet-to-Neumann formulation, subgrid scale models, bubbles and the origins of stabilized formulations, Comput. Methods Appl. Mech. Engrg. 127 (1995) 387-401. DOI: 10.1016/00457825(95)00844-9.

59. T.J.R. Hughes, G.R. Feijóo, L. Mazzei, J.B. Quincy, The variational multiscale method - a paradigm for computational mechanics, Comput. Methods Appl. Mech. Engrg. 166 (1998) 3-24. DOI: 10.1016/S0045-7825(98)00079-6.

60. T.J.R. Hughes, G. Scovazzi, L. Franca, Multiscale and stabilized methods, in E. Stein, R. de Borst, T.J.R. Hughes (eds), Encyclopedia of Computational Mechanics, Wiley, Chichester, 2004. DOI: $10.1002 / 0470091355$.

61. A.M. Jorge Jr., O. Balancin, Prediction of steel flow stresses under hot working conditions, Materials Research, Vol. 8, No. 3, 309-315, 2005. DOI: 10.1590/S1516-143920050003000 15.

62. M. Khandkar, J. Khan, Thermal modeling of overlap friction stir welding for Al-alloys, Journal of Materials Processing and Manufacturing Science 10 (2001) 91-105. DOI: $10.1177 / 1062065602010002613$.

63. M. Khandkar, J. Khan, A. Reynolds, Prediction of temperature distribution and thermal history during friction stir welding: input torque based model, Science and Technology of Welding and Joining 8, 3 (2003) 165-174. DOI: 10.1179/136217103225010943.

64. M. Langerman, E. Kvalvik, Modeling plasticized aluminum flow and temperature fields during friction stir welding, Proceedings of the $6^{\text {th }}$ ASME-JSME Thermal Engineering Joint Conference, Hapuna Beach Prince Hotel, Kohala Coast, Hawaii Island, Hawaii, USA, March 16-20, 2003.

65. R. López, B. Ducoeur, M. Chiumenti, B. de Meester, C. Agelet de Saracibar, Modeling precipitate dissolution in hardened aluminium alloys using neural networks, International Journal of Material Forming, Vol. 1, Supl. 1, (2008) 1291-1294, DOI: 10.1007/s12289-0080139-4.

66. J.C. McClure, W. Tang, L.E. Murr, X. Guo, Z. Feng, J.E. Gould, A thermal model of friction stir welding, Proceedings of the $5^{\text {th }}$ International Conference on Trends in Welding Research, Pine Mountain, Georgia, USA, June 1-5, 1998, pp. 590-595.

67. N. Nikiforakis, Towards a whole system simulation of FSW, Proceedings of the 2nd FSW Modelling and Flow Visualisation Seminar, GKSS Forschungszentrum, Geesthacht, Germany, January 31-February 1, 2005. 
68. J. Principe, Subgrid scale stabilized finite elements for low speed flows, Ph.D. Thesis, Technical University of Catalonia, Barcelona, Spain, 2008.

69. J. Principe, R. Codina, A stabilized finite element approximation of low speed thermally coupled flows, International Journal of Numerical Methods for Heat and Fluid Flow 18(7/8):835-867, 2008. DOI: 10.1108/09615530810898980.

70. J. Principe, R. Codina, Mathematical models for thermally coupled low speed flows. Advances in Theoretical and Applied Mechanics 2 (2009) 93-112. http://www.mhikari.com/atam/atam2009/atam1-4-2009/principeATAM1-4-2009.pdf.

71. D. Santiago, G. Lombera, S. Urquiza, C. Agelet de Saracibar, M. Chiumenti, Modelado termo-mecánico del proceso de Friction Stir Welding utilizando la geometría real de la herramienta, Revista Internacional de Métodos Numéricos para Cálculo y Diseño en Ingeniería 26 (2010) 293-303. DOI: 10.1016/j.rimni.2012.02.003.

72. H. Schmidt, J. Hattel, Modelling thermo mechanical conditions at the tool/matrix interface in Friction Stir welding, Proceedings of the $5^{\text {th }}$ International Symposium on Friction Stir Welding (5ISFSW), Metz, France, September 14-16, 2004.

73. T.U. Seidel, A.P. Reynolds, Two-dimensional friction stir welding process model based on fluid mechanics, Science and Technology of Welding and Joining 8, 3 (2003) 175-183. DOI: $10.1179 / 136217103225010952$.

74. H.R. Shercliff, M.J. Russell, A. Taylor, T.L. Dickerson, Microstructural modeling in friction stir welding of 2000 series aluminium alloys, Mécanique \& Industries 6 (2005) 2535. DOI: $10.1051 /$ meca:2005004.

75. Q. Shi, T. Dickerson, H. Shercliff, Thermo-mechanical FE modeling of friction stir welding of AL-2024 including tool loads, Proceedings of the $4^{\text {th }}$ International Symposium on Friction Stir Welding (4ISFSW), Park City, Utah, USA, May 14-16, 2003.

76. M. Song, R. Kovacevic, Numerical and experimental study of the heat transfer process in friction stir welding, Journal of Engineering Manufacture 217, Part B (2003) 73-85.

77. W.M. Thomas, E.D. Nicholas, J.C. Needham, M.G. Murch, P. Temple-Smith, C.J. Dawes, Friction stir butt welding. GB Patent No. 9125978.8, International Patent No. PCT/GB92/02203, 1991.

78. P. Ulysse, Three-dimensional modeling of the friction stir-welding process, International Journal of Machine Tools and Manufacture 42 (2002) 1549-1557. DOI: 10.1016/S08906955(02)00114-1.

79. S. Xu, X. Deng, A.P. Reynolds, T.U. Seidel, Finite element simulation of material flow in friction stir welding, Science and Technology of Welding and Joining 6 (3) 191-193, 2001. DOI: $10.1179 / 136217101101538640$.

80. S. Xu, X. Deng, Two and three-dimensional finite element models for the friction stir welding process, Proceedings of the $4^{\text {th }}$ International Symposium on Friction Stir Welding (4ISFSW), Park City, Utah, USA, May 14-16, 2003.

81. S. Xu, X. Deng, Two and three-dimensional finite element models for the friction stir welding process, University of South Carolina, Department of Mechanical Engineering, Columbia, South Carolina 29208, USA, 2004.

82. H. Zhao, Friction Stir Welding (FSW) simulation using an Arbitrary Lagrangian-Eulerian (ALE) moving mesh approach, Ph.D. Dissertation, West Virginia University, Morgantown, West Virginia, USA, 2005. http://hdl.handle.net/10450/4367.

83. X.K. Zhu, Y.J. Chao, Numerical simulation of transient temperature and residual stresses in friction stir welding of 304L stainless steel, Journal of Materials Processing Technology 146, 2 (2004) 263-272. DOI: 10.1016/j.jmatprotec.2003.10.025. 